Universidade de SÃo Paulo

Faculdade de Economia, Administração e Contabilidade de Ribeirão Preto Departamento DE ECONOMia

Programa de Pós-Graduação em Economia - Área: Economia Aplicada

Renata Tavanielli

\title{
Modelo de taxa de juros a termo com mudanças de regime
}

Orientador: Prof. Dr. Márcio Poletti Laurini

Ribeirão Preto

2019 


\section{Prof. Dr. Vahan Agopyan \\ Reitor da Universidade de São Paulo}

Prof. Dr. André Lucirton Costa

Diretor da Faculdade de Economia, Administração e Contabilidade de Ribeirão Preto

Prof. Dr. Sérgio Kannebley Júnior

Chefe do Departamento de Economia

Prof. Dr. Sérgio Naruhiko Sakurai

Coordenador do Programa de Pós-graduação em Economia - Área:

Economia Aplicada 
Renata Tavanielli

\section{Modelo de taxa de juros a termo com mudanças de regime}

Dissertação apresentada ao Programa de Pós-Graduação em Economia - Área: Economia Aplicada da Faculdade de Economia, Administração e Contabilidade de Ribeirão Preto da Universidade de São Paulo, para obtenção de título de Mestre em Ciências. Versão Corrigida. A original encontra-se disponível na FEA-RP/USP.

ORIENTADOR: Prof. Dr. Márcio Poletti Laurini

Ribeirão Preto 
Autorizo a reprodução e divulgação total ou parcial deste trabalho, por qualquer meio convencional ou eletrônico, para fins de estudo e pesquisa, desde que citada a fonte.

Tavanielli, Renata.

Modelo de taxa de juros a termo com mudanças de regime. Ribeirão Preto, 2019.

68 p.: il; $30 \mathrm{~cm}$

Dissertação de Mestrado, apresentada à Faculdade de Economia, Administração e Contabilidade de Ribeirão Preto/USP. Área de concentração: Economia Aplicada.

Orientador: Laurini, Márcio Poletti.

1. Estrutura a Termo. 2. Mudanças de Regime. 3. MCMC.

O presente trabalho foi realizado com apoio da Coordenação de Aperfeiçoamento de Pessoal de Nível Superior - Brasil (CAPES) - Código de Financiamento 001. 


\section{Agradecimentos}

Agradeço ao meu orientador pelo constante apoio à pesquisa e ao incentivo à busca pelo conhecimento. A todas as suas disciplinas, que foram muito importantes para minha formação, e por proporcionar toda a estrutura física necessária para as estimações deste trabalho.

Aos professores, Andreza Aparecida Palma, João Frois Caldeira e Sérgio Kannebley Jr., pelas contribuições a este trabalho na banca de defesa, e aos professores, Alex Luiz Ferreira e Fábio Augusto Reis Gomes, nas bancas de qualificação e pré-defesa. A todos os professores do PPGE pela busca constante pela melhor formação de seus alunos.

À USP como um todo por toda minha formação superior e ao financiamento da Capes e da Fundace ao longo do mestrado.

À minha família, em especial à minha mãe, por tudo. 



\section{Resumo}

Tavanielli, R. Modelo de taxa de juros a termo com mudanças de regime. Dissertação (Mestrado) - Faculdade de Economia, Administração e Contabilidade de Ribeirão Preto, Universidade de São Paulo, Ribeirão Preto, 2019.

São propostas diversas extensões do modelo dinâmico de Nelson-Siegel para a análise do ajuste e da acurácia preditiva utilizando dados de DI do mercado brasileiro, incorporando, além de variáveis macroeconômicas, a possibilidade de mudanças de regime nos parâmetros dos modelos. As abordagens utilizadas são motivadas por evidências que sugerem mudanças de regime na curva de juros dos EUA, que é mais bem comportada que as de países em desenvolvimento, e evidências de mudança de regime em variáveis macroeconômicas brasileiras. Para a estimação verificamos que, além da incorporação de variáveis macroeconômicas, modelos com maior flexibilidade apresentam melhor ajuste. Na previsão fora da amostra, o desempenho dos modelos dependem do horizonte de previsão e da maturidade considerada, sendo que pelo procedimento do Model Confidence Set os modelos com mudança de regime se destacam. O modelo com mudança de regime nas variáveis latentes e nos fatores macroeconômicos (MDNS-MMacroEnd) destaca-se para maturidades mais elevadas para o horizonte de previsão de 1 mês. Para o horizonte de previsão de 12 meses, o modelo com mudança de regime baseado no artigo de So et al. (1998) nas variáveis macroeconômicas (MDNS-Smacro) apresenta melhor poder preditivo na maioria das maturidades analisadas. Já para o horizonte de 60 meses, o modelo com mudança de regime no fator de decaimento (MDNS- $\lambda$ ) possui melhor acurácia preditiva para a maioria das maturidades.

Palavras-chave: Estrutura a Termo, Mudanças de Regime, MCMC. 



\begin{abstract}
Tavanielli, R. Yield curve model with regime switches. Dissertation (Master Degree) - School of Economics, Business and Accounting at Ribeirão Preto, University of São Paulo, Ribeirão Preto, 2019.

This thesis estimates versions of the dynamic Nelson-Siegel model, with macro factors and different methods of regime switch, for Brazilian ID data to analyze the fit and forecast accuracy. Our modeling approach is motivated by evidences suggesting breaks in the USA yield curve, which is more well behave than curves in developing countries, and evidences of regime switch in Brazilian macroeconomics variables. For the fit, the models with macro factors and more flexibility outperform the others. For out of sample forecast, the performance of the models depends of the forecast horizon and the maturity, in which by the procedure of the Model Confidence Set, models with regime switch stand out. Model with regime switch in the latent and macro factors (MDNS-MMacroEnd) outperforms in long maturities at shortest forecast horizon. For twelve month horizon, the model with regime switch based on So et al. (1998) in the macro factors (MDNS-Smacro) outperforms the others in most of the maturities. However, for the sixty month horizon, model with the regime switch in the decay factor $(\operatorname{MDNS}-\lambda)$ has better predictive accuracy for most of the maturities.
\end{abstract}

Keywords: Yield Curve, Regime Switch, MCMC. 

Sumário

Lista de ilustrações . . . . . . . . . . . . . . . . . . 10

Lista de tabelas . . . . . . . . . . . . . . . . . . 11

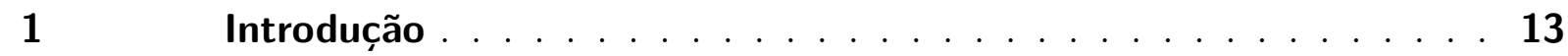

2 Referencial Bibliográfico . . . . . . . . . . . . . . 17

3 Metodologia . . . . . . . . . . . . . . . 29

$4 \quad$ Base de Dados . . . . . . . . . . . . . . . . . . 33

$5 \quad$ Resultados . . . . . . . . . . . . . . . . . 37

5.1 Previsão Fora da Amostra . . . . . . . . . . . . . . . . . . 54

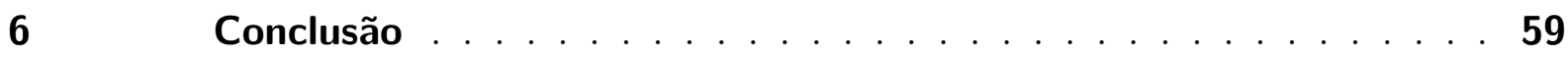

REFERÊNCIAS .................... 61

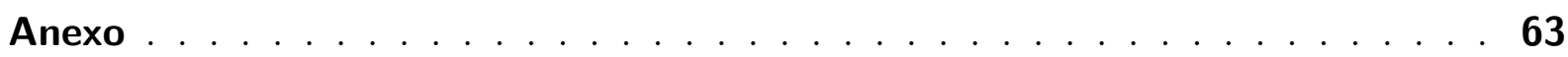




\section{Lista de ilustrações}

Figura 1 - Gráfico dos Dados Observados de DI para as 17 diferentes Maturidades 34

Figura 2 - Gráfico da Mediana para cada uma das Maturidades . . . . . . . . . . 35

Figura 3 - Gráfico Dados Macroeconômicos . . . . . . . . . . . . . 35

Figura 4 - Gráfico Variáveis Latentes MDNS . . . . . . . . . . . . . . . . 38

Figura 5 - Gráfico Variáveis Latentes MDNS-M . . . . . . . . . . . . . . . . . 39

Figura 6 - Gráfico Variáveis Latentes MDNS-P . . . . . . . . . . . . . . . . 40

Figura 7 - Gráfico Variáveis Latentes MDNS- $\lambda \ldots \ldots$. . . . . . . . . . . . 41

Figura 8 - Gráfico Variáveis Latentes MDNS-Macro . . . . . . . . . . . . . . . . 42

Figura 9 - Gráfico Variáveis Latentes MDNS-MMacro . . . . . . . . . . . . . . . . 43

Figura 10 - Gráfico Variáveis Latentes MDNS-PMacro . . . . . . . . . . . . . . . . 44

Figura 11 - Gráfico Variáveis Latentes MDNS-S . . . . . . . . . . . . . . . . . . 45

Figura 12 - Gráfico Variáveis Latentes MDNS-MMacroEnd . . . . . . . . . . . . . 46

Figura 13 - Gráfico Variáveis Latentes MDNS-PMacroEnd . . . . . . . . . . . . . . 47

Figura 14 - Gráfico Comparativo Variáveis Latentes MDNS e MDNS-S $\lambda$. . . . . . 48

Figura 15 - Gráfico Comparativo Variáveis Latentes MDNS e MDNS-SmediaMacro 49

Figura 16 - Gráfico Comparativo Variáveis Latentes MDNS e MDNS-Smacro . . . 50

Figura 17 - Gráfico Comparativo das Variáveis Latentes . . . . . . . . . . . . . 52

Figura 18 - Model Confidence Set - Horizonte de Previsão 1 mês* . . . . . . . . . . 63

Figura 19 - Model Confidence Set - Horizonte de Previsão 12 meses* . . . . . . . . 65

Figura 20 - Model Confidence Set - Horizonte de Previsão 60 meses* . . . . . . . . 67 


\section{Lista de tabelas}

Tabela 1 - Análise Descritiva dos Dados . . . . . . . . . . . . . . . . 34

Tabela 2 - Erros de Estimação . . . . . . . . . . . . . . . . . . . 53

Tabela 3 - Teste de Especificação - Horizonte Previsão de 1 mês* . . . . . . . . . 56

Tabela 4 - Teste de Especificação - Horizonte Previsão de 12 meses* . . . . . . . . 57

Tabela 5 - Teste de Especificação - Horizonte Previsão de 60 meses* . . . . . . . . 58 



\section{Introdução}

O entendimento da evolução da curva de juros desempenha papel de suma importância em diversas atividades, dentre as quais estão gestão de risco, precificação de ativos financeiros e seus derivativos, alocação de portfólios e estruturação de dívidas. Além disso, sua compreensão em conjunto com a atividade econômica é importante para condução da política monetária e fiscal do país.

Ao longo dos anos uma extensa literatura sobre sua estimação foi desenvolvida, em que três principais abordagens são utilizadas: modelos de não-arbitragem, modelos de equilíbrio e modelos estatísticos para a curva de juros. Dentre os modelos estatísticos uma classe importante é derivada do trabalho de Nelson-Siegel (1987), que será o elemento principal analisado neste trabalho. Modelos de não-arbitragem são importantes devido a sua consistência com restrições impostas pela teoria de apreçamento de ativos, mas em muitas vezes tem problemas em termos de ajuste aos dados observados e previsão fora da amostra. No que diz respeito aos modelos de equilíbrio, estes têm como foco a dinâmica da taxa instantânea, a partir da qual as taxas de juros de outras maturidades podem ser derivadas sob diversas hipóteses de prêmio de risco. De acordo com Duffee (2002) esta abordagem pode ser pouco adequada para a previsão fora da amostra.

No que diz respeito ao modelo de Nelson-Siegel (1987) e suas extensões, estes são reconhecidamente utilizados para a estimação e previsão da curva de juros, sendo amplamente empregados por bancos centrais, tais como o da Bélgica, Finlândia, França, Itália, Alemanha, Noruega, Espanha, Suécia, Suíça (e.g. Bank for International Settlements (2015)). Diebold e Li (2006) propõe um modelo dinâmico para a curva de juros de Nelson-Siegel (1987), em que cada parâmetro do ajuste cross-section do modelo de Nelson-Siegel é tratado como um fator latente, e por meio da modelagem e previsão destes fatores latentes é possível obter previsões para toda a estrutura a termo da taxa de juros. Os resultados obtidos em (DIEBOLD; LI, 2006) mostram que o ajuste e poder preditivo são superiores às demais metodologias para uma parte importante da curva de juros analisada.

Extensões do modelo dinâmico de Nelson-Siegel, as quais incorporam variáveis macroeconômicas (e.g. Diebold et al. (2006)), mudanças de regime discretas (e.g. Christensen (2015) e Hevia et al. (2015)) e/ou mudanças de regime contínuas (e.g. Zhu e Rahman (2015), Levant e Ma (2017) e Kobayashi (2017)) mostram uma melhora no ajuste e/ou previsão da estrutura a termo dos EUA e tem enriquecido a literarura. Além disso, o modelo dinâmico de Nelson-Siegel com variáveis macroeconômicas e mudanças de regime fornece grande flexibilidade para o entendimento das interações entre a curva de juros e a macroeconomia (e.g. Diebold e Rudebusch (2013)).

O comportamento conjunto da taxa de juros a termo e de variáveis macroeconô- 
micas é importante para associar eventos macroeconômicos com a dinâmica da curva de juros. Apesar disso, não há uma comparação direta das variáveis macroeconômicas com fatores latentes, os quais explicam os movimentos da curva de juros em diversos modelos de estrutura a termo. Também pela especificidade da curva de juros, que é determinada tanto pelo mercado de ativos quanto por fatores macroeconômicos, um modelo que incorpore esses dois mercados tende a gerar resultados melhores.

Dada a relevância do modelo de Nelson-Siegel e suas extensões na análise empírica das taxas de juros a termo, estimamos versões deste modelo para o mercado brasileiro incorporando diferentes métodos de mudanças de regime e variáveis macroeconômicas. Apesar de existirem artigos (e.g. Matsumura e Moreira (2006), Faria e Almeida (2014)) que incorporem tais variáveis utilizando dados brasileiros, não há trabalhos em que são empregadas mudanças de regime discretas e/ou contínuas ao modelo dinâmico de NelsonSiegel. Assim, temos como objetivo incorporá-las e verificar o ajuste e previsão da curva de juros. Não exclusivo para o mercado brasileiro, o método para a incorporação das variáveis latentes de mudança de regime com determinação do número de regimes feita por Monte Carlo via Cadeia de Markov (MCMC) com amostragem de Gibbs, conforme So et al. (1998), e a incorporação de mudança de regime tanto nas variáveis latentes quanto nas variáveis macroeconômicas é uma contribuição para a literatura de curva de juros.

Ademais, estimações e previsões fora da amostra de extensões do modelo dinâmico de Nelson-Siegel foram mais amplamente exploradas para dados dos EUA, que normalmente são mais bem comportadas devido a maior estabilidade econômica e política. O fato de serem propostas diferentes abordagens que incorporam mudanças de regime Markovianas à curva de DI são motivadas por evidências de mudanças de regime para dados da curva de juros dos EUA (e.g. Christensen (2015), Hevia et al. (2015), Zhu e Rahman (2015) e Levant e Ma (2017)) e para dados macroeconômicos brasileiros (e.g. Gonçalves et al. (2016), Arruda et al. (2011), Wichmann e Portugal (2013)). Além disso, o formato da curva de DI, conforme será visto, modifica-se bastante ao longo do tempo, o que dá indícios de que os fatores latentes devem apresentar maior variabilidade ao longo do tempo, o que torna mais interessante o emprego dos modelos aqui propostos.

Como a inferência estatística para este modelo é complicada por sua não-linearidade, já que o parâmetro de decaimento não é escolhido de maneira ad hoc, e por dois fatores não observados: os regimes latentes e os fatores latentes da curva de juros, utilizamos uma abordagem bayesiana de MCMC para sua estimação.

Os resultados mostram que para a inferência da curva de DI, os modelos com mudança de regime e incorporação de variáveis macroeconômicas apresentam melhor ajuste. Assim, este fato evidência que as abordagens propostas de mudanças de regime se mostram relevantes para a inferência com dados brasileiros. 
Para a previsão fora da amostra, os modelos com melhor poder preditivo dependem do horizonte de previsão e da maturidade analisada. Para o horizonte de previsão de 1 mês, a incorporação, principalmente, de fatores macroeconômicos e para maturidades mais elevadas a incorporação também de mudança de regime apresentam melhor poder preditivo. Para o horizonte de 12 meses, o modelo com mudança de regime, baseada no trabalho de So et al. (1998), nos fatores macroeconômicos se destaca. Já para o horizonte de 60 meses, o modelo com mudança de regime no fator de decaimento apresenta melhor poder preditivo, fator este que foi inicialmente escolhido de forma ad hoc para dados dos EUA no artigo Diebold e Li (2006). De modo geral, este trabalho mostra que diversos modelos tem melhor ajuste na estimação e uma melhor acurácia preditiva que o modelo dinâmico de Nelson-Siegel padrão para dados de DI.

A estrutura do trabalho foi feita da seguinte forma: na seção 2 apresentamos o referencial bibliográfico; na seção 3 apresentamos a metodologia utilizada para a modelagem, descrevendo o modelo de Nelson-Siegel (1987) com as modificações de Diebold e Li (2006) e incorporamos ao modelo a possibilidade de mudanças de regime e variáveis macroeconômicas. Na seção 4, é apresentada a base de dados utilizada. Já na seção 5, são apresentados os resultados das estimações econométricas. Por fim, na seção 6, discutimos os principais resultados e suas conclusões. 


\section{Referencial Bibliográfico}

O primeiro modelo de estrutura a termo da taxa de juros foi o de Vasiček, que pertence a classe dos modelos afins ou modelos de equilíbrio. A estrutura a termo afim captura o risco para títulos de longa maturidade em um mercado livre de arbitragem. Nestes modelos os preços dos títulos são afins na maturidade, ou seja, podem ser expressos por uma constante mais um termo linear. O modelo de Vasiček é baseado na evolução de uma taxa de juros de curto prazo não especificada e supõe que esta taxa segue a seguinte equação diferencial estocástica:

$$
d r_{t}=\alpha\left(\beta-r_{t}\right) d t+\sigma d W_{t}
$$

em que $\alpha, \beta$ e $\sigma$ são constantes não negativas, $r_{t}$ é o nível corrente da taxa de juros e $W_{t}$ é um movimento Browniano.

O modelo exibe reversão à média, isto é, se a taxa de juros está acima de sua média de longo prazo $(\mathrm{r}>\beta)$, o movimento se torna negativo e, assim, a taxa convirgirá na média para o nível $\beta$. O coeficiente $\alpha$ determina a velocidade com que a taxa de juros converge ao seu nível de longo prazo. Como neste caso a taxa de juros de curto prazo tem distribuição normal, para todo t haverá uma probabilidade positiva de que $\mathrm{r}$ seja negativa, o que é um problema do modelo, pois do ponto de vista econômico a taxa de juros assumir valores negativos não é razoável. Outra limitação deste modelo é que a volatilidade condicional de mudanças na taxa de juros é constante, ou seja, independente do nível de $r_{t}$.

Modelos bastante conhecidos nesta literatura, como de Cox, Ingersoll, Ross (CIR), Hull e White, Longstaff e Schwartz, pertencem a esta mesma classe e resolveram alguns dos problemas do modelo de Vasiček, conforme descrito a seguir. O modelo CIR pode ser definido como:

$$
d r_{t}=\alpha\left(\beta-r_{t}\right) d t+\sigma \sqrt{r_{t}} d W_{t}
$$

em que $\alpha, \beta$ e $\sigma$ são constantes não negativas, $r_{t}$ é o nível corrente da taxa de juros e $W_{t}$ é um movimento Browniano.

O fator $\sigma \sqrt{r_{t}}$ evita a possibilidade de taxas de juros negativas para todos os valores positivos de $\alpha$ e $\beta$. Quando é válida a condição $2 \alpha \beta \geq \alpha^{2}$ evita-se a possibilidade da taxa de juros assumir o valor zero. De forma mais geral, quando $r_{t}$ está próximo de zero, $\sigma \sqrt{r_{t}}$ se torna muito pequeno, o que diminui o efeito do choque aleatório.

Já no modelo de Hull e White de um fator, a taxa de juros de curto prazo segue o seguinte processo estocástico: 


$$
d r_{t}=\left(\frac{d \alpha_{t}}{d t}+\beta\left(\alpha_{t}-r_{t}\right)\right) d t+\sigma_{t} d W_{t}
$$

Neste processo a média reverte para um nível dependente no tempo, $\alpha_{t}$. Além disso, $\sigma$ também pode variar no tempo, permitindo que a volatilidade possa mudar de um período a outro.

Longstaff e Schwartz (1992) propõe um modelo da taxa de juros de curto prazo com dois fatores para que este represente o movimento da curva de juros de forma mais acurada, pois permite que os valores da taxa de juros reflitam tanto o seu nível corrente como sua volatilidade corrente. A dinâmica das variáveis de estado X e Y são governadas por:

$$
\begin{aligned}
& d X=(a-b X) d t+c \sqrt{X} d Z_{2} \\
& d Y=(d-e Y) d t+f \sqrt{Y} d Z_{3}
\end{aligned}
$$

$\mathrm{X}$ e Y são fatores econômicos, em que o X representa o componente dos retornos esperados. A vantagem de sua especificação é que os retornos esperados e a volatilidade não precisam ser perfeitamente correlacionados. E a, b, c, d, e, f $>0$, e $Z_{2}$ e $Z_{3}$ são processos de Wiener.

Assim como o modelo de Nelson-Siegel, diversas extensões dos modelos afins foram desenvolvidas. Um dos principais artigos dessa literatura é o de Ang e Piazzeni (2003), que estimam um modelo afim Gaussiano com a inclusão de variáveis macroeconômicas observáveis, o que permite o entendimento de sua dinâmica em conjunto com variáveis macroeconômicas e preços dos títulos. Encontram que os fatores macroeconômicos explicam uma porção significante de movimentos no curto e médio prazos da curva de juros e um porção menos significativa no longo prazo. Além disso, a inclusão das variáveis macroeconômicas também melhora a acurácia preditiva.

De acordo com Diebold et al. (2005), modelos afins puros de não arbitragem não fornecem uma compreensão das forças econômicas responsáveis pelos movimentos da curva de juros, mas com a incorporação de variáveis macroeconômicas é dada uma melhor compreensão dos determinantes fundamentais da curva de juros. Ainda segundo este artigo, de uma perspectiva macro, a taxa de juros a termo de curto prazo é um instrumento de política sob controle do Banco Central, que a ajusta para alcançar suas metas de estabilização econômica. Já pela perspectiva de finanças, a taxa de curto prazo é fundamental para a construção da curva para as demais maturidades pelo ajuste de risco.

Rudebusch e Wu (2008) também utilizam um modelo afim macro estrutural sem arbitragem com variáveis macroeconômicas e fatores latentes que determinam conjuntamente a curva de juros. Obtêm como resultado que choques externos tem um impacto 
significante em maturidades intermediárias e na curvatura, e surpresas inflacionárias tem um efeito significante no nível da curva de juros.

Para o mercado brasileiro, de acordo com Matsumura e Moreira (2006), a performance do modelo de Ang e Piazzeni (2003) não é satisfatória, o que gerou dúvidas quanto a efetividade da condição de não arbitragem e do prêmio afim para dados brasileiros. Apesar disso, a estimativa do modelo de Ang e Piazzeni para estes dados apresentou uma forte relação entre variáveis macro e financeiras com choques estruturais identificáveis, sendo capaz de avaliar o efeito de choques monetários na curva de juros.

Ainda com relação ao mercado brasileiro, Munclinger (2011) incorpora mudanças de regime no modelo afim de taxa de juros a termo. Neste trabalho a estimação do modelo é superior, dentro da amostra, em comparação ao modelo afim de regime único.

Com relação aos modelos de não-arbitragem, estes são representados principalmente pelo modelo de Heath-Jarrow-Morton (HJM), no qual desvios da não-arbitragem podem ser expressos como funções de suas volatilidades e das correlações entre si. Os modelos desenvolvidos de acordo com a estrutura geral de HJM são frequentemente nãomarkovianos e podem até ter dimensões infinitas. Para resolver estes dois problemas, vários trabalhos fizeram grandes contribuições, tais como o apresentado no artigo de Duffie e Kan (1996). Neste trabalho é desenvolvido um modelo multifatorial sem arbitragem, que pode ser visto como uma parametrização multifatorial de Markov do modelo HJM, mas também pode ser visto como uma versão multifatorial do modelo de fator único de CIR. Ao contrário da maioria dos modelos multifatorias de estrutura a termo, os fatores (variáveis que seguem um processo de Markov) são observáveis a partir da curva de juros e seus incrementos podem ter uma matriz de correlação especificada arbitrariamente.

Por fim, no que diz respeito a abordagem dos modelos de Nelson-Siegel, com o desenvolvimento de suas diferentes extensões, verificou-se uma melhora da acurácia preditiva da curva de juros e de seu entendimento em conjunto com outras variáveis de interesse. Este modelo propõe uma forma parcimoniosa de modelar a curva de juros capaz de reproduzir diversos fatos estilizados desta. A estrutura básica da curva de juros de Nelson-Siegel é dada por:

$$
y_{t}(\tau)=b_{1 t}+b_{2 t}\left(\frac{1-e^{-\lambda_{t} \tau}}{\lambda_{t} \tau}\right)-b_{3 t} e^{-\lambda_{t} \tau}+\epsilon_{t}(\tau)
$$

Em que t denota o tempo, $y_{t}(\tau)$ é a curva para um título com cupom zero que tem maturidade em $\tau$ meses, e $b_{1 t}, b_{2 t}, b_{3 t}$ e $\lambda_{t}$ são parâmetros e $\epsilon_{t}(\tau) \sim \mathrm{N}(0, \Omega)$.

Diebold e Li (2006) utiliza variações na estrutura dos componentes exponenciais de Nelson-Siegel (1987) para modelar toda a curva de juros, período a período, com três parâmetros variantes no tempo. Reinterpreta os três coeficientes da curva de juros de 
Nelson-Siegel como fatores latentes de nível, inclinação e curvatura e exploram a performance do modelo na previsão da curva de juros fora da amostra. Estimam modelos autoregressivos para os fatores e, com isso, projetam toda a curva de juros pela previsão dos fatores.

Com a reinterpretação dada por Diebold e Li (2006), esta curva tomou a seguinte forma:

$$
y_{t}(\tau)=\beta_{1 t}+\beta_{2 t}\left(\frac{1-e^{-\lambda_{t} \tau}}{\lambda_{t} \tau}\right)+\beta_{3 t}\left(\frac{1-e^{-\lambda_{t} \tau}}{\lambda_{t} \tau}-e^{-\lambda_{t} \tau}\right)+\epsilon_{t}(\tau)
$$

Obtida a partir do modelo de Nelson-Siegel (1987) quando $b_{1 t}=\beta_{1 t}, b_{2 t}=\beta_{2 t}+\beta_{3 t}$, e $b_{3 t}=\beta_{3 t}$. Este modelo proposto por Diebold e Li (2006) ficou conhecido como modelo dinâmico de Nelson-Siegel.

O parâmetro $\lambda$ é responsável pela taxa de decaimento exponencial e $\beta_{1 t}, \beta_{2 t}, \beta_{3 t}$ são os três fatores latentes dinâmicos. Estes $\beta$ s representam, respectivamente, os componentes de longo, curto e médio prazo. A evolução dos fatores latentes deste modelo é dada por um vetor autoregressivo de primeira ordem:

$$
\left[\begin{array}{l}
\beta_{1 t} \\
\beta_{2 t} \\
\beta_{3 t}
\end{array}\right]=\left[\begin{array}{l}
\mu_{1} \\
\mu_{2} \\
\mu_{3}
\end{array}\right]+\Phi\left[\begin{array}{l}
\beta_{1 t-1} \\
\beta_{2 t-1} \\
\beta_{3 t-1}
\end{array}\right]+\left[\begin{array}{l}
\eta_{1 t} \\
\eta_{2 t} \\
\eta_{3 t}
\end{array}\right]
$$

em que $\Phi$ denota a matriz $(3 \times 3)$ de parâmetros do vetor autoregressivo e $\eta \sim \mathrm{N}(0, \Omega)$.

Este modelo se tornou referência na previsão da estrutura a termo, pois obteve ajuste e poder preditivo superiores aos demais modelos da curva de juros, além de ser de fácil implementação, já que pode ser estimado por mínimos quadrados ordinários.

A estimação proposta por Diebold e Li (2006) é realizada por um procedimento em dois estágios. No primeiro estágio é estimada a equação (2) para cada dia observado, em que a estimação é feita por mínimos quadrados ordinários, o decaimento, $\lambda$, é assumido como fixo e conhecido, e os demais parâmetros são estimados para cada período de tempo t. No segundo estágio é realizada a estimação por mínimos quadrados ordinários da matriz de parâmetros $\Phi$ utilizando os $\beta_{1 t}, \beta_{2 t}$ e $\beta_{3 t}$ obtidos no primeiro estágio. Para realizar previsões é necessário estimar os fatores latentes t dias a frente e acrescentá-las na equação (2).

Os benefícios da estimativa de dois passos com $\lambda$ calibrado são a simplicidade, conveniência e estabilidade numérica, em que são necessárias somente regressões lineares. O custo desta estimação é a possível sub-otimalidade estatística, na medida em que o erro na estimação do parâmetro é ignorado no segundo passo, o que pode distorcer a inferência neste segundo estágio. 
Diversos outros procedimentos estão disponíveis para a estimação do modelo dinâmico de Nelson-Siegel, variando desde o procedimento em duas etapas, enunciado acima, à estimação por máxima verossimilhança exata usando representação em espaço-estado, dado pelas equações (2) e (3), em conjunto com o filtro de Kalman, até análises bayesianas utilizando métodos de Monte Carlo via Cadeia de Markov (MCMC).

A representação em espaço-estado do modelo facilita sua generalização para incorporar uma estrutura hierárquica em camadas, o que é útil para modelar curvas de juros conjuntas. Estas camadas surgem naturalmente em análises multinacionais, em que as curvas de juros de um país podem depender dos fatores do próprio país e estes fatores podem depender de fatores globais. Artigos de Diebold et al. (2008) e Laurini e Hotta (2009) fazem esta modelagem.

Nos artigos citados acima são estimadas um conjunto de curvas globais, que depende dos fatores globais latentes comuns de nível, inclinação e curvatura e de um fator idiossincrático global. O modelo pode ser representado por:

$$
Y_{t}(\tau)=\beta_{1 t}+\beta_{2 t}\left(\frac{1-e^{-\lambda_{t} \tau}}{\lambda_{t} \tau}\right)+\beta_{3 t}\left(\frac{1-e^{-\lambda_{t} \tau}}{\lambda_{t} \tau}-e^{-\lambda_{t} \tau}\right)+E_{t}(\tau)+\epsilon_{t}(\tau)
$$

em que, neste caso, $Y_{t}(\tau)$ são as curvas globais, $\beta_{1 t}, \beta_{2 t}$ e $\beta_{3 t}$ são os fatores comuns globais e $E_{t}(\tau)$ é o fator idiossincrático global. A dinâmica dos fatores comuns globais pode ser estimada por um processo autoregressivo, dado por:

$$
\left[\begin{array}{l}
\beta_{1 t} \\
\beta_{2 t} \\
\beta_{3 t}
\end{array}\right]=\left[\begin{array}{lll}
\Theta_{11} & \Theta_{12} & \Theta_{13} \\
\Theta_{21} & \Theta_{22} & \Theta_{23} \\
\Theta_{31} & \Theta_{32} & \Theta_{33}
\end{array}\right]\left[\begin{array}{c}
\beta_{1 t-1} \\
\beta_{2 t-1} \\
\beta_{3 t-1}
\end{array}\right]+\left[\begin{array}{c}
U_{t}^{\beta_{1 t}} \\
U_{t}^{\beta_{2 t}} \\
U_{t}^{\beta_{3 t}}
\end{array}\right]
$$

em que $U_{t}^{\beta_{1 t}}, U_{t}^{\beta_{2 t}}, U_{t}^{\beta_{3 t}}$ são choques de transição do estado global. Os fatores comuns globais do país, $l_{i t}$, $s_{i t}$ e $c_{i t}$, provêm dos fatores comuns globais $\beta_{1 t}, \beta_{2 t}$ e $\beta_{3 t}$, respectivamente. Este fato pode ser representado por:

$$
\begin{aligned}
& l_{i t}=\alpha_{i}^{l}+\gamma_{i}^{l} \beta_{1 t}+\eta_{i t}^{l} \\
& s_{i t}=\alpha_{i}^{s}+\gamma_{i}^{s} \beta_{2 t}+\eta_{i t}^{s} \\
& c_{i t}=\alpha_{i}^{c}+\gamma_{i}^{c} \beta_{3 t}+\eta_{i t}^{c}
\end{aligned}
$$

em que $\alpha_{i}^{l}$, $\alpha_{i}^{s}$ e $\alpha_{i}^{c}$ são constantes, $\gamma_{i}^{l}, \gamma_{i}^{s}$ e $\gamma_{i}^{c}$ são coeficientes dos fatores comuns globais, e $\eta_{i t}^{l}, \eta_{i t}^{s}, \eta_{i t}^{c}$ são erros estocásticos com média zero. 
No artigo de Diebold et al. (2008) são modeladas curvas de juros para Alemanha, Japão, Reino Unido e EUA, em que utilizam dados mensais de títulos do governo para extrair tais fatores. Concluem que os fatores de nível e inclinação globais existem e são importantes economicamente, representando uma fração significante das variações das curvas de juros de cada país. Ademais, chegam a conclusão que os fatores globais parecem estar ligados a fundamentos econômicos (inflação e atividade real). No entanto, apenas os componentes de nível e inclinação são utilizados para a especificação do modelo devido ao elevado número de parâmetros envolvidos na estimação.

Já o artigo de Laurini e Hotta (2009) estima por MCMC o modelo com todos os parâmetros, incluindo o referente a curvatura, além de incorporar condições de nãoarbitragem, utilizando dados de cupom cambial e eurodólares. Diferentemente do artigo de Diebold et al. (2008) são incluídos dados de países emergentes. Com este trabalho, encontram-se evidências de que não somente os fatores latentes evoluem no tempo, mas fatores de decaimento e volatilidade devem ser tratados como fatores latentes adicionais, que permitem procedimentos de ajuste e previsão mais precisos, especialmente para curvas de juros de países emergentes. Concluem que utilizando os métodos de estimação propostos é possível obter flexibilidade e não-arbitragem, permitindo generalizar as formulações livres de arbitragem para o ajuste simultâneo de múltiplas curvas de juros.

Para incorporar não-arbitragem ao modelo é necessário colocar um termo de ajuste à curva de juros. Christensen et al. (2011) mostra como o modelo dinâmico de NelsonSiegel pode ser ajustado para se enquadrar na classe Duffie-Kan de modelos de nãoarbitragem, os quais por definição não possibilitam ganho sem risco por diferença de preço correspondentes de um mesmo ativo com diferentes maturidades. O modelo com esta incorporação toma a seguinte forma:

$$
y(t, T)=X_{t}^{1}+\left(\frac{1-e^{-\lambda_{t} \tau}}{\lambda_{t} \tau}\right) X_{t}^{2}+\left(\frac{1-e^{-\lambda_{t} \tau}}{\lambda_{t} \tau}-e^{-\lambda_{t} \tau}\right) X_{t}^{3}-\frac{C(t, T)}{T-t}+\epsilon_{t}
$$

em que $X_{t}^{1}, X_{t}^{2}$ e $X_{t}^{3}$ são os fatores nível, inclinação e curvatura. A taxa de juros instantânea é a soma dos fatores de nível e inclinação, enquanto o fator de curvatura é como uma média estocástica variável no tempo para o fator de inclinação. Em particular, o termo de ajuste na curva para o modelo de não arbitragem do modelo dinâmico, $\frac{C(t, T)}{T-t}$, é a chave entre o modelo sem arbitragem e o modelo irrestrito. Este ajuste depende apenas da maturidade, não do tempo. O termo de ajuste é dado por:

$$
\begin{gathered}
\frac{C(t, T)}{T-t}=\bar{A}\left(\frac{(T-t)^{2}}{6}\right)+\bar{B}\left(\frac{1}{2 \lambda^{2}}-\frac{1}{\lambda^{3}} \frac{1-e^{-\lambda(T-t)}}{T-t}+\frac{1}{4 \lambda^{3}} \frac{1-e^{-2 \lambda(T-t)}}{T-t}\right) \\
+\bar{C}\left(\frac{1}{2 \lambda^{2}}+\frac{1}{\lambda^{2}} e^{-\lambda(T-t)}-\frac{1}{4 \lambda}(T-t) e^{-2 \lambda(T-t)}-\frac{3}{4 \lambda^{2}} e^{-2 \lambda(T-t)}-\frac{2}{\lambda^{3}} \frac{1-e^{-\lambda(T-t)}}{T-t}\right.
\end{gathered}
$$




$$
\begin{gathered}
\left.+\frac{5}{8 \lambda^{3}} \frac{1-e^{-2 \lambda(T-t)}}{T-t}\right) \\
+\bar{D}\left(\frac{1}{2 \lambda}(T-t)+\frac{1}{\lambda^{2}} e^{-\lambda(T-t)}-\frac{1}{\lambda^{3}} \frac{1-e^{-\lambda(T-t)}}{T-t}\right) \\
+\bar{E}\left(\frac{3}{\lambda^{2}} e^{-\lambda(T-t)}+\frac{1}{2 \lambda}(T-t)+\frac{1}{\lambda}(T-t) e^{-\lambda(T-t)}-\frac{3}{\lambda^{3}} \frac{1-e^{-\lambda(T-t)}}{T-t}\right) \\
+\bar{F}\left(\frac{1}{\lambda^{2}}+\frac{1}{\lambda^{2}} e^{-\lambda(T-t)}-\frac{1}{2 \lambda^{2}} e^{-2 \lambda(T-t)}-\frac{3}{\lambda^{3}} \frac{1-e^{-\lambda(T-t)}}{T-t}+\frac{3}{4 \lambda^{3}} \frac{1-e^{-2 \lambda(T-t)}}{T-t}\right)
\end{gathered}
$$

em que:

$$
\begin{gathered}
\bar{A}=\sigma_{11}^{2}+\sigma_{12}^{2}+\sigma_{13}^{2} \\
\bar{B}=\sigma_{21}^{2}+\sigma_{22}^{2}+\sigma_{23}^{2} \\
\bar{C}=\sigma_{31}^{2}+\sigma_{32}^{2}+\sigma_{33}^{2} \\
\bar{D}=\sigma_{11} \sigma_{21}+\sigma_{12} \sigma_{22}+\sigma_{13} \sigma_{23} \\
\bar{E}=\sigma_{11} \sigma_{31}+\sigma_{12} \sigma_{32}+\sigma_{13} \sigma_{33} \\
\bar{F}=\sigma_{21} \sigma_{31}+\sigma_{22} \sigma_{32}+\sigma_{23} \sigma_{33}
\end{gathered}
$$

E a matriz de volatilidade é dada por:

$$
\Sigma=\left(\begin{array}{lll}
\sigma_{11} & \sigma_{12} & \sigma_{13} \\
\sigma_{21} & \sigma_{22} & \sigma_{23} \\
\sigma_{31} & \sigma_{32} & \sigma_{33}
\end{array}\right)
$$

A incorporação deste termo de ajuste aumenta significativamente a complexidade do modelo. Ademais a isto, existem evidências empíricas que o modelo dinâmico de Nelson-Siegel quase não possui arbitragem sem a necessidade do termo de ajuste (e.g. Christensen et al. (2011)).

Para o modelo dinâmico de Nelson-Siegel, até agora exploramos variações quase que exclusivamente do ponto de vista da área de finanças, no entanto diversos aspectos da curva de juros estão ligados a características macroeconômicas, abrindo caminho para análises econômicas em macro-finanças. Pode-se proceder de uma perspectiva unilateral, em que se usa a curva de juros para explicar ou prever variáveis macroeconômicas ou utilizar as variáveis macroeconômicas para se prever a curva de juros, como Ang e Piazzeni (2003) fizeram para o modelo afim Gaussiano.

Embora os resultados baseados na perspectiva unidirecional sejam importantes, ainda são certamente deficientes, na medida em que interações dinâmicas entre fatores da 
curva de juros e fundamentos macroeconômicos traz ganhos adicionais, já que interações dinâmicas possivelmente envolvem causalidade bidirecional.

Um dos artigos que incorporam variáveis macroeconômicas ao modelo dinâmico de Nelson-Siegel é o de Diebold et al. (2006), o qual estima a curva de juros incorporando variáveis macroeconômicas observáveis - atividade real, inflação e instrumento de política monetária - utilizadas para capturar a dinâmica macroeconômica. Este artigo encontra forte evidência da causalidade bidirecional dos fatores da curva de juros em variáveis macroeconômicas e destas nos fatores da curva de juros.

Além disso, muitos trabalhos estudaram a relação dos fatores de nível, inclinação e curvatura com fundamentos macroeconômicos. Apesar de não ser possível relacionar fundamentos macroeconômicos a somente um destes fatores (nível, inclinação ou curvatura), a teoria econômica sugere que o nível da curva de juros nominal deveria estar relacionada ao nível de inflação esperada e, por isso, o trabalho de Diebold et al. (2006) leva em conta a inflação. Uma interpretação da teoria macroeconômica também sugere que a inclinação da curva de juros deveria estar relacionada com a atividade econômica real e, por isso, é levado em conta a capacidade de utilização manufatureira como fundamento macroeconômico em Diebold et al. (2006). Com relação a curvatura, argumenta-se que cabe a ela um papel limitado, assim Diebold et al. (2006) não inclui nenhum fundamento macroeconômico para direcionar a curvatura. Por fim, como a política macroeconômica pode afetar todos os fatores da curva de juros, Diebold et al. (2006) inclui uma variável para isso, a taxa de fundos federais.

Diversos artigos, discutidos no que segue, encontraram evidências empíricas de que o modelo dinâmico de Nelson-Siegel também teve uma melhora significativa com a incorporação de mudança de regime. Nestes casos, a ideia foi trocar a dinâmica linear básica dos fatores latentes, representados anteriormente pelos $\beta \mathrm{s}$, dada por:

$$
f_{t}=c+A f_{t-1}+\eta_{t}
$$

pela dinâmica de mudança de regime ao longo das linhas dada por:

$$
\begin{gathered}
f_{t}=c_{s_{t}}+A_{s_{t}} f_{t-1}+\eta_{t}, \\
\eta_{t} \sim N\left(0, \sigma_{s_{t}}^{2}\right)
\end{gathered}
$$

em que $s_{t}=1,2$, no caso de modelos de dois estados. A dinâmica linear padrão é um caso especial quando $c_{1}=c_{2}, A_{1}=A_{2}$ e $\sigma_{1}^{2}=\sigma_{2}^{2}$.

Além disso, existe o caso em que $s_{t}$ é um processo de Markov de primeira ordem, em que os regimes são determinados por um estado não observado ou variável de regime que segue um processo de Markov de estado discreto. Processos de Markov com estado 
discreto, chamados de cadeia de Markov, são muito populares para comportamentos de estado dependente. São aplicados para modelar e prever ciclos de negócios, estrutura a termo de taxas de juros, volatilidade em variáveis econômicas e financeiras, dinâmica de taxa de câmbio, dinâmica de taxa de inflação (e.g Diebold e Rudebusch (2013)). Assim, pode-se ir além, com a possibilidade de variação ao longo do tempo, por meio de uma matriz de transição de probabilidade, com $s_{t}$ guiado por: ${ }^{1}$

$$
P_{t}=\left(\begin{array}{cc}
p_{11, t} & 1-p_{11, t} \\
1-p_{22, t} & p_{22, t}
\end{array}\right)
$$

em que $p_{11, t}$ e $p_{22, t}$ são as probabilidades de mudança de regime em cada período.

Um dos trabalhos que incorpora mudança de regime é o de Hevia et al. (2015), o qual utiliza um modelo com variável latente de mudança de Markov que permite saltos no processo estocástico seguido pelas taxas de juros. Derivam também restrições de nãoarbitragem para tempo discreto para este modelo. Hevia et al. (2015) avalia também se quebras na curva de juros advindas de variações no parâmetro de decaimento, $\lambda$, podem ser capturadas utilizando um modelo com mudança de regime para evitar potencial sobreajuste dos dados, além de avaliar em qual extensão é possível incorporar restrições de nãoarbitragem na estrutura de mudança de Markov e ao mesmo tempo possibilitar variações na forma da curva de juros ao longo do tempo.

Concluem que o modelo de regime único, que trata $\lambda$ como um fator contínuo variante no tempo, tem uma performance muito boa em ajuste e previsão somente em um horizonte de curtíssimo prazo e, dentre os modelos com mudança de Markov, a imposição de não-arbitragem é importante somente no curto e médio prazo. Por outro lado, modelos com mudança no parâmetro de decaimento tem uma boa performance na previsão nos horizontes de médio e longo prazo. Com isso, a performance do modelo depende do horizonte de previsão, em que vários deles se mostram melhores que o modelo dinâmico de Nelson-Siegel de regime único.

O trabalho de Zhu e Rahman (2015), além de incluir mudança de regime na estrutura a termo do modelo dinâmico de Nelson-Siegel, inclui também os fatores macroeconômicos, já incorporados em textos como o de Diebold et al. (2006). Para isso utilizam um procedimento de MCMC. Verificam que fatores macroeconômicos ajudam a explicar movimentos da curva de juros e melhoram a performance de previsão desta. Ademais, como a estrutura a termo da taxa de juros contém informações importantes sobre a atividade econômica, a curva de juros com fatores macroeconômicos ajuda também a explicar problemas macroeconômicos e produzem previsões de crescimento de PIB superiores. O método MCMC permite estimar o modelo com eficiência e extrair os fatores latentes da

\footnotetext{
1 No caso de dois regimes.
} 
curva de juros e os regimes não observados simultaneamente. Concluem que mudanças de regime são importantes para entender a interação entre a curva de juros e a atividade econômica.

Christensen (2015) inclui mudança de regime em um modelo mais adequado para a taxa de juros a termo dos EUA, pelo fato de que sua política monetária atingiu seu limite inferior (zero efetivo) desde dezembro de 2008. Diferente dos artigos de mudança de regime de Zhu e Rahman (2015) e Hevia et al. (2015), Christensen estima um modelo com um estado normal e outro estado com limite no zero. Para gerar variação mesmo nas taxas de curto prazo, é dada uma chance positiva de saída do limite zero a qualquer tempo, que é modelado a partir de um processo variante no tempo para um processo de Poisson no ponto, cujo primeiro salto indica a saída do limite zero. Para o estado normal, a curva de juros é modelada utilizando a versão de não-arbitragem do modelo de Nelson-Siegel desenvolvida por Christensen et al. (2011). Assume-se que a economia volta ao estado normal quando sai do estado com limite no zero, representando uma mudança na estrutura dinâmica na curva de juros e não somente uma mudança discreta.

Artigos mais recentes, (LEVANT; MA, 2017) e (KOBAYASHI, 2017), também incluem mudanças de regime ao modelo dinâmico de Nelson-Siegel, no entanto se concentram somente na análise do ajuste dentro da amostra da curva de juros. O primeiro utiliza dados dos EUA e verifica que a inclusão de mudanças de regime no fator de decaimento melhora a previsão dentro da amostra quando comparado tanto ao modelo dinâmico de Nelson-Siegel padrão quanto deste com a inclusão de mudança de regime na volatilidade. Já o artigo de Kobayashi (2017) utiliza dados japoneses de títulos corporativos e incorpora uma matriz de transição de probabilidade guiada por indicadores macroeconômicos, o que melhora o ajuste da curva de juros.

É importante ressaltar que o Brasil possui características distintas do mercado financeiro dos EUA, com uma menor liquidez de títulos e maior número de intervenções, o que pode ocasionar mudanças de regime mais frequentes e maior volatilidade dos preços devido a incertezas macroeconômicas. Ademais, para o mercado brasileiro, diversos trabalhos (e.g. Gonçalves et al. (2016), Arruda et al. (2011), Wichmann e Portugal (2013)) encontram que mudanças de regime tem um ajuste superior para estimação de parâmetros para política monetária, Regra de Taylor e principais parâmetros da economia brasileira utilizados em modelos DSGE.

No entanto, mesmo no caso brasileiro, artigos encontram evidências empíricas de que o modelo dinâmico de Nelson-Siegel e suas extensões possuem maior poder de previsão comparado a modelos de estrutura a termo afim e ao passeio aleatório (e.g. Vicente e Tabak (2008), Laurini e Hotta (2010), Matsumura e Moreira (2006), Caldeira et al. (2016)). 
Com a introdução de fatores macroeconômicos ao modelo dinâmico de NelsonSiegel, Faria e Almeida (2014) verificaram uma melhora significativa para horizontes maiores do que 3 meses, sem perdas significantes para horizontes de 1 mês, quando comparado ao modelo dinâmico sem fatores macroeconômicos.

Com base nos artigos citados acima, vemos a importância que o trabalho de NelsonSiegel com a extensão dada por Diebold e Li (2006) teve ao longo dos últimos anos para a estimação e previsão da estrutura a termo da taxa de juros. Além da importância que projeções com mudança de regime tem tomado na literatura nos anos mais recentes. Logo, este trabalho se propõe a estimar o modelo dinâmico levando em conta estas mudanças de regime e verificar se com a incorporação de mudanças de regime em variáveis macroeconômicas, os modelos apresentam uma melhora na acurácia preditiva e ajuste na estimação.

No caso brasileiro, modelos de taxa de juros a termo do tipo Nelson-Siegel com macro-fatores e a incorporação de mudanças de regimes ainda não foram estimados. Além disso, modelos com mudança de regime tanto na variável latente quanto nas variáveis macroeconômicas e a utilização do método MCMC com amostragem de Gibbs, tal como no traballho de So et al. (1998), também contribuem para a literatura. 


\section{Metodologia}

Esta seção descreve diferentes extensões do modelo dinâmico de Nelson-Siegel que empregamos para parametrizar a curva de juros. A estrutura básica da curva de juros utilizada para estimação é dada pelas equações (2) e (3) especificadas no capítulo anterior

Foram estimados modelos que incorporam ao modelo dinâmico de Nelson-Siegel mudança de regime em que as probabilidades seguem um processo de Markov e a matriz de transição segue o método descrito em (12). São propostos modelos, em que estas mudanças são incorporadas na média, na persistência e em conjunto com mudanças de regime nas variáveis macroeconômicas.

Além disso, foi utilizada a metodologia presente no texto de So et al. (1998), descrita a seguir. Dessa forma, generalizou-se o modelo dinâmico de Nelson-Siegel para incorporar variáveis macroeconômicas e mudanças de regime, permitindo ocasionais mudanças discretas (saltos) e também mudanças contínuas em sua estrutura.

A extensão macroeconômica do modelo dinâmico de Nelson-Siegel possui três fatores macro observáveis: capacidade de utilização manufatureira $(\mathrm{CU})$, a taxa de fundos federais (FFR) e a inflação (INFL), que segundo Diebold et al. (2006), representam, respectivamente, um fator real para capturar a atividade econômica real, um fator de política monetária para capturar o efeito da política e, por fim, um fator para descrever o nível de preços. Assim, representamos as variáveis de estado em um vetor $(7 \times 1), X_{t}^{\prime}=\left(\beta_{1 t}\right.$ $\left.\beta_{2 t} \beta_{3 t} \lambda_{t} C U_{t} F F R_{t} I N F L_{t}\right)$ e $\mathbf{y}_{t}^{\prime}=\left(\begin{array}{lll}y_{t\left(\tau_{1}\right)} & \ldots & \left.y_{t\left(\tau_{n}\right)}\right)\end{array}\right)$, de modo que o modelo pode ser sucintamente escrito como:

$$
\mathbf{y}_{t}=\Lambda X_{t}+\epsilon_{t}
$$

onde $\Lambda$ é uma matriz de coeficientes $(\mathrm{N} \times 7)^{2}$.

Para possibilitar as mudanças de estado do modelo, de acordo com (SO et al., 1998), suponha que $s_{t}$ é uma variável aleatória discreta com domínio $1,2, \ldots, \mathrm{K}$ e assuma um processo de Markov de primeira ordem estado-K. Assim, podemos escrever a matriz de transição como:

$$
\left(\begin{array}{cccc}
p_{11} & p_{12} & \ldots & p_{1 K} \\
p_{21} & p_{22} & \ldots & p_{2 K} \\
\cdot & \cdot & & \cdot \\
\cdot & \cdot & \ldots & \cdot \\
p_{K 1} & p_{K 2} & \ldots & p_{K K}
\end{array}\right)
$$

2 As três colunas a direita contêm somente zeros, então a curva de juros irá manter somente os fatores latentes, o que é consistente com a visão de que somente os três fatores latentes são necessários para obtenção de toda a informação da curva de juros (e.g. Diebold et al. (2006)). 
em que $p_{i j}=\operatorname{Pr}\left(s_{t}=j \mid s_{t-1}=i\right) \operatorname{com} \sum_{j=1}^{K} p_{i j}=1$ para $\mathrm{i}=1, \ldots, \mathrm{K}$ e a variável de estado $s_{t}$ define um regime particular para os valores dos parâmetros. Por exemplo, $s_{t}=1$ corresponde ao primeiro regime e $s_{t}=K$ corresponde ao último regime. A equação de estado com mudança de regime é dada por:

$$
X_{t+1}=\alpha_{s_{t+1}}+\phi X_{t}+\eta_{t}
$$

em que:

$$
\alpha_{s_{t}}=\gamma_{1}+\sum_{j=2}^{K} \gamma_{j} I_{j t}
$$

e $I_{j t}$ é uma variável que é igual a 1 quando $s_{t}$ é maior ou igual a j.

Equações (13), (15) e (16) juntas definem o modelo com mudança de Markov estado-K. A dinâmica de mudança é governada por um processo de Markov de primeira ordem. Desta forma, este modelo captura simultaneamente a mudança no comportamento devido a forças econômicas como também mudanças abruptas discretas devido a eventos atípicos, já que permite diferentes $\alpha$ em diferentes regimes, que podem capturar ocasionais mudanças discretas (saltos).

Adicionalmente são feitas as seguintes hipóteses: (i) o coeficiente $\phi$ deve estar dentro do círculo unitário; (ii) cada regime deve corresponder a pelo menos um ponto no tempo; e (iii) todo $\gamma_{j}(\mathrm{j}=2, \ldots, \mathrm{K})$ é negativo. A hipótese (i) garante estacionariedade dado o estado $s_{t}$, a hipótese (ii) garante que os $\gamma_{j}$ 's não serão não-identificados. Por fim, a hipótese (iii) implica que quanto maior o valor de $s_{t}$, menor o nível. Dessa forma, o primeiro regime define o estado com maior nível e o último regime define o estado com menor nível.

Amostras da distribuição conjunta posterior dos parâmetros desconhecidos, assim como das variáveis latentes, são gerados por meio da amostragem de Gibbs (algoritmo para gerar uma sequência de amostras da distribuição conjunta de probabilidades de duas ou mais variáveis aleatórias).

Desta forma, adotaremos o MCMC e o aumento artificial do volume de dados disponíveis (data augmentation) que são combinados para a estimação dos parâmetros, e os fatores latentes são baseados no algoritmo amostral de Gibbs.

Neste contexto, o uso de métodos bayesianos de estimação usando algoritmos de MCMC permite a estimação utilizando toda a informação disponível na estrutura a termo de taxas de juros e evitando a imposição de restrições ad hoc. Esta metodologia permite tratar os problemas existentes dos mecanismos usuais de estimação, como os problemas de não-linearidade, identificação, e dimensionalidade envolvidos. Na estimação por MCMC, 
modelos lineares e não-lineares são tratados da mesma forma, e uma vantagem da metodologia bayesiana é que ela permite tratar os fatores latentes como parâmetros adicionais a serem estimados.

Em inferência bayesiana o objetivo é encontrar a chamada distribuição posterior dos parâmetros de interesse condicionados a amostra observada, denotada por $\mathrm{p}(\Theta \mid \mathrm{y})$. Esta distribuição a posteriori é o resultado da atualização da distribuição a priori assumida para os parâmetros com a informação existente na amostra, representada pela função de verossimilhança. Para encontrar a distribuição dos parâmetros condicionados a amostra, usa-se a seguinte equação derivada do lema de Bayes:

$$
p(\Theta \mid y)=p(\Theta, y) / p(y)=p(y \mid \Theta) p(\Theta) / p(y)
$$

em que $\mathrm{p}(\mathrm{y} \mid \Theta)$ é a verossimilhança do modelo, $\mathrm{p}(\Theta)$ denota a distribuição a priori assumida para o parâmetro e $\mathrm{p}(\mathrm{y})$ é a distribuição marginal da amostra, que precisa ser conhecida até uma constante de integração. Isto pode ser representado por:

$$
p(\Theta \mid y)=p(\Theta, y) / p(\Theta)=p(y \mid \Theta) p(\Theta) / c
$$

Desta forma, a distribuição a posteriori é proporcional ao produto da verossimilhança pela distribuição a priori:

$$
p(\Theta \mid y) \propto p(y \mid \Theta) p(\Theta)
$$

Logo, o objetivo principal da estimação bayesiana é obter a distribuição a posteriori, que contém a informação a priori atualizada pela informação existente na amostra dada pela função de verossimilhança.

Uma metodologia de Monte Carlo fundamental em métodos de estimação bayesiana é o uso dos chamados algoritmos de Monte Carlo via Cadeia de Markov (MCMC). Os métodos de MCMC simulam uma cadeia de Markov cuja distribuição estacionária converge para a distribuição $\mathrm{p}(\Theta \mid \mathrm{y})$. Um resultado fundamental é que a estimação de $\mathrm{p}(\Theta \mid \mathrm{y})$ pode ser fatorada, utilizando um método de amostragem das distribuições condicionais dos parâmetros. Estas condicionais são de dimensão inferior e podem ser mais facilmente simuladas.

Um benefício deste método é que não envolve nenhuma metodologia de maximização numérica, e, desta forma, evita-se problemas em realizar a maximização de funções não lineares. A validade empírica desta metodologia é verificada por meio de métodos para apurar a convergência das cadeias de Markov para a sua distribuição estacionária. Outra 
vantagem de métodos de inferência bayesiana é que o uso de informação a priori ajuda a resolver alguns problemas, como, por exemplo, a estimação de modelos não-identificados.

Com isso, vemos as vantagens da estimação por MCMC nos modelos aqui considerados. 


\section{Base de Dados}

Para este trabalho utilizaremos dados de fechamento mensais (último dia de cada mês) das estruturas a termo das taxas de juros dos contratos DI com diferentes maturidades (um, três, seis, nove, doze, quinze, dezoito, vinte, vinte e quatro, vinte e sete, trinta, trinta e três, trinta e seis, trinta e nove, quarenta e oito, sessenta e setenta e dois meses), com dados disponíveis na BM\&FBovespa ${ }^{3}$ de fevereiro de 2003 a agosto de 2017 . Foram incorporadas variáveis macroeconômicas com dados mensais, seguindo o padrão de Diebold et al. (2006), para capacidade de utilização manufatureira (utilização da capacidade instalada - indústria), a taxa de fundos federais (Selic) e taxa de inflação (IPCA). Dados estes disponíveis na Confereção Nacional da Indústria, Banco Central do Brasil e IBGE, respectivamente, de fevereiro de 2003 a agosto de 2017.

A utilização de dados de títulos com cupom zero elimina o efeito-cupom, que se refere a situação em que dois títulos idênticos tem diferentes taxas de cupom e diferentes taxas na maturidade.

Na figura 1 são apresentados os dados observados de DI nas diferentes maturidades. As variações temporais, principalmente de inclinação e curvatura, são visíveis neste gráfico. Na tabela 1, estão as análises das estatísticas descritivas da curva de juros. Tanto a inclinação quanto a curvatura possuem baixo desvio padrão quando comparados às taxas de juros a termo individuais, também se mostram menos persistentes que estas.

O padrão apresentado pela figura 1 torna a sua análise interessante para os modelos propostos. Nota-se que existe uma grande modificação no formato das curvas de juros no tempo, indicando que os fatores latentes devem ter grande variabilidade. Outro ponto é o fato de ser evidente que o padrão de decaimento da curva se modifica bastante no tempo, justificando o uso de parâmetros variantes no tempo em oposição a fixação realizada na estimação usual do modelo de Diebold e Li (2006).

Na figura 2 são apresentadas as medianas para cada maturidade. Nota-se que mesmo as taxas de juros de longa maturidade apresentam movimentos oscilatórios, o que é mais comum em países em desenvolvimento, pois em países desenvolvidos as maturidades de longo prazo tendem a ser mais estáveis devido a sua maior estabilidade econômica. Ademais, pela figura 3, nota-se que os dados macroeconômonicos também apresentam variabilidade ao longo do tempo, o que torna o emprego dos modelos com mudanças de regime mais interessante.

3 <http://www.bmfbovespa.com.br/pt_br/servicos/market-data/consultas/mercado-de-derivativos/ precos-referenciais/taxas-referenciais-bm-fbovespa/> 
Figura 1 - Gráfico dos Dados Observados de DI para as 17 diferentes Maturidades

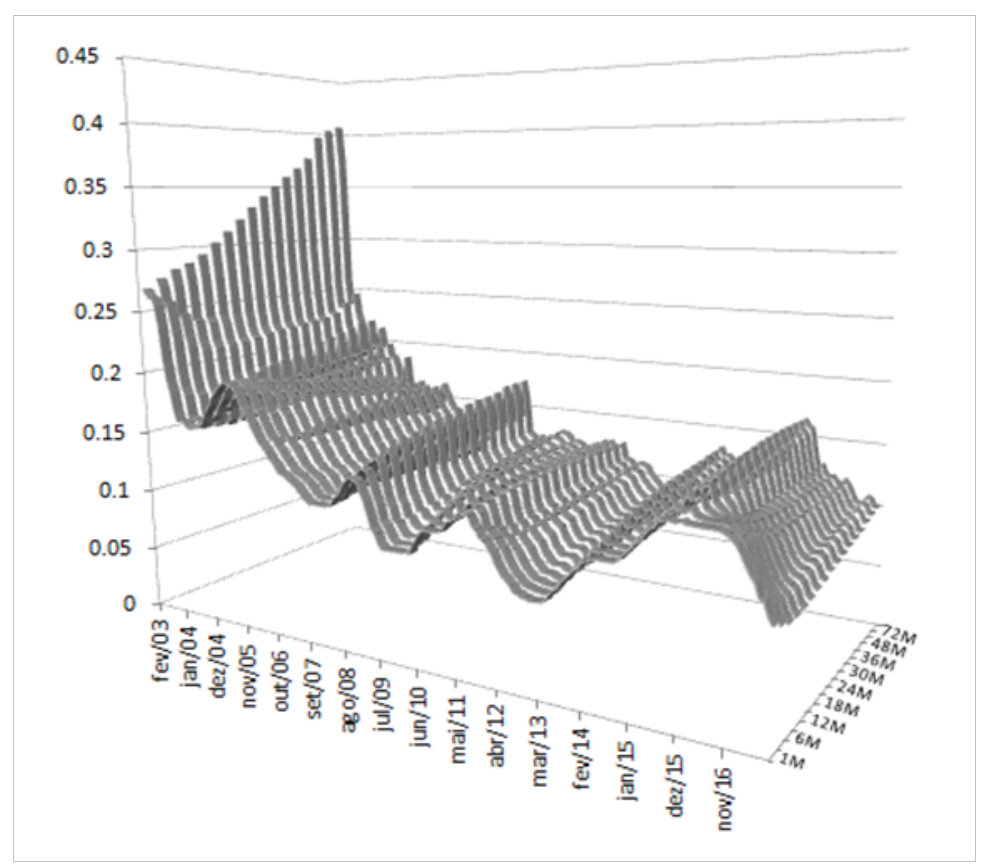

Tabela 1 - Análise Descritiva dos Dados

\begin{tabular}{lccccccc}
\hline Maturidade (meses) & Média & dvp & Máximo & Mínimo & $\rho(1)$ & $\rho(12)$ & $\rho(24)$ \\
\hline $1 \mathrm{M}$ & 0.1293 & 0.0404 & 0.2682 & 0.0697 & 0.9561 & 0.4454 & 0.3688 \\
$3 \mathrm{M}$ & 0.1290 & 0.0397 & 0.2758 & 0.0704 & 0.9487 & 0.4421 & 0.3446 \\
$6 \mathrm{M}$ & 0.1290 & 0.0388 & 0.2825 & 0.0707 & 0.9389 & 0.4386 & 0.3193 \\
$9 \mathrm{M}$ & 0.1292 & 0.0380 & 0.2866 & 0.0708 & 0.9313 & 0.4293 & 0.2993 \\
$12 \mathrm{M}$ & 0.1299 & 0.0375 & 0.2933 & 0.0715 & 0.9232 & 0.4179 & 0.2854 \\
$15 \mathrm{M}$ & 0.1306 & 0.0371 & 0.3026 & 0.0722 & 0.9130 & 0.4061 & 0.2775 \\
$18 \mathrm{M}$ & 0.1314 & 0.0368 & 0.3118 & 0.0740 & 0.9024 & 0.3965 & 0.2722 \\
$21 \mathrm{M}$ & 0.1320 & 0.0366 & 0.3217 & 0.0759 & 0.8915 & 0.3893 & 0.2683 \\
$24 \mathrm{M}$ & 0.1327 & 0.0366 & 0.3324 & 0.0776 & 0.8799 & 0.3816 & 0.2646 \\
$27 \mathrm{M}$ & 0.1333 & 0.0367 & 0.3418 & 0.0791 & 0.8700 & 0.3764 & 0.2605 \\
$30 \mathrm{M}$ & 0.1337 & 0.0369 & 0.3509 & 0.0800 & 0.8611 & 0.3735 & 0.2563 \\
$33 \mathrm{M}$ & 0.1342 & 0.0371 & 0.3593 & 0.0810 & 0.8533 & 0.3724 & 0.2515 \\
$36 \mathrm{M}$ & 0.1346 & 0.0374 & 0.3673 & 0.0820 & 0.8458 & 0.3700 & 0.2459 \\
$39 \mathrm{M}$ & 0.1350 & 0.0378 & 0.3768 & 0.0830 & 0.8379 & 0.3665 & 0.2391 \\
$48 \mathrm{M}$ & 0.1360 & 0.0392 & 0.3965 & 0.0845 & 0.8286 & 0.3548 & 0.2230 \\
$60 \mathrm{M}$ & 0.1370 & 0.0409 & 0.4034 & 0.0868 & 0.8352 & 0.3467 & 0.2075 \\
$72 \mathrm{M}$ & 0.1378 & 0.0420 & 0.4072 & 0.0881 & 0.8371 & 0.3406 & 0.2072 \\
Inclinação & -0.0085 & 0.0235 & 0.0430 & -0.1391 & 0.7756 & 0.0140 & -0.0696 \\
Curvatura & -0.0013 & 0.0152 & 0.0314 & -0.0823 & 0.8054 & 0.0211 & 0.0698 \\
\hline
\end{tabular}

A inclinação é definida como a diferença entre a taxa de juros a termo com maturidade de 1 mês e de 72 meses, e a curvatura é definida como duas vezes a taxa de juros a termo com maturidade de 24 meses menos a soma das taxas de maturidade de 3 meses e 72 meses. São apresentadas as autocorrelações para 1, 12 e 24 meses. 
Figura 2 - Gráfico da Mediana para cada uma das Maturidades

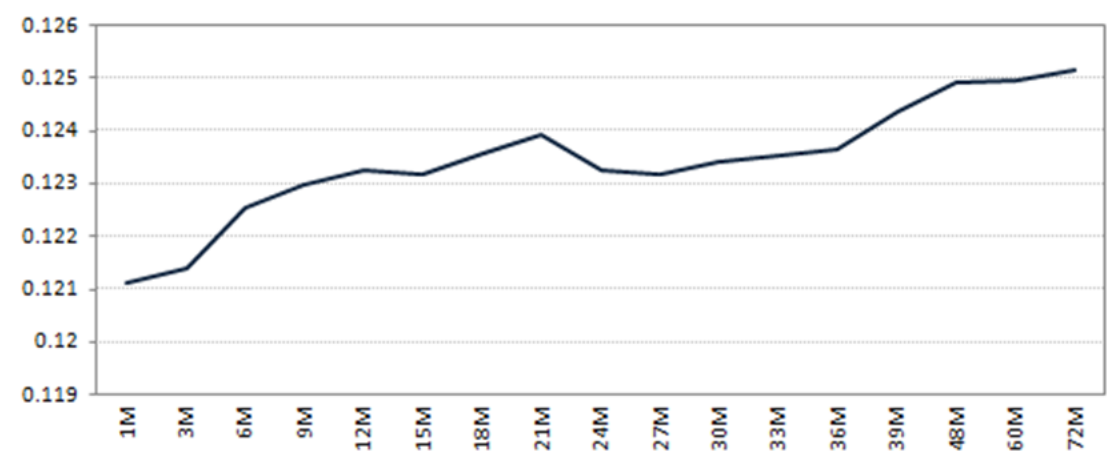

Figura 3 - Gráfico Dados Macroeconômicos

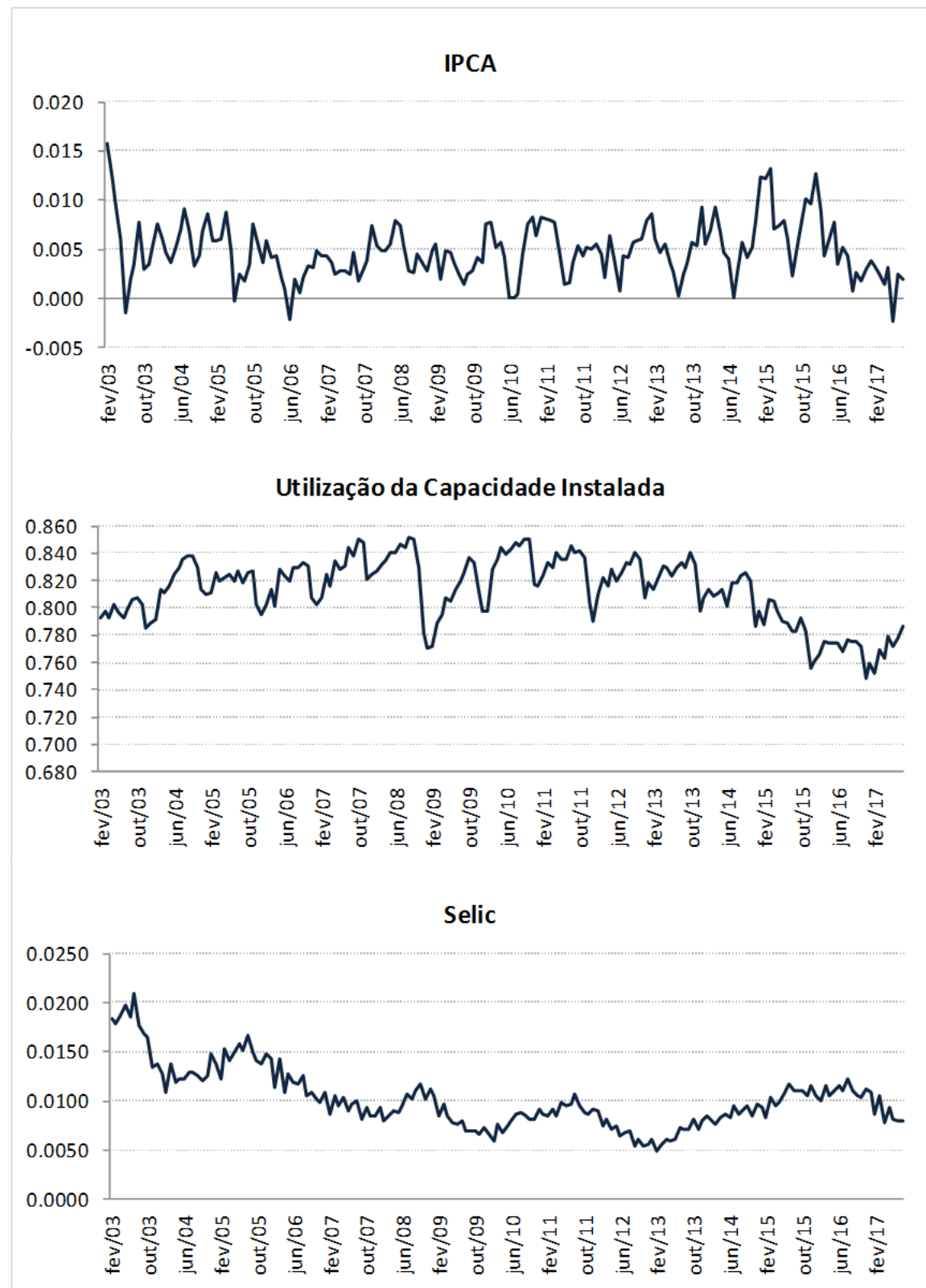




\section{Resultados}

Foram estimadas diversas versões do modelo dinâmico de Nelson-Siegel (MDNS) para a estrutura a termo da taxa de juros brasileira com a incorporação de variáveis macroeconômicas e/ou mudanças de regime no processo estocástico. Foram estimadas 13 especificações diferentes que são detalhadas abaixo:

1. Modelo Dinâmico de Nelson-Siegel (MDNS);

2. Modelo Dinâmico de Nelson-Siegel com mudança de regime na média (MDNS$\mathrm{M})$;

3. Modelo Dinâmico de Nelson-Siegel com mudança de regime na persistência (MDNS-P);

4. Modelo Dinâmico de Nelson-Siegel com mudança de regime no fator de decaimento (MDNS- $\lambda$ );

5. Modelo Dinâmico de Nelson-Siegel com a inclusão de variáveis macroeconômicas de forma exógena (MDNS-Macro);

6. Modelo Dinâmico de Nelson-Siegel com mudança de regime na média e com a inclusão de variáveis macroeconômicas de forma exógena (MDNS-MMacro);

7. Modelo Dinâmico de Nelson-Siegel com mudança de regime na persistência e com a inclusão de variáveis macroeconômicas de forma exógena (MDNS-PMacro);

8. Modelo Dinâmico de Nelson-Siegel com a inclusão de variáveis macroeconômicas de forma endógena e mudança de regime na média e nas variáveis macroeconômicas (MDNS-MMacroEnd);

9. Modelo Dinâmico de Nelson-Siegel com a inclusão de variáveis macroeconômicas de forma endógena e mudança de regime na persistência e nas variáveis macroeconômicas (MDNS-PMacroEnd);

10. Modelo Dinâmico de Nelson-Siegel com mudança de regime com a especificação de So et al. (1998) na média (MDNS-S);

11. Modelo Dinâmico de Nelson-Siegel com mudança de regime com a especificação de So et al. (1998) no fator de decaimento (MDNS-S $\lambda$ );

12. Modelo Dinâmico de Nelson-Siegel com mudança de regime com a especificação de So et al. (1998) na média e com a inclusão de variáveis macroeconômicas (MDNSSmediaMacro);

13. Modelo Dinâmico de Nelson-Siegel com mudança de regime com a especificação de So et al. (1998) nas variáveis macroeconômicas do fator de inclinação (MDNS-Smacro).

Estas especificações permitem analisar como as diferentes características dos mo- 
delos afetam o ajuste e os resultados obtidos. O método utilizado para a estimação em todas as abordagens foi o MCMC, no qual foram realizadas no mínimo 100.000 iterações para se chegar à distribuição condicional, com burn-in (número de amostras descartadas) de 10.000, e 90.000 iterações para a construção das distribuições posteriores. A família considerada na distribuição das priores dos fatores latentes, $\beta \mathrm{s}$ e $\lambda$, foi a gaussiana e log-gaussiana e das variâncias foi a gamma. Para as probabilidades, quando foi incluída a mudança de regime contínua, a família considerada foi a beta. Já para as probabilidades da mudança de regime discreta foi considerada a distribuição uniforme. No que diz respeito aos hiperparâmetros utilizados, podem ser obtidos com a autora.

Primeiramente, foi estimado o modelo dinâmico de Nelson-Siegel (MDNS) apenas com os fatores latentes, para as 17 maturidades descritas anteriormente. As variáveis latentes obtidas a cada período são apresentadas na figura 4 .

\section{Figura 4 - Gráfico Variáveis Latentes MDNS}

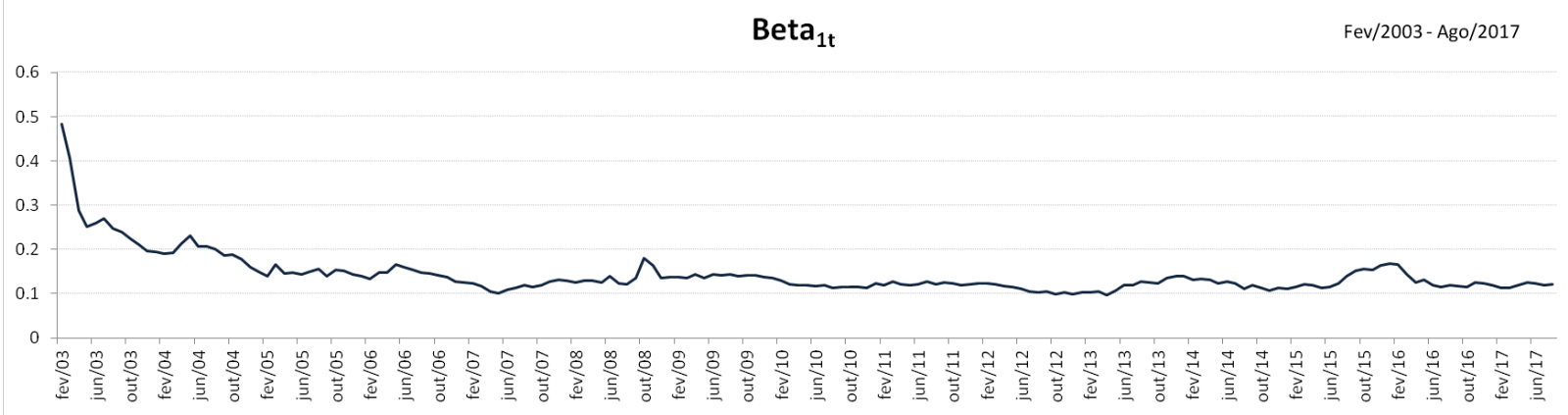

Beta $_{2 t}$

$\mathrm{Fev} / 2003$ - Ago/2017
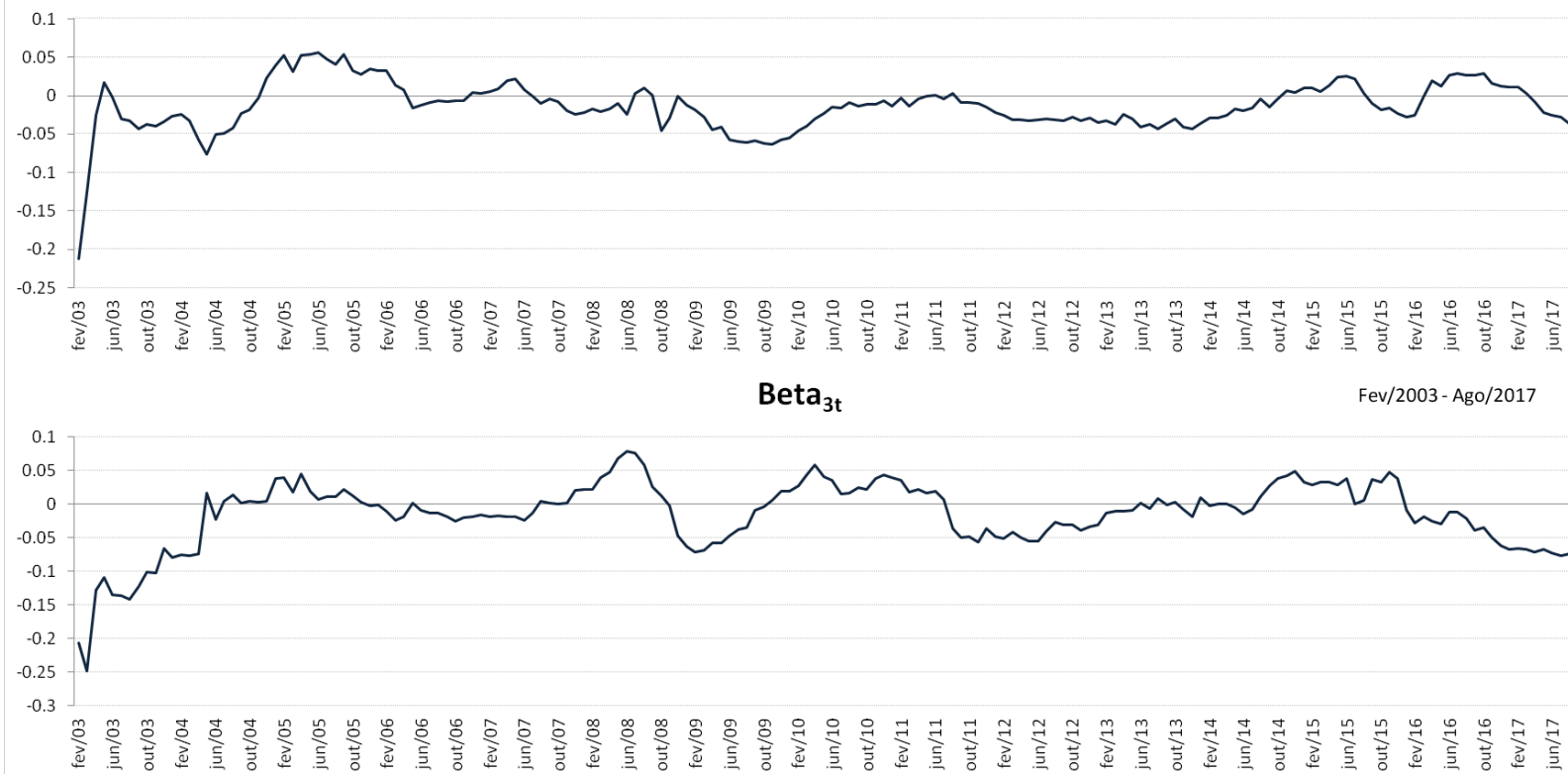

No segundo modelo estimado foi incorporada mudança de regime na média (MDNSM), em que é feita a seguinte modificação no MDNS: 


$$
\beta_{2 t}=\mu_{s t}+\phi_{21}\left(\beta_{2 t-1}-\mu_{s t}\right)
$$

em que s pode assumir o valor de 1 ou 2, conforme o regime. As variáveis latentes estimadas são apresentadas no gráfico 5 .

\section{Figura 5 - Gráfico Variáveis Latentes MDNS-M}

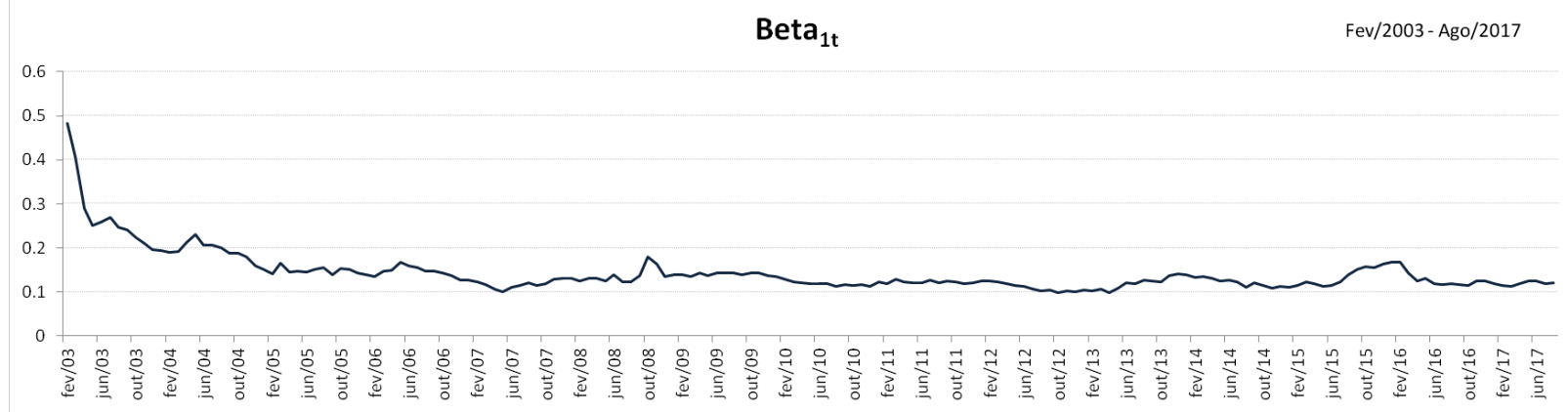

Beta $_{2 \mathrm{t}}$

$\mathrm{Fev} / 2003$ - Ago/2017

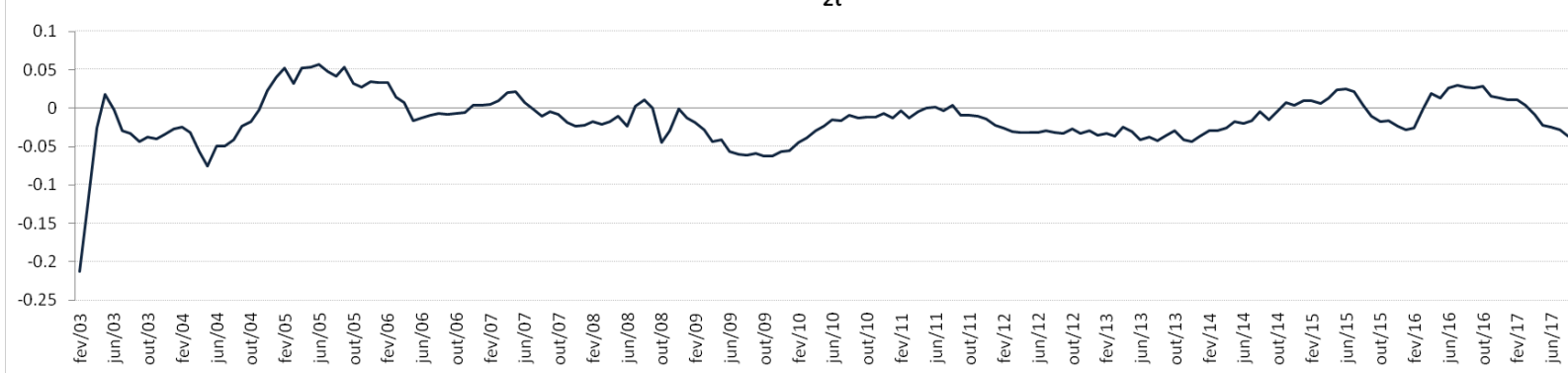

Beta $_{3 t}$

$\mathrm{Fev} / 2003$ - Ago/2017

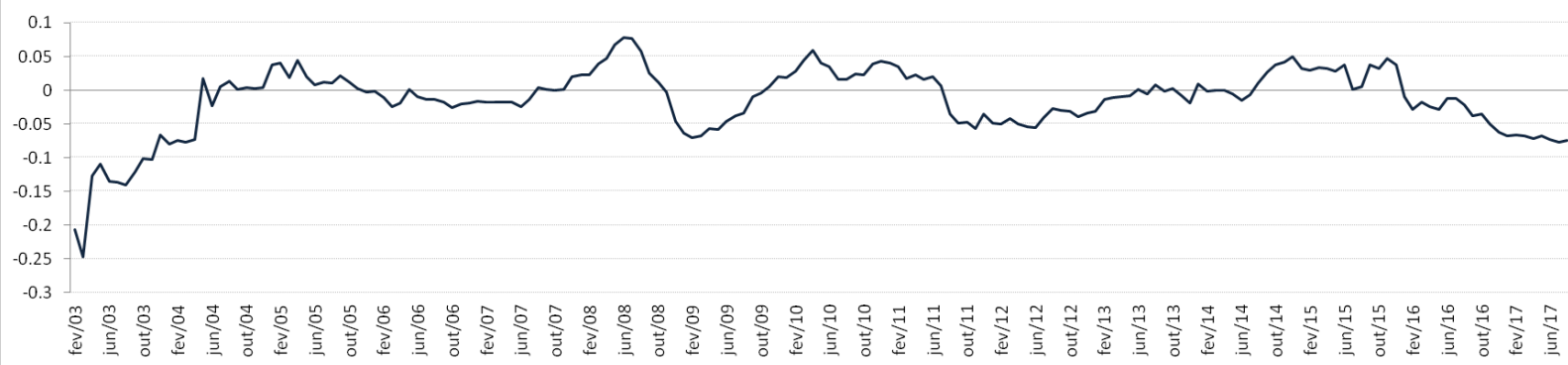

Também foi estimado o modelo com a incorporação de mudança de regime na persistência (MDNS-P), no qual faz-se a seguinte alteração no MDNS:

$$
\beta_{2 t}=\mu_{20}+\phi_{s t}\left(\beta_{2 t-1}-\mu_{20}\right)
$$

em que $\mathrm{s}=1,2$, conforme o regime. 
Figura 6 - Gráfico Variáveis Latentes MDNS-P

Beta $_{1 \mathrm{t}}$

Fev/2003 - Ago/2017

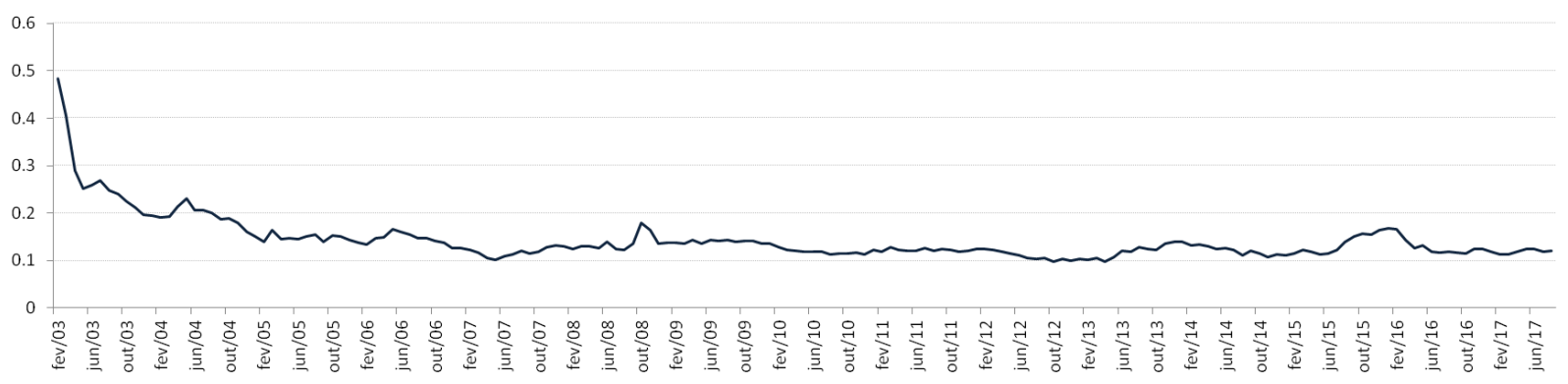

Beta $_{2 t}$

Fev/2003 - Ago/2017

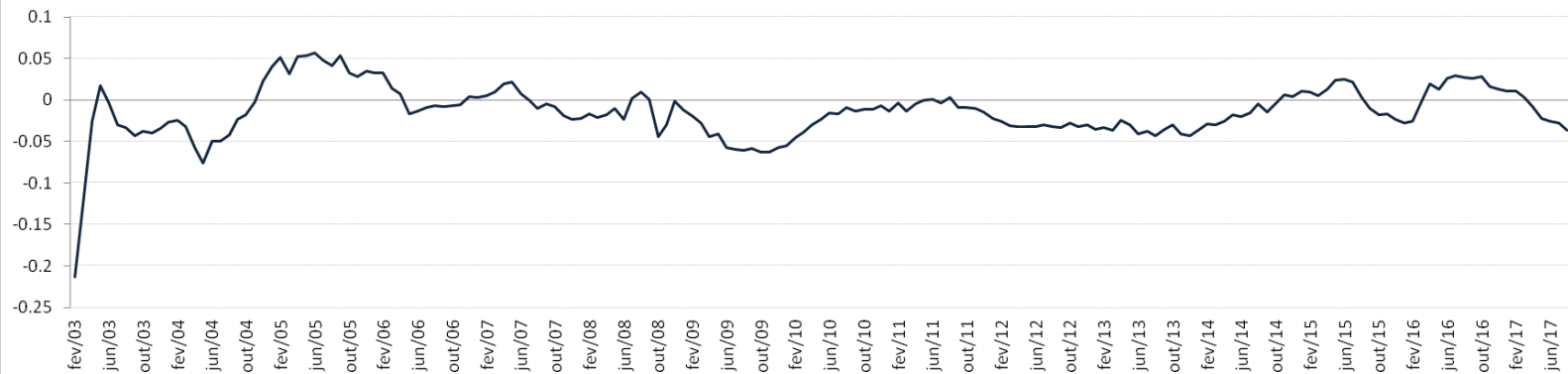

Beta $_{3 t}$

$\mathrm{Fev} / 2003$ - Ago/2017

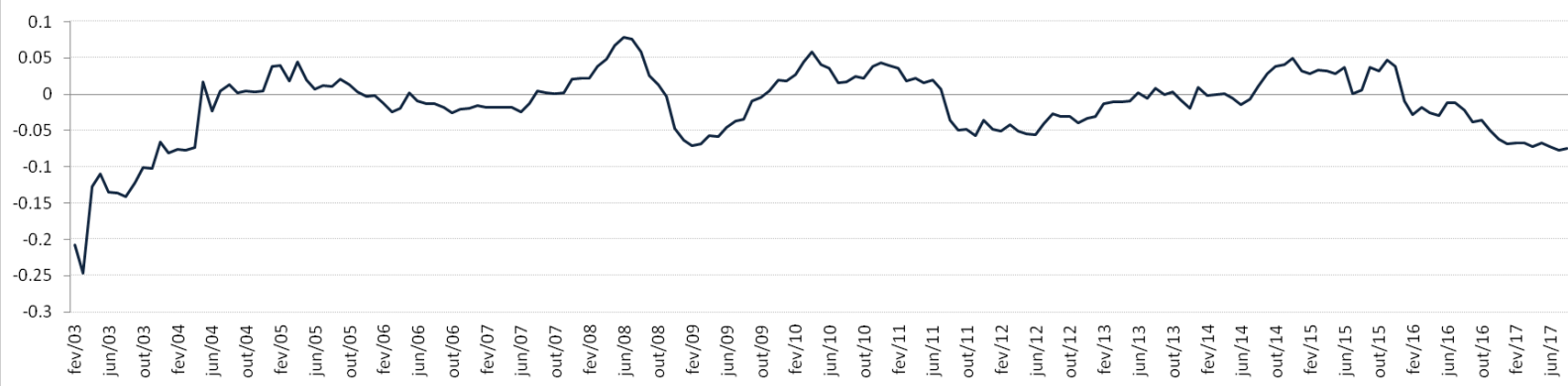

Nota-se também, pelo gráfico 17, que tanto no MDNS-M quanto no MDNS-P, as variáveis latentes estimadas são bastante similares às obtidas no MDNS. Com base na maioria dos critérios de escolha, baseados na análise do erro, tabela 2, o modelo MDNS ainda apresenta melhor ajuste na maturidade de três meses.

Além de considerarmos o fator de decaimento variante no tempo, dada a maior instabilidade política e econômica de países em desenvolvimento (e.g. Laurini e Hotta (2009)), foi estimado um MDNS com mudança de regime no $\lambda$ (MDNS- $\lambda$ ):

$$
y_{t}(\tau)=\beta_{1 t}+\beta_{2 t}\left(\frac{1-e^{-\lambda_{s t} \tau}}{\lambda_{s t} \tau}\right)+\beta_{3 t}\left(\frac{1-e^{-\lambda_{s t} \tau}}{\lambda_{s t} \tau}-e^{-\lambda_{s t} \tau}\right)
$$

em que s novamente representa o regime. 
Figura 7 - Gráfico Variáveis Latentes MDNS- $\lambda$

Beta $_{1 \mathrm{t}}$

Fev/2003- Ago/2017

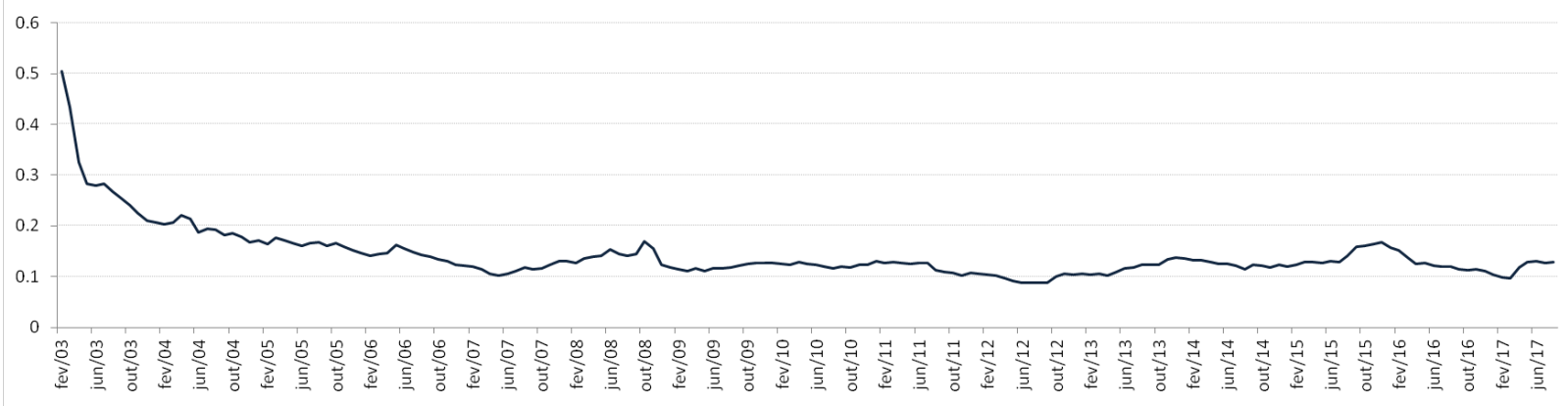

Beta $_{2 t}$

Fev/2003 - Ago/2017

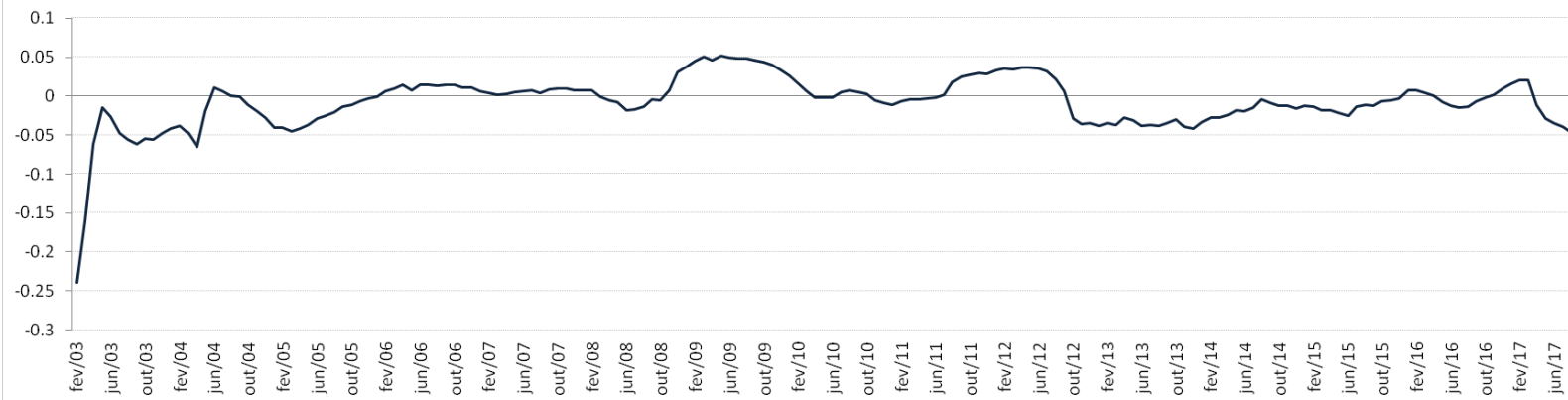

Beta $_{3 t}$

Fev/2003 - Ago/2017

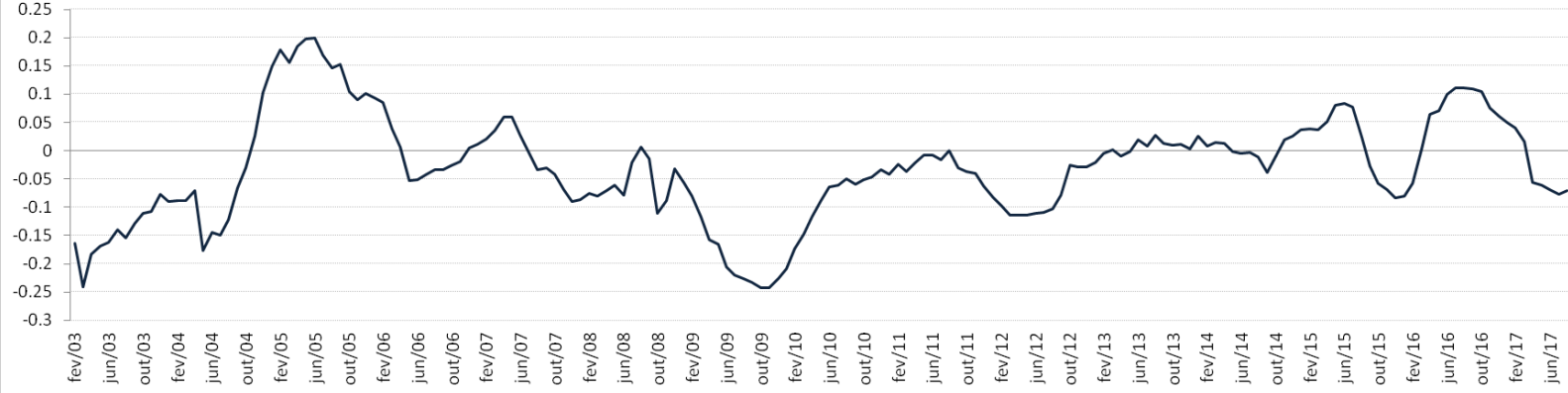

Neste modelo, as variáveis latentes de inclinação, $\beta_{2 t}$, e curvatura, $\beta_{3 t}$, possuem uma maior variação em relação aos modelos anteriores, podendo captar uma maior quantidade de eventos macroeconômicos, conforme veremos na comparação entre os modelos. Em relação aos erros de estimação, tabela 2, nota-se que este modelo apresenta melhor ajuste somente para a maturidade de 1 mês.

Agora, passamos a incorparação das variáveis macroeconômicas (MDNS-Macro). Neste modelo as variáveis são incorporadas de maneira exógena, ou seja, as variáveis macroeconômicas que afetam a curva de juros de maneira unidirecional. Os resultados são apresentados no gráfico 8: 
Figura 8 - Gráfico Variáveis Latentes MDNS-Macro
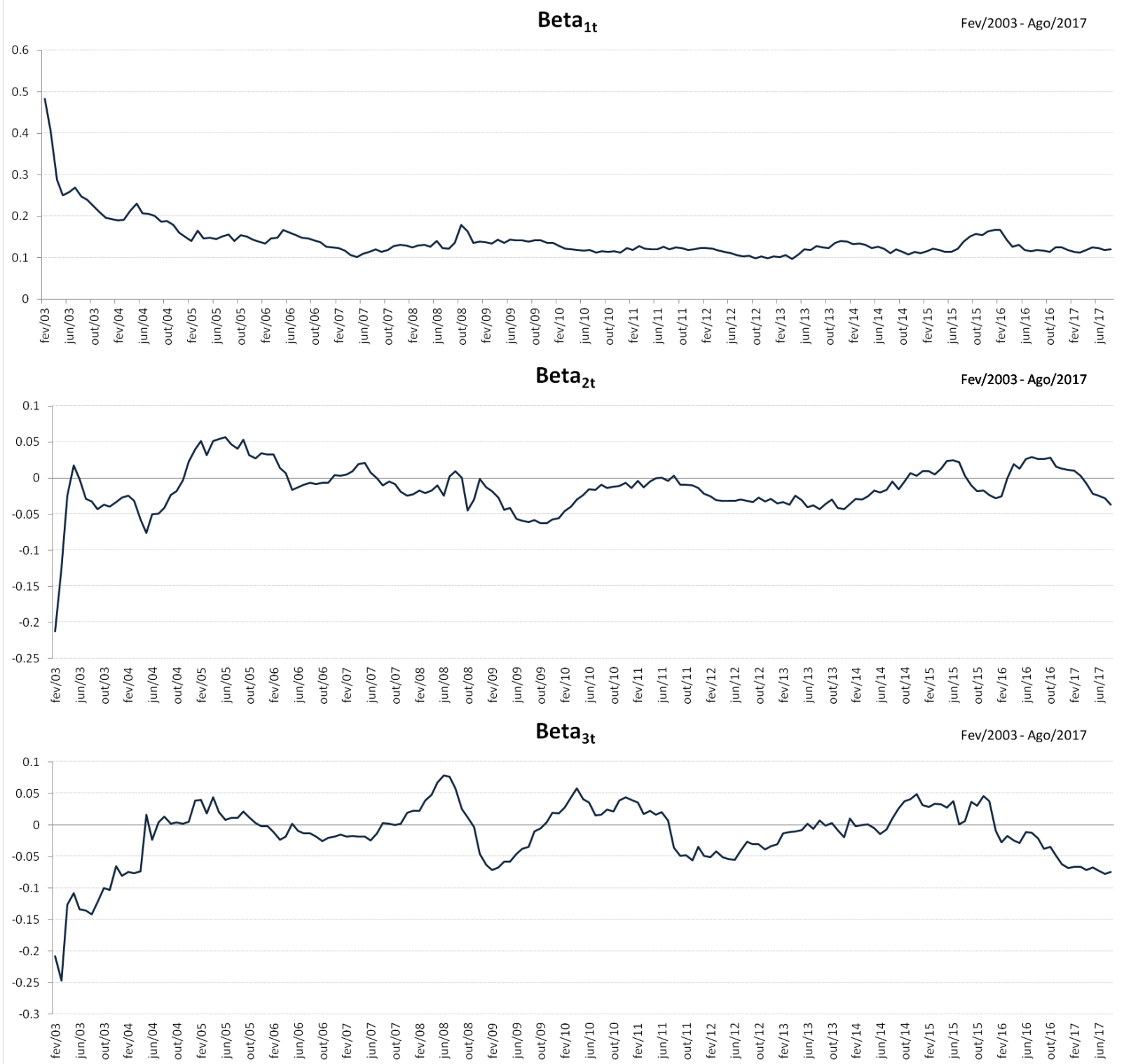

Incorporamos neste modelo (MDNS-Macro) também mudança de regime tanto na média (MDNS-MMacro), gráfico 9, quanto na persistência (MDNS-PMacro), gráfico 10. Estes modelos com incorporação de variáveis macroeconômicas apresentam melhor ajuste, de acordo com os critérios baseados nos erros da tabela 2, para maturidades de seis (MDNS-Macro), nove, doze e quinze meses (MDNS-PMacro). 
Figura 9 - Gráfico Variáveis Latentes MDNS-MMacro

Beta $_{1 \mathrm{t}}$

$\mathrm{Fev} / 2003$ - Ago/2017

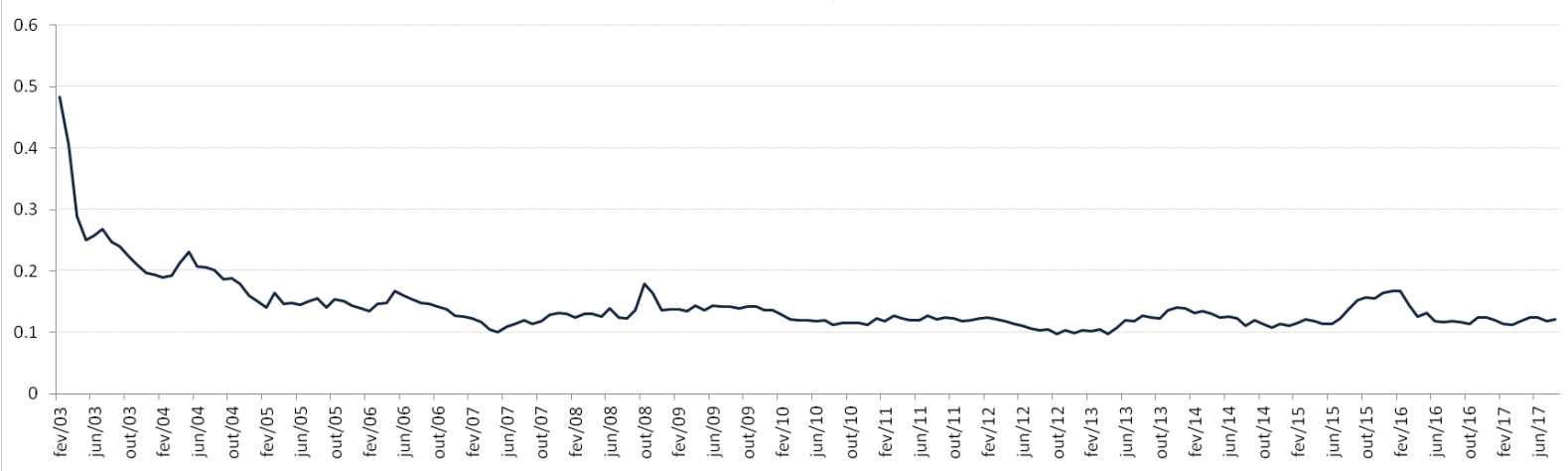

Beta $_{2 t}$

Fev/2003- Ago/2017

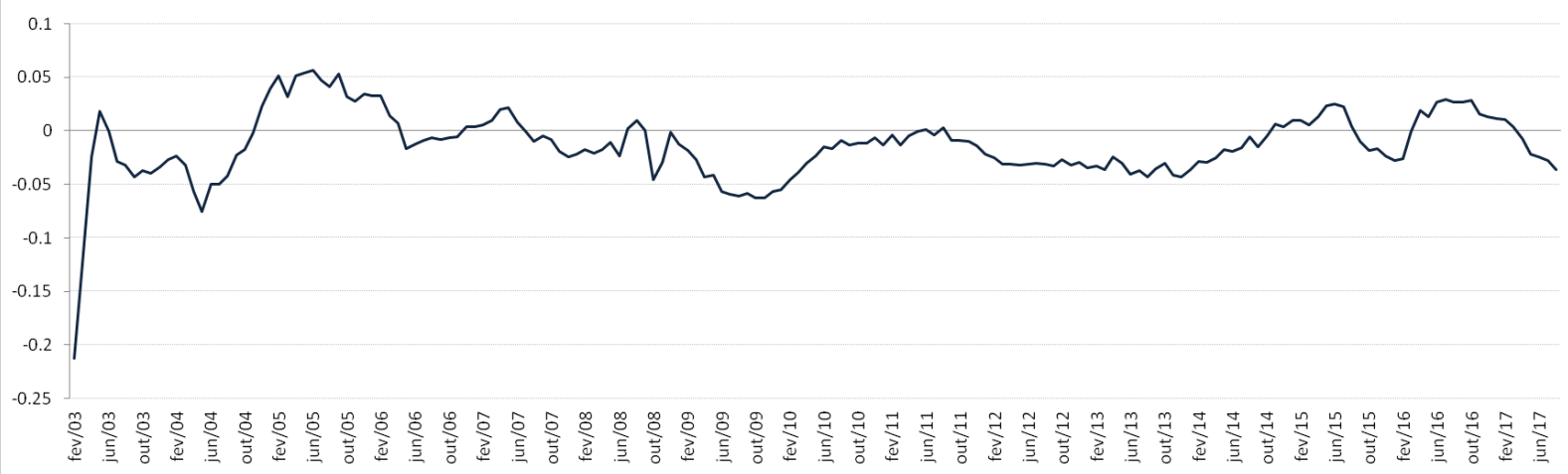

Beta $_{3 t}$

Fev/2003 - Ago/2017

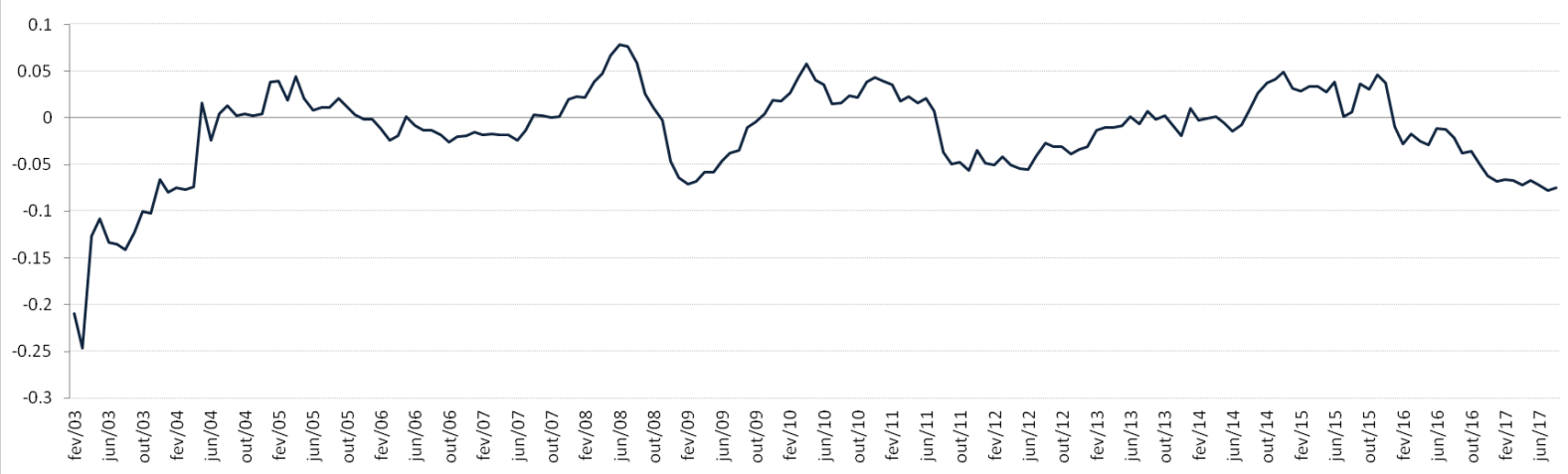


Figura 10 - Gráfico Variáveis Latentes MDNS-PMacro
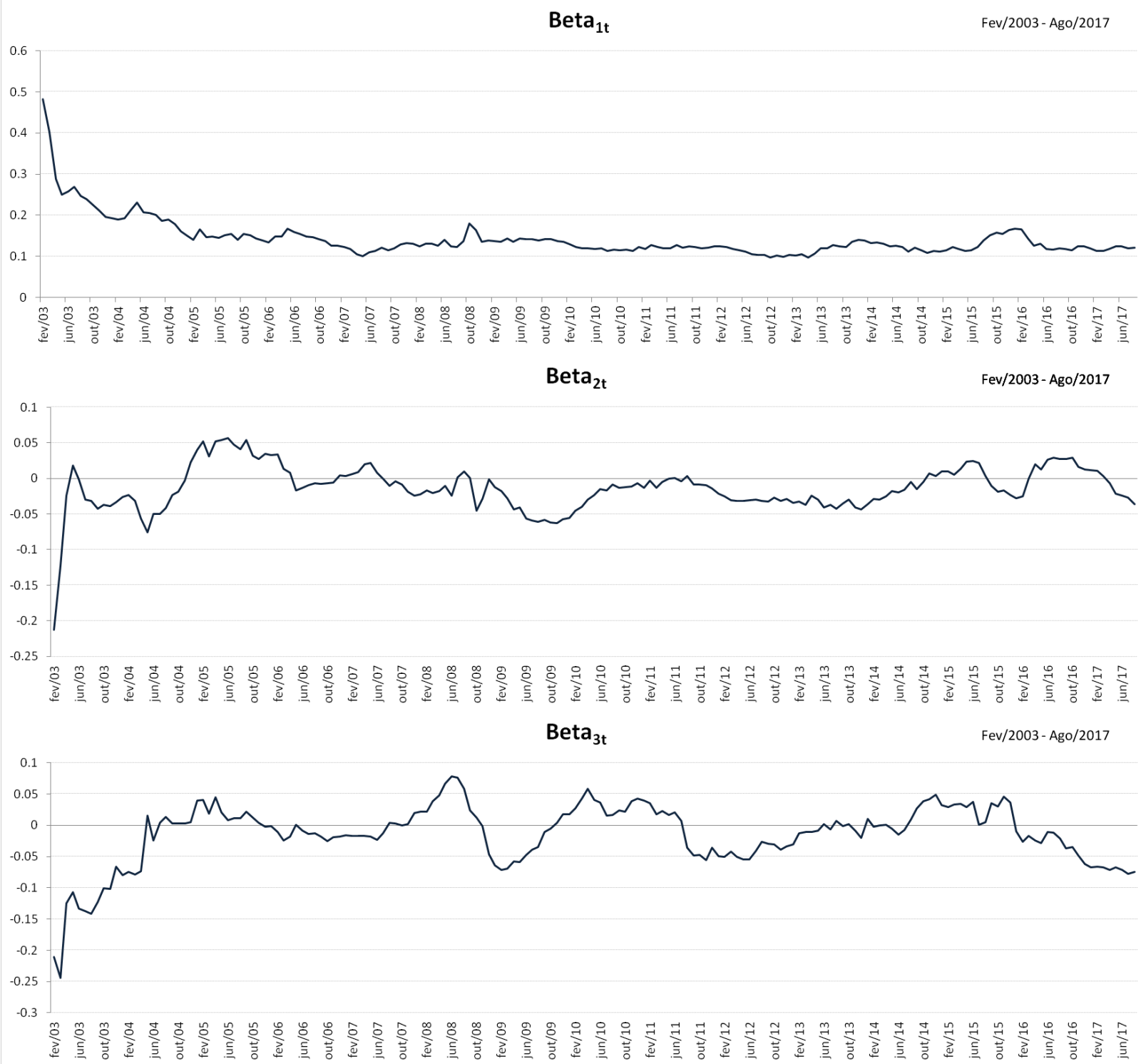

Até o momento, nos modelos com quebras foram fixados dois regimes, acrescentamos, então, a estimação do MDNS incorporando a mudança de regime especificada no texto de So et al. (1998) (MDNS-S). As variáveis latentes deste modelo são apresentadas no gráfico 11. Em relação ao modelo MDNS, estas variáveis apresentam pequenas oscilações. 
Figura 11 - Gráfico Variáveis Latentes MDNS-S

Beta $_{1 \mathrm{t}}$

$\mathrm{Fev} / 2003-\mathrm{Ago} / 2017$

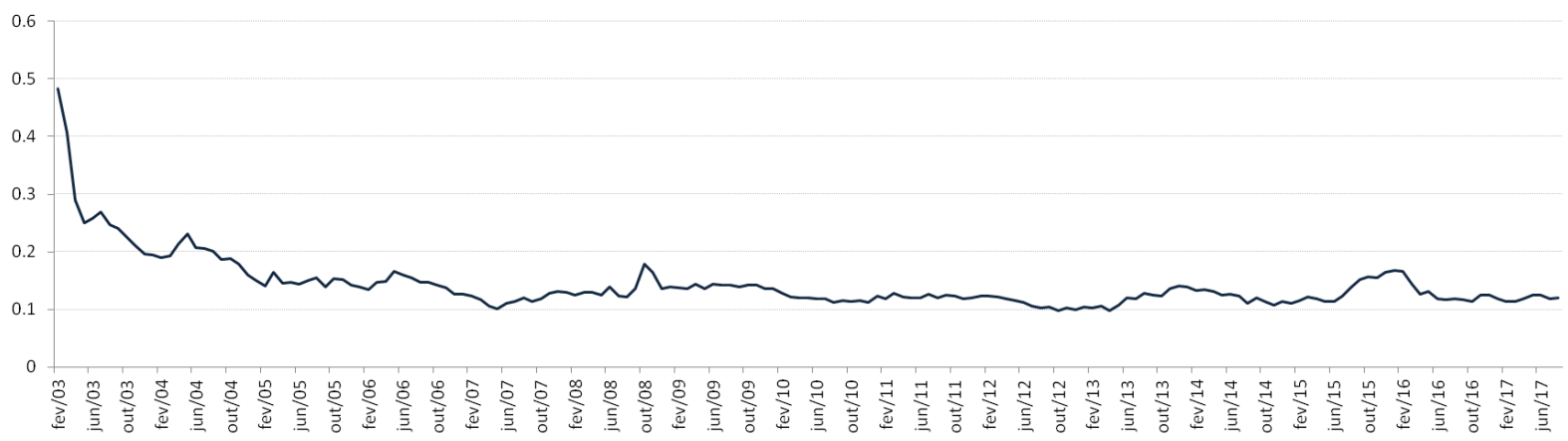

Beta $_{2 t}$

Fev/2003-Ago/2017

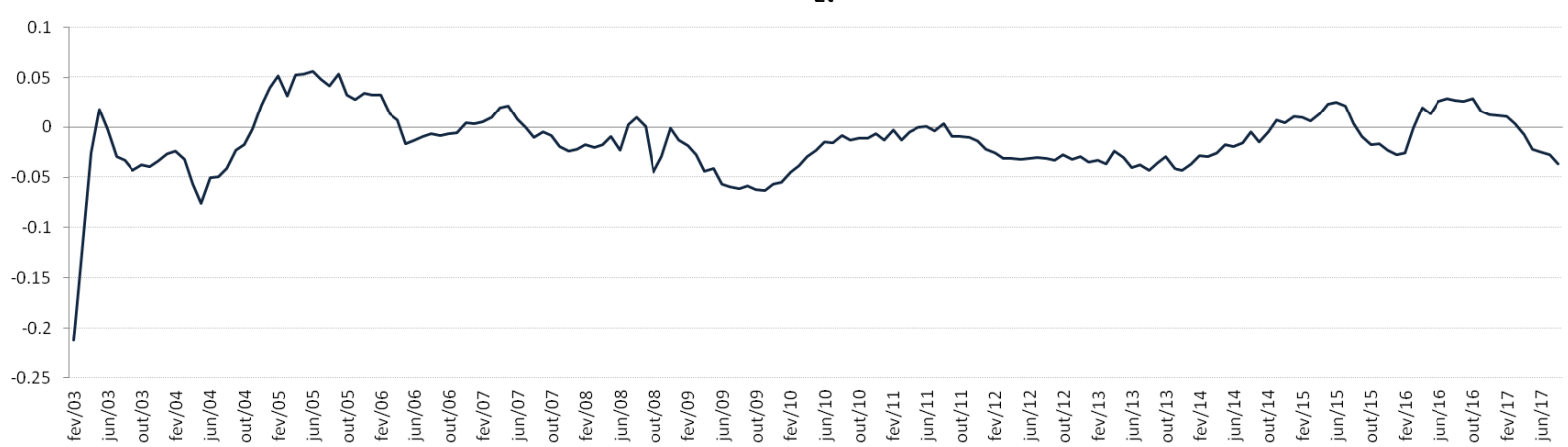

Beta $_{3 t}$

$\mathrm{Fev} / 2003$ - Ago/2017

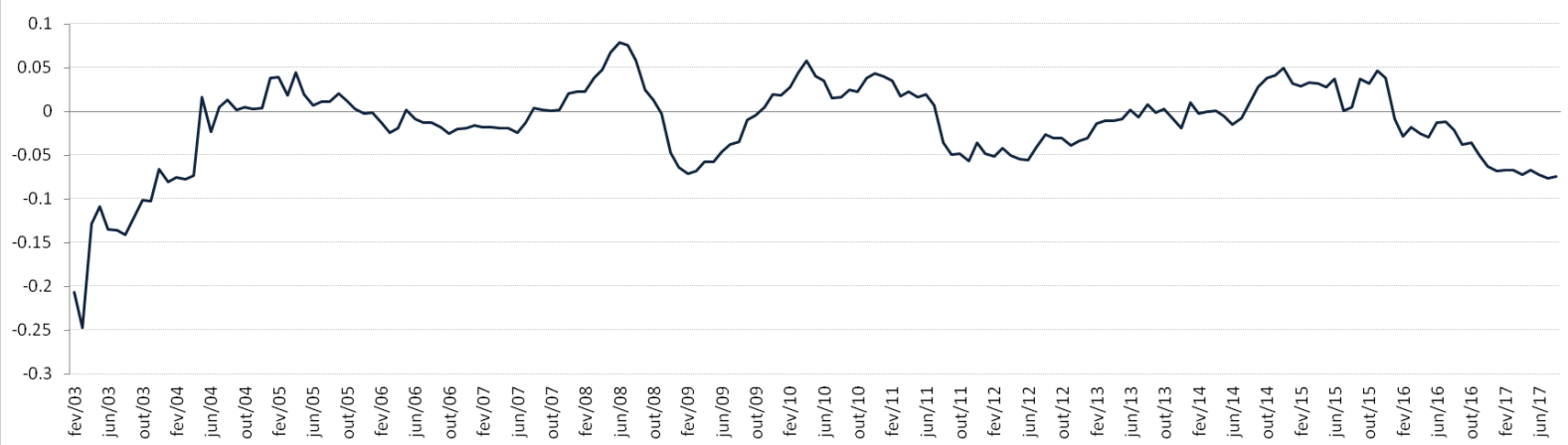

Também foram estimados modelos com a incorporação de variáveis macroeconômicas de maneira endógena e foram consideradas mudança de regime na variável latente e nas variáveis macroeconômicas. Os resultados do modelos com mudança de regime na média e nas variáveis macroeconômicas (MDNS-MMacroEnd) são apresentados no gráfico 12 : 
Figura 12 - Gráfico Variáveis Latentes MDNS-MMacroEnd

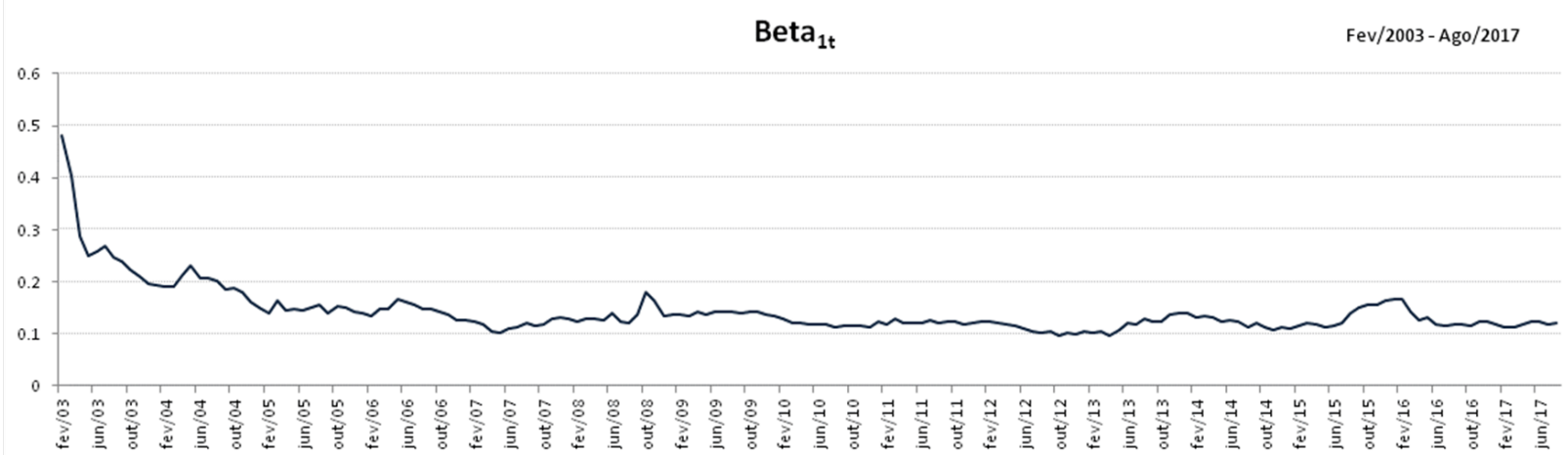

Beta $_{2 t}$

Fev/2003-Ago/2017

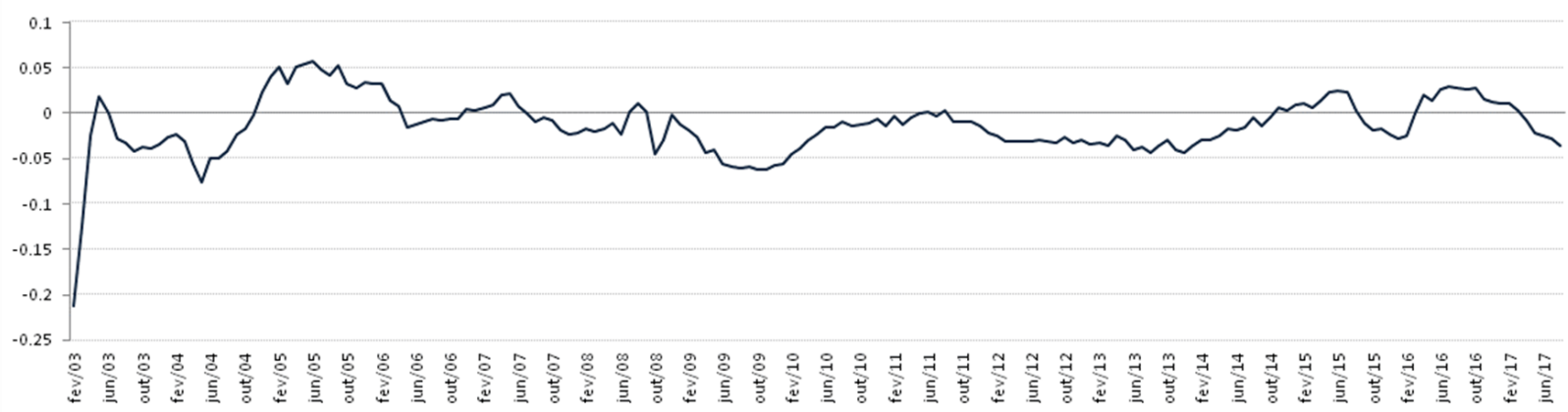

Beta $_{3 t}$

$\mathrm{Fev} / 2003$ - Ago/2017

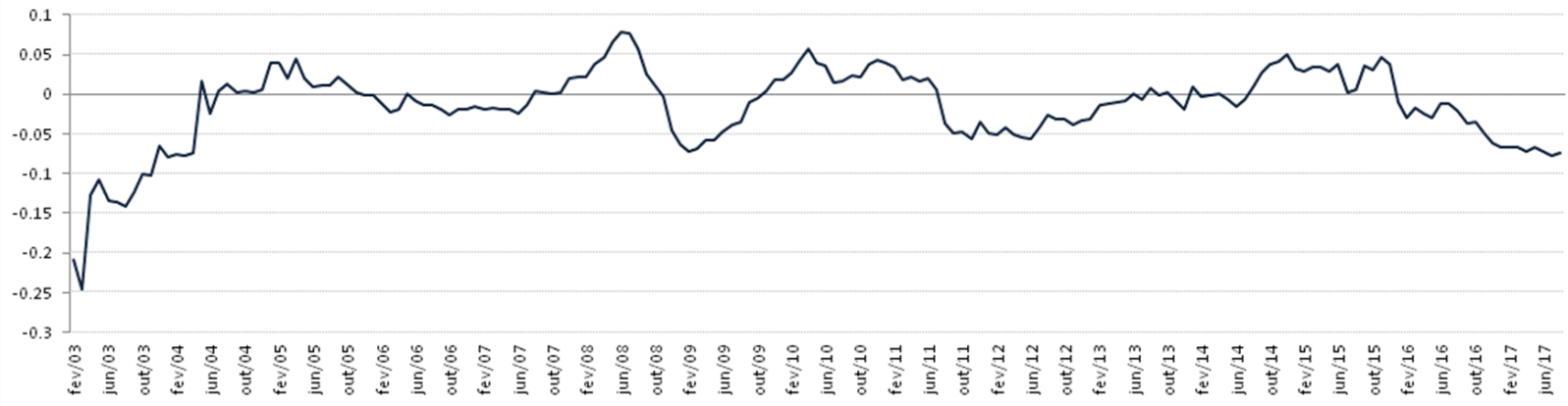

Já no modelo que incorpora mudança de regime na persistência e nas variáveis macroeconômicas (MDNS-PMacroEnd), os fatores são apresentados no gráfico 13: 
Figura 13 - Gráfico Variáveis Latentes MDNS-PMacroEnd

Beta $_{1 \mathrm{t}}$

Fev/2003-Ago/2017

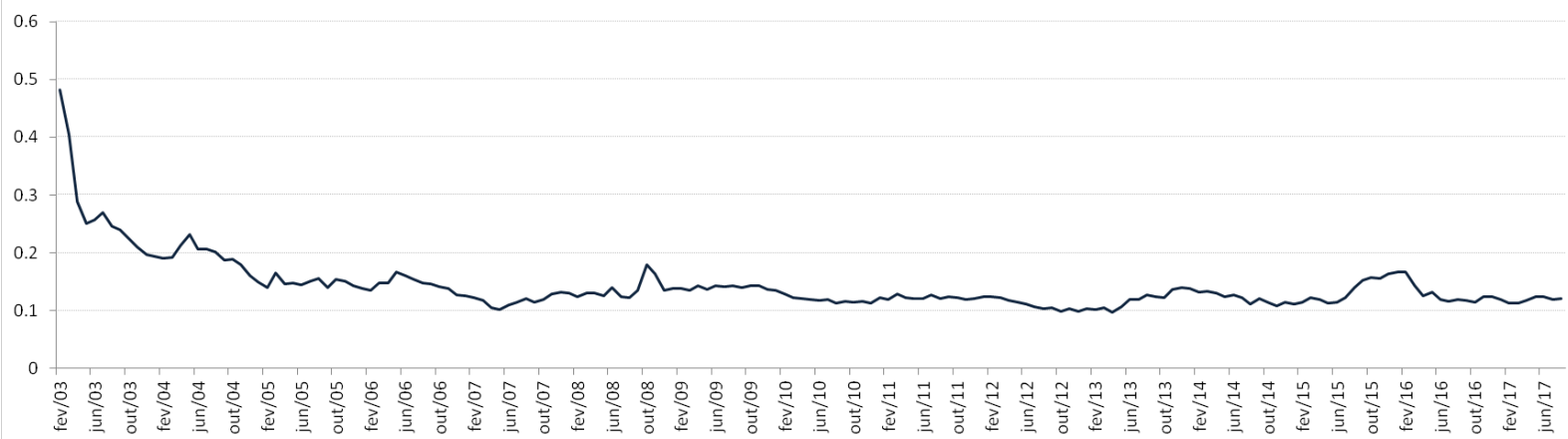

Beta $_{2 t}$

Fev/2003-Ago/2017

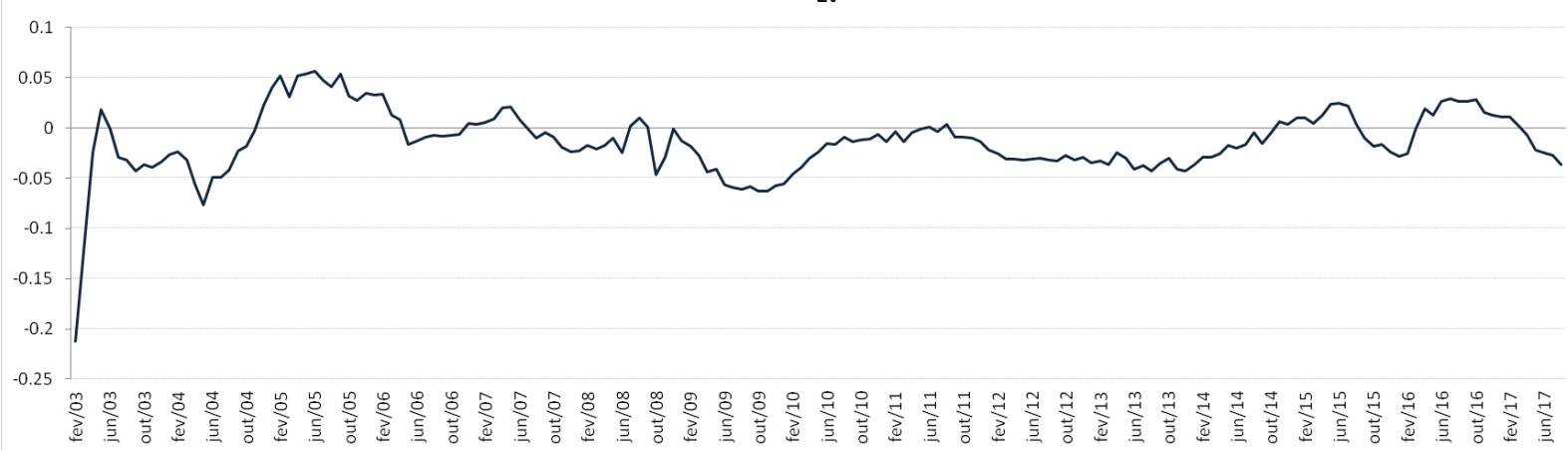

Beta $_{3 t}$

$\mathrm{Fev} / 2003$ - Ago/2017

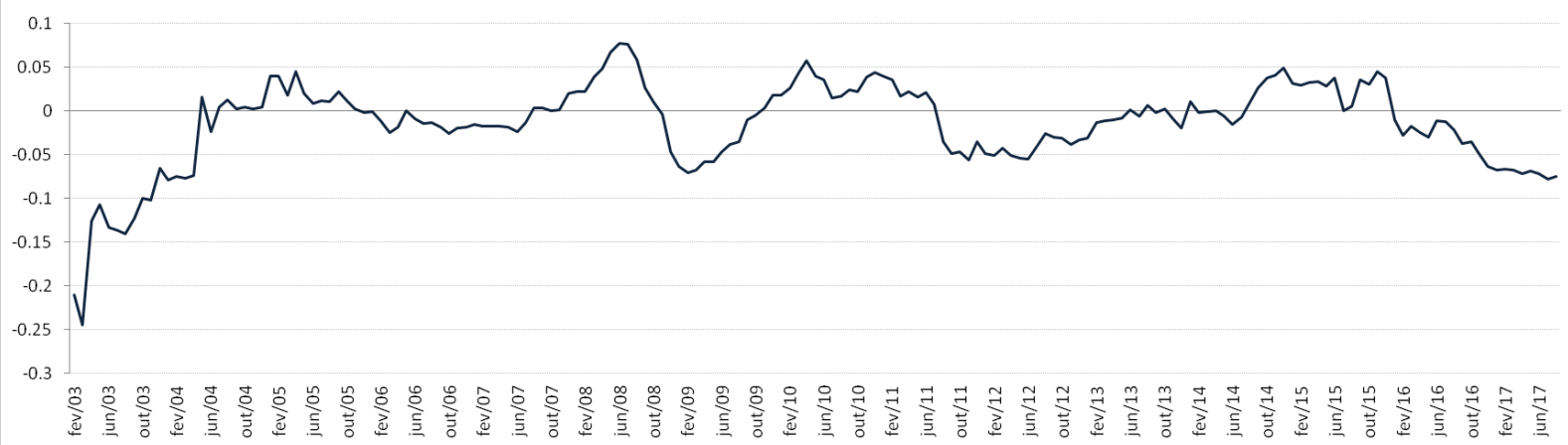

Também estimamos um modelo com a mudança de regime conforme So et al. (1998) no fator de decaimento (MDNS-S $\lambda$ ). Na figura 14, são apresentadas as variáveis latentes estimadas em comparação com as do modelo MDNS. 
Figura 14 - Gráfico Comparativo Variáveis Latentes MDNS e MDNS-S $\lambda$

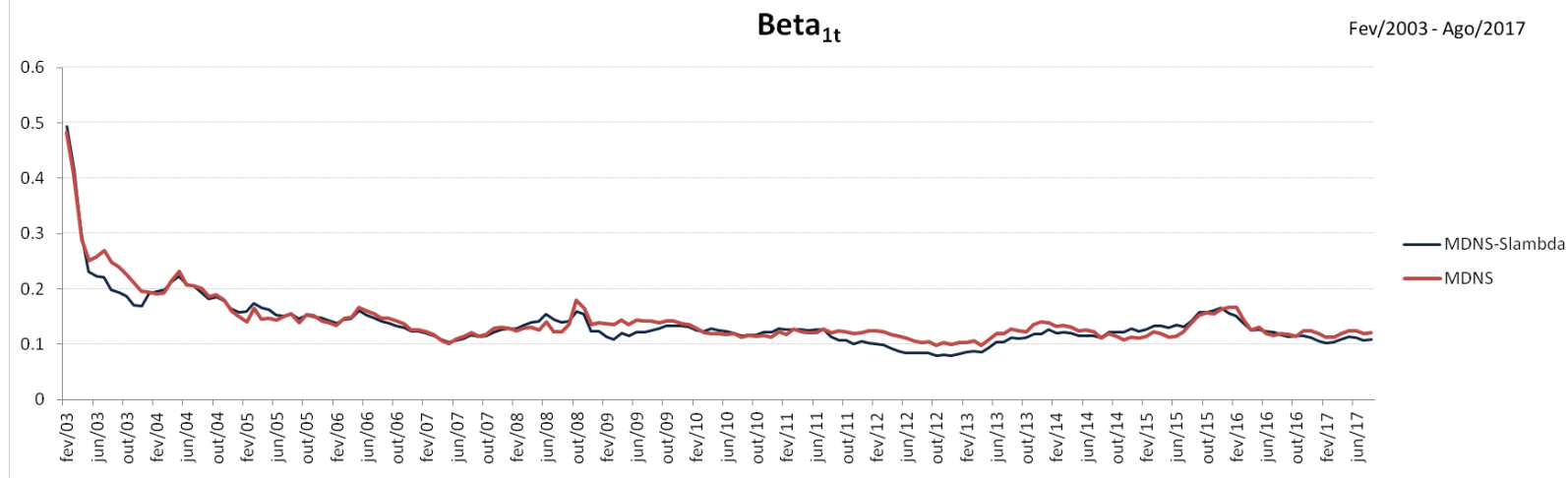

Beta $_{2 \mathrm{t}}$

Fev/2003 - Ago/2017
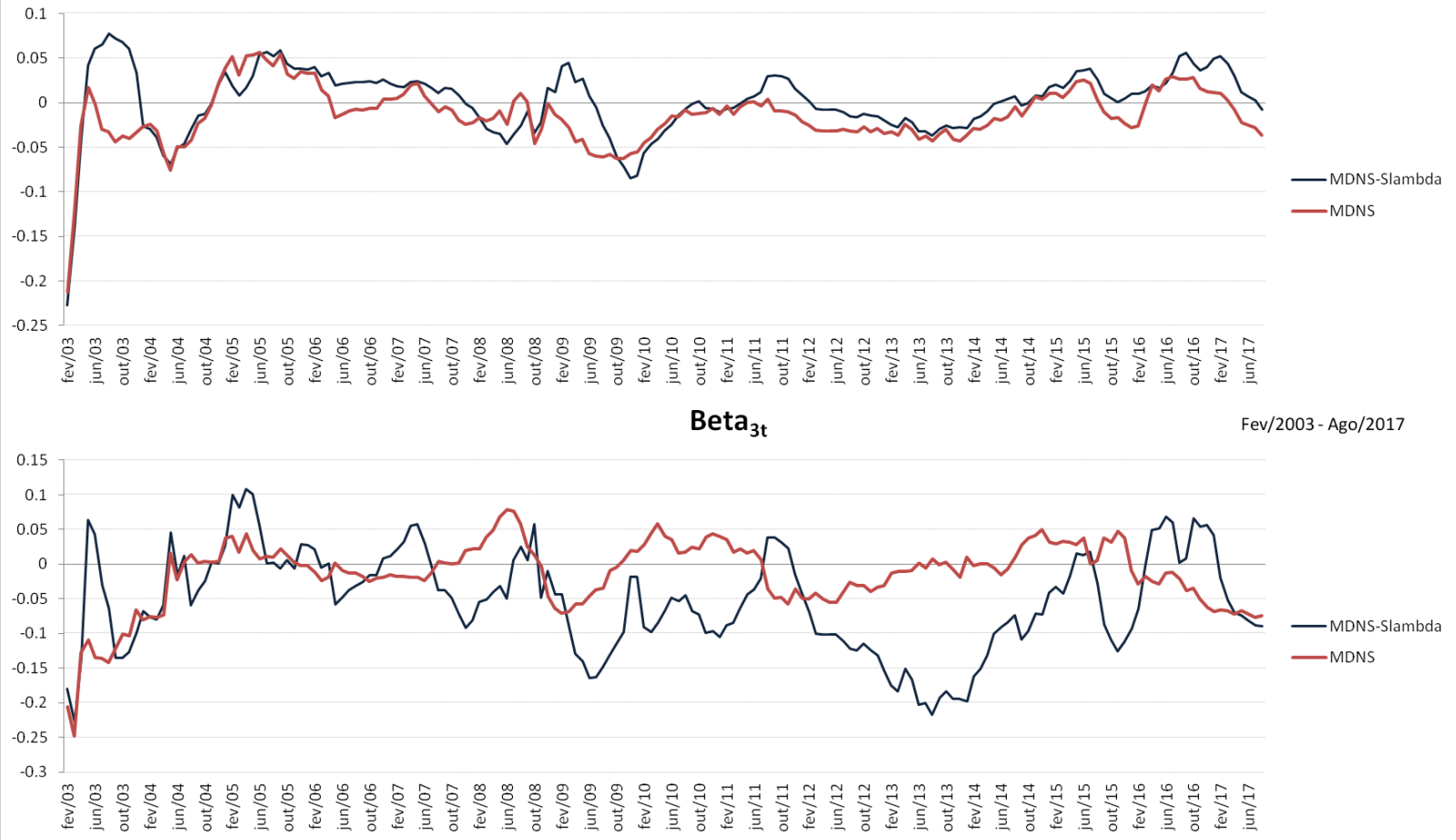

Assim, como em MDNS- $\lambda$, este modelo apresenta maior oscillação em relação ao MDNS. Os modelos MDNS-SmediaMacro e MDNS-Smacro também apresentam oscilações na inclinação e curvatura em relação às variáveis latentes estimadas do MDNS, no entanto menos acentuadas, conforme gráficos 15 e 16. 
Figura 15 - Gráfico Comparativo Variáveis Latentes MDNS e MDNS-SmediaMacro

Beta $_{1 \mathrm{t}}$

Fev/2003 - Ago/2017

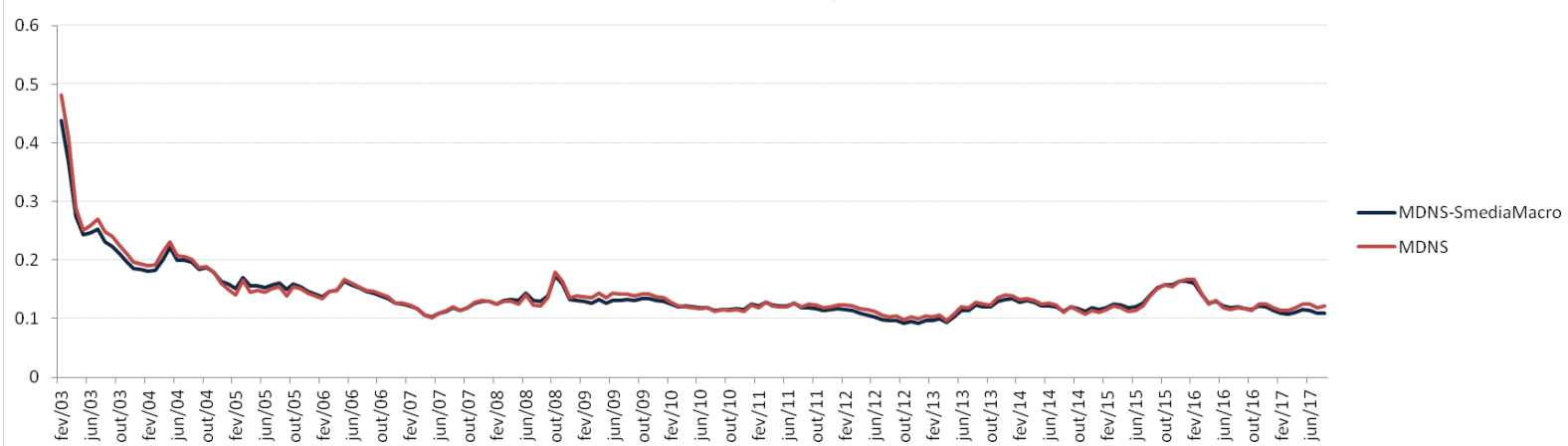

Beta $_{2 t}$

Fev/2003-Ago/2017

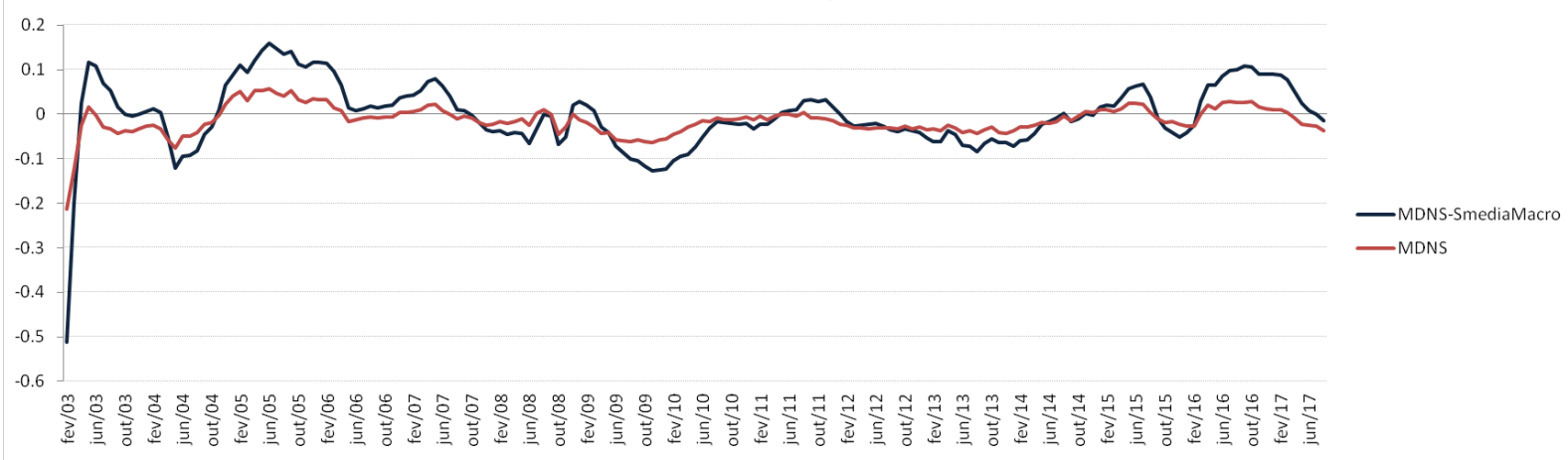

Beta $_{3 t}$

$\mathrm{Fev} / 2003-\mathrm{Ago} / 2017$

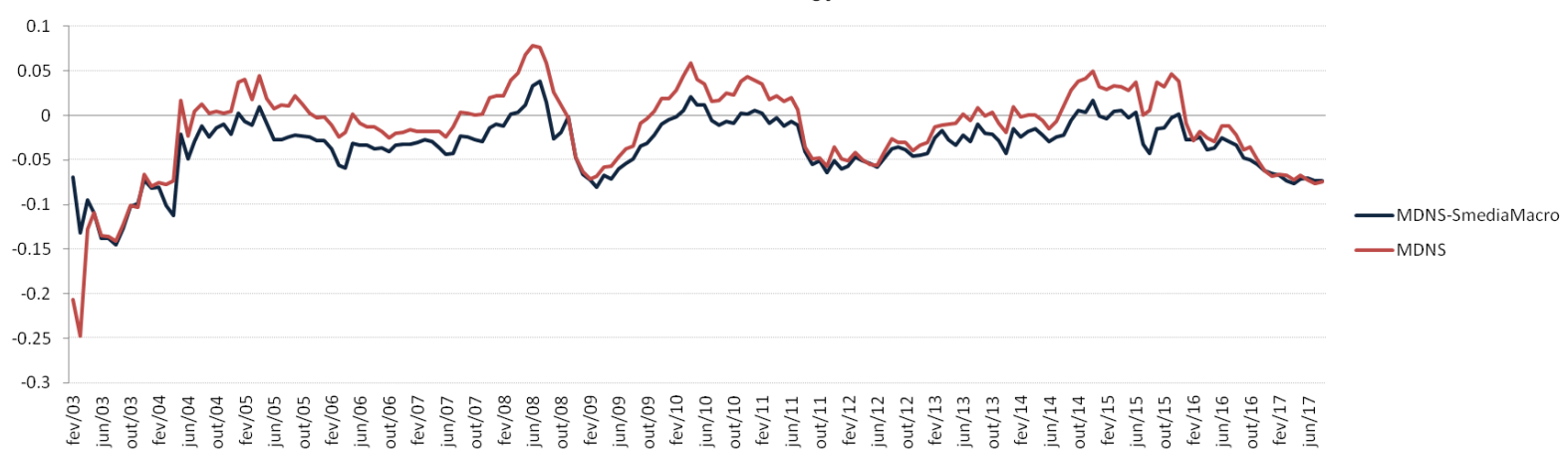


Figura 16 - Gráfico Comparativo Variáveis Latentes MDNS e MDNS-Smacro
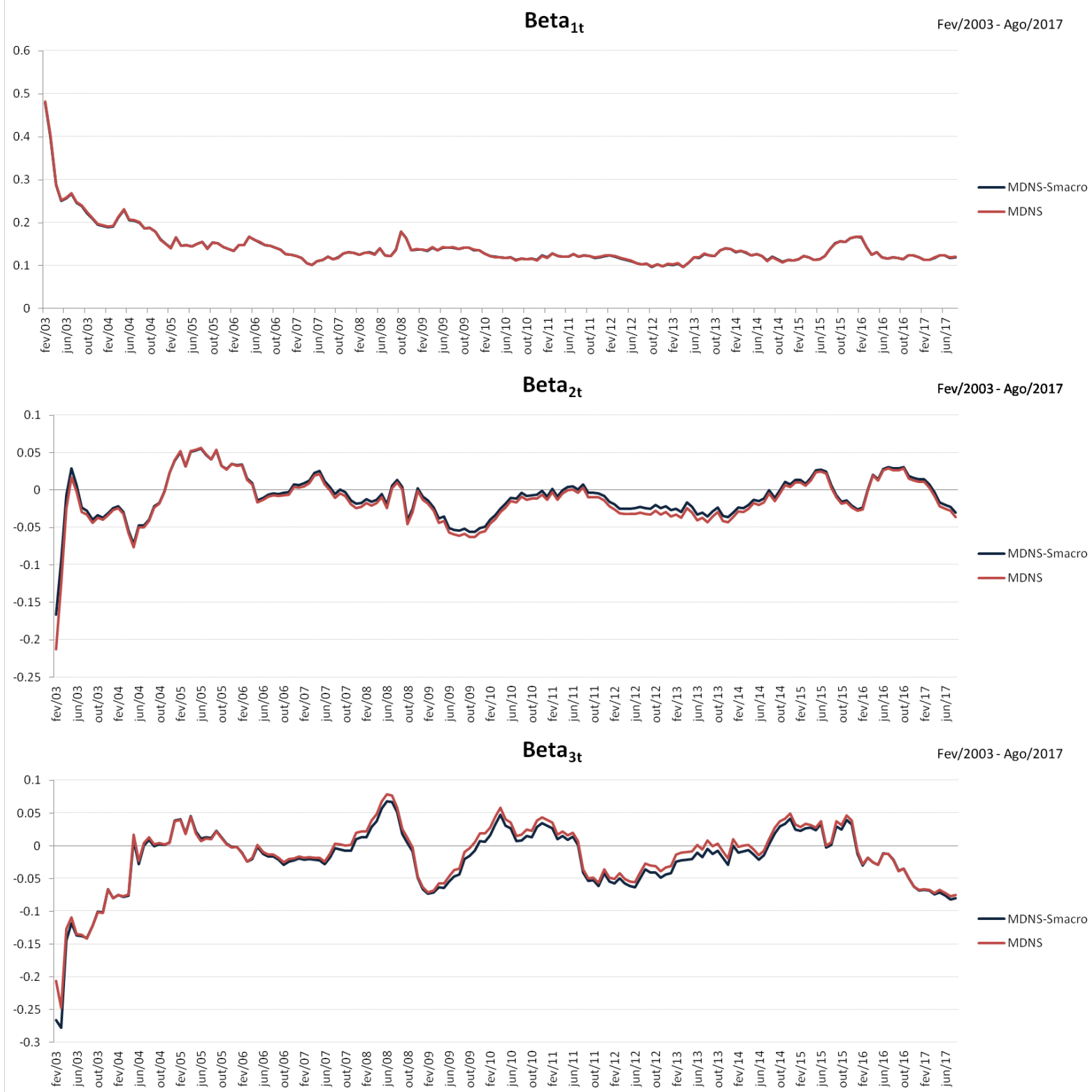

A média dos parâmetros estimados de $\beta_{1 t}, \beta_{2 t}$ e $\beta_{3 t}$, em cada modelo analisado, estão apresentadas no gráfico 17. As variáveis latentes de nível, $\beta_{1 t}$, são muito similares em todos os modelos aqui estimados. O parâmetro de inclinação e curvatura já apresentam divergências entre os modelos, sendo que os três primeiros modelos (MDNS, MDNS-M, MDNS-P) apresentam fatores bastante similares. Os modelos em que estes parâmetros apresentam um descolocamento maior são MDNS- $\lambda$ e o MDNS-S $\lambda$, ou seja, os modelos em que a mudança de regime é no fator de decaimento.

As oscilações dos fatores latentes podem ser associadas a acontecimentos macroeconômicos, tais como mudanças de política econômica ou fiscal, incertezas em relação ao cenário político e ao cenário internacional. 
Com isso, nota-se que os fatores latentes de todos os modelos estimados capturam a crise de expectativas na corrida eleitoral de 2002, que persiste no início do governo petista em 2003.

Com o pós-crise das eleições, o fator de inclinação, componente de curto prazo da curva de juros, exibiu inversão em seu comportamento, mostrando que a curva estava adquirindo uma forma mais linearizada e, ao mesmo tempo, reiterando o aumento das cargas verificadas pelos fatores de inclinação.

Posteriormente, o nível das curvas de juros, $\beta_{1 t}$, de todos os modelos se torna mais estável, sendo que demais acontecimentos macroeconômicos são capturados pelos fatores latentes de inclinação, $\beta_{2 t}$, e curvatura, $\beta_{3 t}$, conforme veremos a seguir.

Em junho 2004, nota-se uma queda de $\beta_{2 t}$ devido à perspectiva de aumento na taxa de juros nos EUA, que vinham de um longo período de queda desde 11 de setembro, o que levaria para aquele país recursos até então aplicados no Brasil. Como o aumento efetivo é pouco significativo, esta queda da inclinação logo se reverte. Em todos os modelos a inclinação captura este evento, no entanto no modelo MDNS-SmediaMacro esta queda é mais acentuada.

Os fatores latentes de inclinação e curvatura capturam o período pós-crise no início de 2009. Entre 2008 e 2009, nota-se uma queda da curvatura, devido a crise internacional desencadeada inicialmente no mercado imobiliário norte-americano. Isto levou a saída de dinheiro do mercado brasileiro, e o aumento da inflação no Brasil. Os modelos com mudança de regime no fator de decaimento apresentam uma queda mais acentuada na curvatura em um período um pouco posterior, já os demais modelos captam esta queda de maneira menos acentuada, no entanto mais concentrada no final de 2008 e início de 2009.

Em 2011 e 2012, houve uma elevação da inflação desde 2008. de acordo com o Boletim Focus. Evento captutado essencialmente pelo modelo MDNS-SmediaMacro na inclinação e pelo modelo MDNS-S $\lambda$ na curvatura.

Ademais, nota-se uma oscilação na curvatura, essencialmente nos modelos com mudança de regime no decaimento, no período de junho de 2014 a agosto de 2017. Isto se deu devido a crise desencadeada em 2014, que de acordo com Filho (2017), teve origem em uma série de choques de oferta e demanda, na maior parte ocasionados por erros de políticas públicas que reduziram a capacidade de crescimento da economia brasileira e geraram um custo fiscal elevado, além de ser agravada pela crise política.

Com isso, nota-se que os fatores médios do MDNS- $\lambda$ e, principalmente, do MDNSS $\lambda$ e MDNS-SmediaMacro são capazes de identificar os acontecimentos macroeconômicos de forma mais consistente. 
Figura 17 - Gráfico Comparativo das Variáveis Latentes

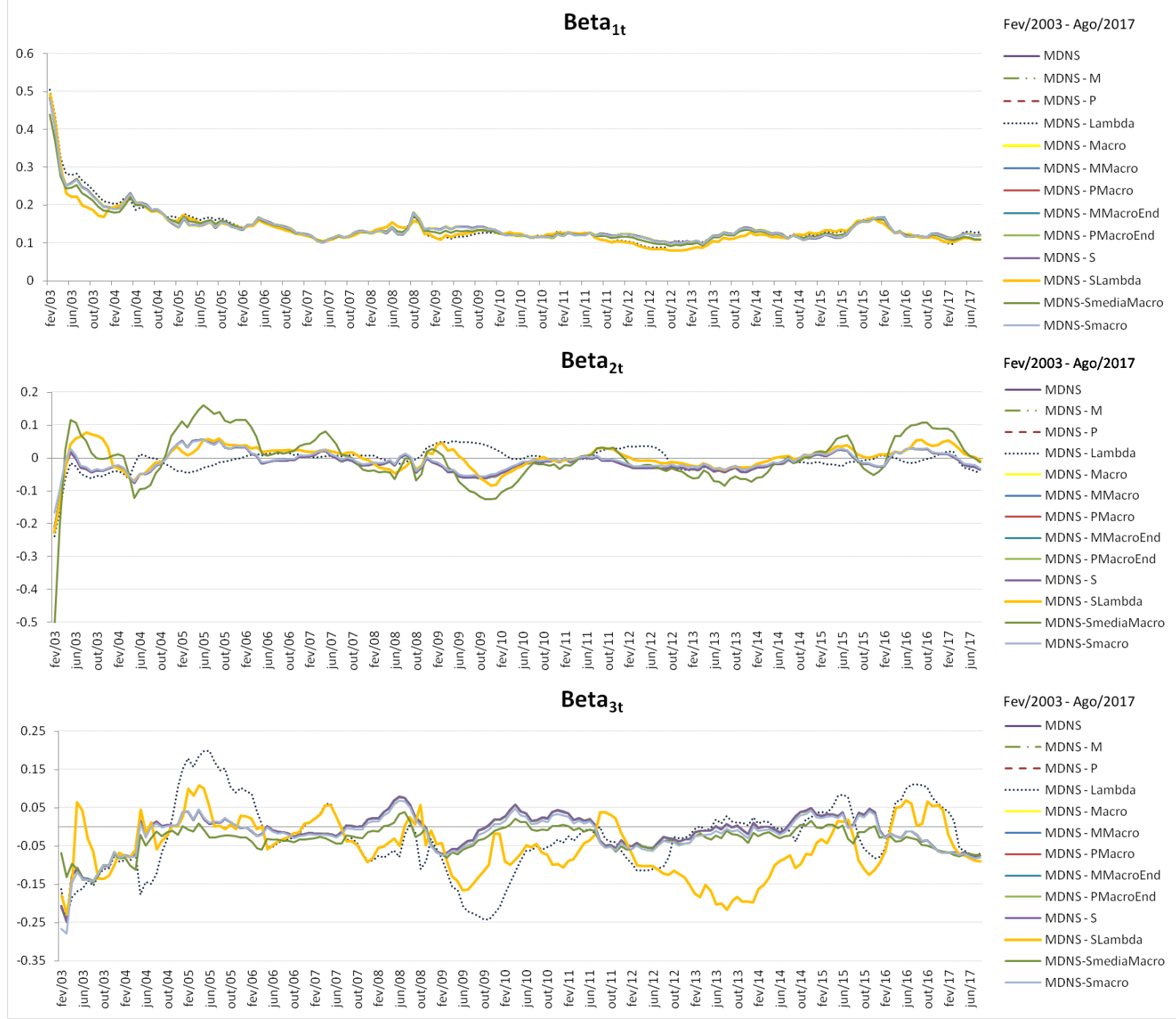

Acima foram apresentadas as médias das variáveis latentes da curva de juros em relação às maturidades em cada período aqui analisado. Para uma comparação mais direta e detalhada entre os modelos, foram analisados os critérios de escolhas, ME (Mean Error), RMSE (Root Mean Squared Error) e MAE (Mean Absolute Error), em cada maturidade, os quais são apresentados na tabela 2. Na maturidade de um mês, o modelo com melhor performance nos três critérios de escolha foi o modelo dinâmico de Nelson-Siegel com mudança de regime no fator de decaimento (MDNS- $\lambda$ ). Para as maturidades de 6 a 48 meses, nota-se que a incorporação de variáveis macroeconômicas se mostrou relevante para um melhor ajuste, com destaque para os modelos que incorporam mudança de regime na persistência (modelos MDNS-PMacro e MDNS-PMacroEnd). Além disso, modelos mais flexíveis, MDNS-PMacroEnd e MDNS-SMacro, possuem melhor ajuste na maioria nas maturidades superiores a 15 meses. 
Tabela 2 - Erros de Estimação

\begin{tabular}{|c|c|c|c|c|c|c|c|c|c|c|c|c|c|}
\hline \multicolumn{14}{|c|}{ Erro Médio } \\
\hline & MDNS & $\begin{array}{c}\text { MDNS- } \\
\text { M }\end{array}$ & $\begin{array}{l}\text { MDNS- } \\
\quad \text { P }\end{array}$ & $\begin{array}{c}\text { MDNS- } \\
\quad \lambda\end{array}$ & $\begin{array}{l}\text { MDNS- } \\
\text { Macro }\end{array}$ & $\begin{array}{l}\text { MDNS- } \\
\text { MMacro }\end{array}$ & $\begin{array}{l}\text { MDNS- } \\
\text { PMacro }\end{array}$ & $\begin{array}{l}\text { MDNS- } \\
\text { MMacro } \\
\text { End }\end{array}$ & $\begin{array}{l}\text { MDNS- } \\
\text { PMacro } \\
\text { End }\end{array}$ & $\begin{array}{c}\text { MDNS- } \\
\mathrm{S}\end{array}$ & $\begin{array}{l}\text { MDNS- } \\
\text { SLambda }\end{array}$ & $\begin{array}{l}\text { MDNS- } \\
\text { Smedia } \\
\text { Macro }\end{array}$ & $\begin{array}{l}\text { MDNS- } \\
\text { Smacro }\end{array}$ \\
\hline $1 \mathrm{M}$ & 0,000367 & 0,000364 & 0,000368 & 0,000304 & 0,000357 & 0,000356 & 0,000352 & 0,000344 & 0,000353 & 0,000368 & 0,001133 & 0,000840 & 0,000314 \\
\hline $3 \mathrm{M}$ & 0,000030 & 0,000029 & & 0,000898 & 0,000028 & 0,000029 & 0,000027 & 0,000021 & & & $-0,0$ & $-0,000713$ & \\
\hline $6 \mathrm{M}$ & $-0,000245$ & $-0,000243$ & $-0,000244$ & $-0,000529$ & $-0,000239$ & $-0,000238$ & $-0,000237$ & $-0,000241$ & $-0,000237$ & $-0,000244$ & $-0,002045$ & $-0,001227$ & $-0,000246$ \\
\hline $9 \mathrm{M}$ & $-0,000398$ & $-0,000395$ & $-0,000397$ & $-0,001109$ & $-0,000390$ & $-0,000389$ & $-0,000386$ & $-0,000389$ & $-0,000387$ & $-0,000$ & 251 & $-0,00$ & $-0,000443$ \\
\hline $12 \mathrm{M}$ & $-0,000300$ & $-0,000298$ & $-0,000300$ & $-0,001070$ & $-0,000293$ & $-0,000292$ & $-0,000290$ & $-0,000291$ & $-0,000290$ & $-0,000299$ & $-0,001983$ & $-0,001157$ & $-0,000359$ \\
\hline $15 \mathrm{M}$ & & $-0,00$ & & $-0,000799$ & $-0,000113$ & $-0,000113$ & $-0,0$ & & & & & 0833 & $-0,000180$ \\
\hline $18 \mathrm{M}$ & 0,000037 & 0,000038 & 0,000037 & $-0,000495$ & 0,000040 & 0,000040 & 0,000041 & 0,000 & 0,000041 & 037 & $-0,0$ & $-0,000516$ & $-0,000020$ \\
\hline $21 \mathrm{M}$ & & & & $-0,000239$ & 0,000128 & 0,000128 & & & & & & & 0,000079 \\
\hline $24 \mathrm{M}$ & 0,000218 & 000219 & 0,000218 & 0,000017 & 0,000217 & 0,000217 & 0,000 & 0,000218 & 0,000217 & 0,000219 & $-0,000229$ & 0,000004 & 0,000180 \\
\hline $27 \mathrm{M}$ & 0,000236 & 0,000235 & 0,000235 & 0,000187 & 0,000233 & 0,000232 & 0,000231 & 0,000232 & 0,000231 & 0,000236 & 0,000114 & 0,000187 & 0,000209 \\
\hline $30 \mathrm{M}$ & 0,000184 & 0,000183 & 0,000184 & 0,000272 & 0,000180 & 0,000179 & 0,000178 & 0,000179 & 0,0001 & 0,000184 & 0,000376 & 0,000295 & 0,000169 \\
\hline $33 \mathrm{M}$ & 0,000123 & 0,000121 & 0,000122 & 0,000329 & 0,000118 & 0,000117 & 0,000115 & 0,000117 & 0,000116 & 0,000122 & 0,000612 & 0,000386 & 0,000119 \\
\hline $36 \mathrm{M}$ & 0,000088 & & & & & 0,000082 & & & & & & 0,000495 & 0,000097 \\
\hline $39 N$ & 0,000104 & 0,000103 & 0,000104 & 0,000 & 0,000099 & 0,000098 & 0,000097 & 0,000097 & 0,0000 & 0,000103 & 0,001137 & 0,000646 & 0,000124 \\
\hline $48 \mathrm{~N}$ & & & & 0,000507 & $-0,000049$ & $-0,000049$ & $-0,000050$ & $-0,000051$ & $-0,000050$ & $-0,000047$ & & 0,000846 & 0,000005 \\
\hline $60 \mathrm{~N}$ & & & & & $-0,000194$ & & $-0,000192$ & $-0,000195$ & $-0,0001$ & $-0,000196$ & & 0,001057 & $-0,000110$ \\
\hline $72 \mathrm{M}$ & $-0,000211$ & $-0,000210$ & $-0,000210$ & 0,000407 & $-0,000204$ & $-0,000203$ & $-0,000200$ & $-0,000205$ & $-0,000202$ & $-0,000212$ & 0,002692 & 0,001308 & $-0,000100$ \\
\hline
\end{tabular}

Raiz do Erro Quadrático Médio

MDNS MDNS- MDNS- MDNS- MDNS- MDNS- MDNS- MDNS- MDNS- MDNS- MDNS- MDNS- MDNS-

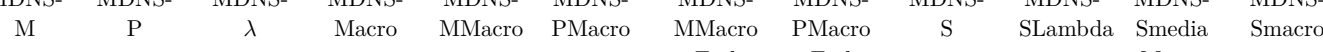
End End $\quad$ Macro

\begin{tabular}{llllllllllllll}
\hline $\mathbf{1 M}$ & 0,001994 & 0,001993 & 0,001993 & $\mathbf{0 , 0 0 1 5 3 2}$ & 0,001987 & 0,001978 & 0,001961 & 0,001943 & 0,001960 & 0,001994 & 0,002699 & 0,002539 & 0,001973
\end{tabular}

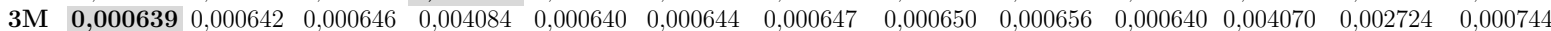

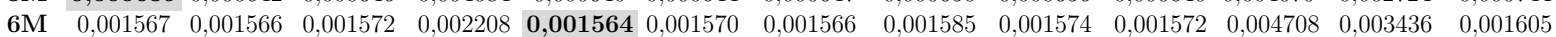
$\begin{array}{llllllllllllllll}\mathbf{9 M} & 0,001670 & 0,001665 & 0,001670 & 0,003853 & 0,001661 & 0,001663 & \mathbf{0 , 0 0 1 6 5 3} & 0,001670 & 0,001659 & 0,001672 & 0,004736 & 0,003313 & 0,001711\end{array}$ $\begin{array}{lllllllllllllll}12 \mathrm{M} & 0,001399 & 0,001393 & 0,001396 & 0,004012 & 0,001389 & 0,001387 & \mathbf{0 , 0 0 1 3 7 6} & 0,001390 & 0,001380 & 0,001399 & 0,004307 & 0,002800 & 0,001437\end{array}$ $\begin{array}{lllllllllllllll}15 \mathrm{M} & 0,001060 & 0,001055 & 0,001057 & 0,003542 & 0,001051 & 0,001049 & \mathbf{0 , 0 0 1 0 3 8} & 0,001049 & 0,001040 & 0,001060 & 0,003609 & 0,002138 & 0,001075\end{array}$ $\begin{array}{lllllllllllllll}18 \mathrm{M} & 0,000808 & 0,000805 & 0,000805 & 0,002846 & 0,000803 & 0,000802 & 0,000795 & 0,000801 & \mathbf{0 , 0 0 0 7 9 3} & 0,000809 & 0,002851 & 0,001580 & 0,000803\end{array}$ $\begin{array}{llllllllllllll}\mathbf{2 1 M} & 0,000692 & 0,000692 & 0,000691 & 0,002137 & 0,000692 & 0,000691 & 0,000692 & 0,000692 & 0,000688 & 0,000692 & 0,002158 & 0,001182 & \mathbf{0}, 000679\end{array}$

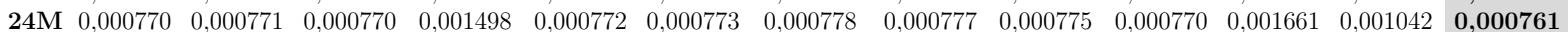
$\begin{array}{llllllllllllllll}\mathbf{2 7 M} & 0,000935 & 0,000936 & 0,000935 & 0,001128 & 0,000937 & 0,000939 & 0,000943 & 0,000943 & 0,000941 & \mathbf{0 , 0 0 0 9 3 5} & 0,001539 & 0,001193 & 0,000940\end{array}$ $\begin{array}{lllllllllllllll}\mathbf{3 0 M} & 0,001094 & 0,001094 & \mathbf{0 , 0 0 1 0 9 4} & 0,001114 & 0,001095 & 0,001097 & 0,001099 & 0,001100 & 0,001098 & 0,001094 & 0,001669 & 0,001417 & 0,001106\end{array}$ $\begin{array}{llllllllllllll}\text { 33M } & 0,001264 & 0,001262 & \mathbf{0 , 0 0 1 2 6 2} & 0,001390 & 0,001263 & 0,001264 & 0,001264 & 0,001267 & 0,001264 & 0,001263 & 0,001962 & 0,001647 & 0,001276\end{array}$ $\begin{array}{lllllllllllllll}\mathbf{3 6 M} & 0,001434 & 0,001431 & 0,001431 & 0,001795 & 0,001432 & 0,001432 & 0,001430 & 0,001434 & \mathbf{0 , 0 0 1 4 2 9} & 0,001433 & 0,002332 & 0,001901 & 0,001441\end{array}$ $\begin{array}{lllllllllllllll}39 \mathrm{M} & 0,001619 & 0,001615 & 0,001613 & 0,002272 & 0,001615 & 0,001613 & 0,001609 & 0,001613 & \mathbf{0 , 0 0 1 6 0 8} & 0,001617 & 0,002790 & 0,002216 & 0,001623\end{array}$ $\begin{array}{lllllllllllllll}48 \mathrm{M} & 0,001220 & 0,001211 & 0,001209 & 0,003038 & 0,001209 & 0,001204 & 0,001198 & 0,001202 & \mathbf{0 , 0 0 1 1 9 5} & 0,001214 & 0,003713 & 0,002611 & 0,001228\end{array}$ $\begin{array}{llllllllllllll}\mathbf{6 0 M} \mathbf{0}, 001559 & 0,001575 & 0,001576 & 0,004157 & 0,001580 & 0,001589 & 0,001611 & 0,001592 & 0,001600 & 0,001569 & 0,005351 & 0,003677 & 0,001626\end{array}$ \begin{tabular}{llllllllllllll}
$\mathbf{7 2 M} \mathbf{0 , 0 0 2 8 3 3}$ & 0,002852 & 0,002855 & 0,005033 & 0,002861 & 0,002873 & 0,002891 & 0,002880 & 0,002881 & 0,002847 & 0,006927 & 0,004676 & 0,002864 \\
\hline
\end{tabular}

Erro Absoluto Médio

$\begin{array}{ccccccccccccc}\text { MDNS } & \text { MDNS- } & \text { MDNS- } & \text { MDNS- } & \text { MDNS- } & \text { MDNS- } & \text { MDNS- } & \text { MDNS- } & \text { MDNS- } & \text { MDNS- } & \text { MDNS- } & \text { MDNS- } & \text { MDNS- } \\ & \text { M } & \mathrm{P} & \lambda & \text { Macro } & \text { MMacro } & \text { PMacro } & \text { MMacro } & \text { PMacro } & \text { S } & \text { SLambda } & \text { Smedia } & \text { Smacro } \\ & & & & & & & \text { End } & \text { End } & & & \text { Macro } & \end{array}$

\begin{tabular}{llllllllllllll}
\hline $1 \mathrm{M}$ & 0,001531 & 0,001527 & 0,001524 & $\mathbf{0 , 0 0 0 9 3 0}$ & 0,001515 & 0,001508 & 0,001501 & 0,001483 & 0,001498 & 0,001527 & 0,002123 & 0,001994 & 0,001497
\end{tabular} $\begin{array}{llllllllllllllll}3 \mathrm{M} & \mathbf{0 , 0 0 0 4 5 3} & 0,000455 & 0,000458 & 0,002865 & 0,000455 & 0,000458 & 0,000463 & 0,000459 & 0,000463 & 0,000457 & 0,003118 & 0,002175 & 0,000529\end{array}$

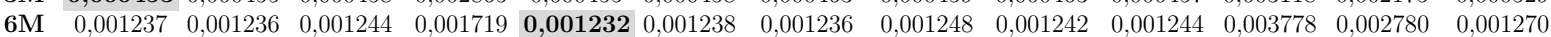
$\begin{array}{llllllllllllllll}9 \mathrm{M} & 0,001305 & 0,001300 & 0,001307 & 0,002970 & 0,001296 & 0,001296 & \mathbf{0 , 0 0 1 2 9 2} & 0,001301 & 0,001295 & 0,001306 & 0,003773 & 0,002639 & 0,001350\end{array}$ $\begin{array}{lllllllllllllll}12 \mathrm{M} & 0,001039 & 0,001036 & 0,001043 & 0,003056 & 0,001031 & 0,001033 & 0,001024 & 0,001033 & \mathbf{0 , 0 0 1 0 2 2} & 0,001043 & 0,003338 & 0,002169 & 0,001078\end{array}$ $\begin{array}{llllllllllllllll}15 \mathrm{M} & 0,000702 & 0,000700 & 0,000706 & 0,002675 & 0,000695 & 0,000697 & 0,000689 & 0,000697 & \mathbf{0 , 0 0 0 6 8 8} & 0,000707 & 0,002772 & 0,001606 & 0,000746\end{array}$ $\begin{array}{llllllllllllllll}18 \mathrm{M} & 0,000533 & 0,000531 & 0,000532 & 0,002129 & 0,000531 & 0,000531 & 0,000532 & 0,000532 & \mathbf{0 , 0 0 0 5 2 6} & 0,000533 & 0,002207 & 0,001127 & 0,000549\end{array}$ $\begin{array}{llllllllllllll}\mathbf{2 1 M} & 0,000531 & 0,000533 & 0,000531 & 0,001608 & 0,000533 & 0,000534 & 0,000535 & 0,000536 & 0,000532 & 0,000532 & 0,001652 & 0,000834 & \mathbf{0 , 0 0 0 5 2 0}\end{array}$ $\begin{array}{lllllllllllllll}\mathbf{2 4 M} & 0,000640 & 0,000641 & 0,000640 & 0,001189 & 0,000642 & 0,000643 & 0,000646 & 0,000644 & 0,000641 & 0,000641 & 0,001257 & 0,000763 & \mathbf{0 , 0 0 0 6 2 7}\end{array}$ $\begin{array}{lllllllllllllll}\mathbf{2 7 M} & 0,000720 & 0,000720 & 0,000720 & 0,000907 & 0,000719 & 0,000721 & 0,000720 & 0,000723 & 0,000720 & 0,000720 & 0,001160 & 0,000893 & \mathbf{0}, 000713\end{array}$ $\begin{array}{lllllllllllllll}\text { 30M } & 0,000748 & 0,000748 & 0,000749 & 0,000826 & 0,000745 & 0,000747 & \mathbf{0 , 0 0 0 7 4 5} & 0,000747 & 0,000746 & 0,000749 & 0,001296 & 0,001068 & 0,000749\end{array}$ $\begin{array}{lllllllllllllll}\mathbf{3 3 M} & 0,000743 & 0,000742 & 0,000745 & 0,000974 & 0,000739 & 0,000740 & \mathbf{0 , 0 0 0 7 3 7} & 0,000740 & 0,000737 & 0,000746 & 0,001480 & 0,001202 & 0,000753\end{array}$ $\begin{array}{lllllllllllllll}\mathbf{3 6 M} & 0,000756 & 0,000756 & 0,000761 & 0,001243 & 0,000753 & 0,000753 & 0,000750 & 0,000753 & \mathbf{0 , 0 0 0 7 4 9} & 0,000761 & 0,001723 & 0,001350 & 0,000777\end{array}$ $\begin{array}{llllllllllllll}39 \mathrm{M} & 0,000781 & 0,000779 & 0,000785 & 0,001562 & 0,000777 & 0,000777 & 0,000773 & 0,000776 & \mathbf{0 , 0 0 0 7 7 1} & 0,000785 & 0,002040 & 0,001508 & 0,000805\end{array}$ $\begin{array}{llllllllllllllll}48 \mathrm{M} & 0,000572 & 0,000572 & 0,000578 & 0,002200 & 0,000572 & 0,000575 & 0,000573 & 0,000575 & \mathbf{0 , 0 0 0 5 6 4} & 0,000576 & 0,002855 & 0,001679 & 0,000595\end{array}$ $\begin{array}{lllllllllllllll}60 \mathrm{M} & 0,000799 & 0,000808 & 0,000804 & 0,002934 & 0,000811 & 0,000816 & 0,000816 & 0,000818 & 0,000811 & 0,000803 & 0,003919 & 0,002106 & \mathbf{0 , 0 0 0 7 3 9}\end{array}$ $\begin{array}{llllllllllllll}\mathbf{7 2 M} & 0,001524 & 0,001529 & 0,001525 & 0,003615 & 0,001526 & 0,001530 & 0,001527 & 0,001534 & 0,001528 & 0,001524 & 0,004866 & 0,002419 & \mathbf{0 , 0 0 1 4 4 8}\end{array}$ 


\subsection{Previsão Fora da Amostra}

Além do intuito inicial de associar as oscilações da curva de juros aos eventos macroeconômicos e verificar o ajuste dos modelos na estimação, analisamos a performance da previsão fora da amostra para os treze modelos especificados anteriormente, devido a revelância que a previsão desta curva tem tanto para a execução de políticas fiscais e monetárias quanto para a análise de risco em finanças (e.g. Diebold e Li (2006)).

Estas previsões foram realizadas para horizontes de um, doze e sessenta meses em relação a cada uma das maturidades aqui analisadas. De acordo com a literatura (e.g. Diebold et al. (2006), Zhu e Rahman (2015)), se acrescentarmos variáveis macroeconômicas, aumenta-se o poder preditivo da curva de juros. Ademais, mudanças de regime já se mostram relevantes para dados mais bem comportados (e.g. Hevia et al. (2015), Zhu e Rahman (2015), Christensen (2015)), dessa forma espera-se que para curvas de juros com alterações mais frequentes em seu formato, as mudanças de regime sejam importantes para a previsão.

Para analisarmos a acurácia da previsão dos modelos são utilizados três critérios de previsão baseados no erro: ME (Mean Error), RMSE (Root Mean Squared Error), MAE (Mean Absolute Error). Apesar da raiz do erro quadrádico médio ser o principal critério de escolha, os outros dois critérios servem para corroborar a escolha do modelo.

Além disso, para evitar problemas de sobre-especificação, os modelos são reestimados com base em uma série de previsões recursivas com início em setembro de 2015, estendido de 1 a 1 mês, até agosto de 2017 (24 pontos amostrais) para todas as maturidades disponíveis e para os horizontes de previsão de um, doze e sessenta meses.

Com base nestas previsões são computadas funções perda, que corresponde a diferença entre a curva de juros observada e a prevista ao quadrado. Com esta função perda, utilizamos o procedimento do Model Confidence Set (MCS), o qual determina os melhores modelos dentre o conjunto analisado de acordo com um nível de confiança, para compararmos a acurácia preditiva de todos os modelos.

O MCS é construído com base em um conjunto de modelos, $\mathcal{M}^{0}$, e um critério para avaliar tais modelos empiricamente, aqui representado pela função perda enunciada anteriormente. O procedimento tem por base um teste de equivalência, $\delta_{\mathcal{M}}$, e uma regra de eliminação, $e_{\mathcal{M}}$. O teste de equivalência é realizado ao conjunto $\mathcal{M}=\mathcal{M}^{0}$. Caso $\delta_{\mathcal{M}}$ seja rejeitado, há evidências de que os modelos em $\mathcal{M}$ não são igualmente bons e $e_{\mathcal{M}}$ é usada para eliminar os modelos com performance ruim. Este procedimento é repetido até que $\delta_{\mathcal{M}}$ não seja mais rejeitado e os modelos "escolhidos" sejam classificados de acordo com sua acurácia preditiva.

Para o horizonte de previsão de 1 mês, o modelo MDNS-Macro se destaca para as menores maturidades e os modelos com maior flexibilidade, MDNS-MMacroEnd e 
MDNS-PMacroEnd, destacam-se para as maiores maturidades de acordo com os critérios de escolha baseados no erro. O teste do Model Confidence Set, figura 18, confirma a melhor performance do modelo MDNS-Macro para as menores maturidades e do modelo MDNS-MMacroEnd para as maturidades mais elevadas, ou seja, o Model Confidence Set indica como a melhor escolha, dentre os modelos considerados, o MDNS-Macro para maturidades menores e o MDNS-MMacroEnd para as maturidades mais elevadas.

Para a previsão de 12 meses, nas 14 primeiras maturidades o MDNS-Macro apresenta menor erro de previsão, tabela 4, para todos os critérios de escolha. Para as maturidades mais elevadas, o MDNS-S passa a apresentar menor erro de previsão, pois mesmo incorporando menos informação de dados externos, permite que o modelo seja mais flexível.

Já pelo Model Confidence Set, no horizonte de 12 meses, nota-se pela figura 19, que os modelos MDNS e MDNS-PMacro e MDNS-PMacroEnd se destacam para a maturidade até 6 meses, no entanto o modelo de maior destaque para a maioria das maturidades é o MDNS-Smacro, que incorpora além das variáveis macroeconômicas, a mudança de regime aqui proposta baseada no trabalho de So et al. (1998). Com base neste teste, nota-se também que houve uma possível sobre-especificação dos dados nos critérios de escolha baseados nos erros de previsão.

Para um horizonte de previsão de 60 meses, os modelos que se destacam, de acordo com os critérios de escolha da tabela 5, são novamente os modelos MDNS-Macro e MDNSS. No entanto, de acordo com o Model Confidence Set, figura 20, o modelo com melhor acurácia preditiva na maioria das maturidades é o MDNS- $\lambda$, indicando novamente sobreespecificação dos dados nos critérios de escolha baseados nos erros de previsão.

Dessa forma, em todos os horizontes de previsão aqui analisados, a incorporação de mudança de regime e inclusive das mudanças de regime aqui propostas (modelos MDNSMMacroEnd e MDNS-Smacro), apresentam melhor performance preditiva na maioria das maturidades pelo procedimento baseado no Model Confidence Set. Além disso, a incorporação de variáveis macroeconômicas se mostra relevante para os horizontes de 1 e 12 meses. Cabe destacar a melhor acurácia preditiva do MDNS- $\lambda$ para o horizonte de 60 meses, em contraponto ao fator de decaimento escolhido de forma ad hoc considerado no artigo de Diebold e Li (2006) para dados de curva de juros dos EUA. 
Tabela 3 - Teste de Especificação - Horizonte Previsão de 1 mês*

\begin{tabular}{|c|c|c|c|c|c|c|c|c|c|c|c|c|}
\hline \multicolumn{13}{|c|}{ Erro Médio } \\
\hline & MDNS & $\begin{array}{c}\text { MDNS- } \\
\text { M }\end{array}$ & $\begin{array}{l}\text { MDNS- } \\
\text { P }\end{array}$ & $\begin{array}{c}\text { MDNS- } \\
\lambda\end{array}$ & $\begin{array}{l}\text { MDNS- } \\
\text { Macro }\end{array}$ & $\begin{array}{l}\text { MDNS- } \\
\text { MMacro }\end{array}$ & $\begin{array}{l}\text { MDNS- } \\
\text { PMacro }\end{array}$ & $\begin{array}{l}\text { MDNS- } \\
\text { MMacro } \\
\text { End }\end{array}$ & $\begin{array}{l}\text { MDNS- } \\
\text { PMacro } \\
\text { End }\end{array}$ & $\begin{array}{c}\text { MDNS- } \\
\mathrm{S}\end{array}$ & $\begin{array}{l}\text { MDNS- } \\
\text { Smedia } \\
\text { Macro }\end{array}$ & $\begin{array}{l}\text { MDNS- } \\
\text { Smacro }\end{array}$ \\
\hline $1 \mathrm{M}$ & -0.003939 & -0.002799 & -0.002448 & -0.002820 & 0.002611 & 0.001749 & -0.001915 & 0.002165 & -0.000206 & -0.003751 & 0.002248 & -0.000437 \\
\hline $3 \mathrm{M}$ & -0.006278 & -0.005204 & -0.004893 & 0.002749 & -0.000028 & -0.000802 & -0.004158 & -0.001366 & -0.002593 & -0.006113 & -0.000407 & -0.000839 \\
\hline $6 \mathrm{M}$ & -0.007760 & -0.006778 & -0.006514 & -0.005651 & -0.001947 & -0.002616 & -0.005569 & -0.003678 & -0.004194 & -0.007622 & -0.002328 & -0.007621 \\
\hline $9 \mathrm{M}$ & -0.007349 & -0.006450 & -0.006222 & -0.009367 & -0.001946 & -0.002535 & -0.005145 & -0.003820 & -0.003933 & -0.007229 & -0.002313 & -0.009697 \\
\hline $12 \mathrm{M}$ & -0.006708 & -0.005885 & -0.005684 & -0.010598 & -0.001684 & -0.002212 & -0.004528 & -0.003535 & -0.003455 & -0.006602 & -0.002028 & -0.010036 \\
\hline $15 \mathrm{M}$ & -0.005828 & -0.005072 & -0.004893 & -0.010329 & -0.001151 & -0.001629 & -0.003693 & -0.002862 & -0.002740 & -0.005732 & -0.001467 & -0.009230 \\
\hline $18 \mathrm{~N}$ & -0.004868 & -0.004172 & -0.004011 & -0.009223 & -0.000504 & -0.000943 & -0.002789 & -0.001996 & -0.001939 & -0.004781 & -0.000794 & -0.007769 \\
\hline $21 \mathrm{M}$ & -0.003849 & -0.003207 & -0.003061 & -0.007587 & 0.000233 & -0.000173 & -0.001830 & -0.000987 & -0.001069 & -0.003768 & -0.000030 & -0.005881 \\
\hline $24 \mathrm{M}$ & -0.002871 & -0.002277 & -0.002143 & -0.005702 & 0.000958 & 0.000580 & -0.000913 & 0.000042 & -0.000228 & -0.002795 & 0.000720 & -0.003808 \\
\hline $27 \mathrm{~N}$ & & -0.002050 & -0.001926 & -0.004355 & 0.001003 & 0.000648 & -0.000700 & 0.000406 & -0.000081 & -0.002529 & 0.000785 & -0.002315 \\
\hline $30 \mathrm{I}$ & -0.002201 & -0.001688 & -0.001572 & -0.002787 & 0.001203 & 0.000868 & -0.000353 & 0.000931 & 0.000209 & -0.002132 & 0.001004 & -0.000632 \\
\hline $33 \mathrm{M}$ & -0.001757 & -0.001278 & -0.001169 & -0.001142 & 0.001468 & 0.001152 & 0.000043 & 0.001518 & 0.000555 & -0.001691 & 0.001284 & 0.001109 \\
\hline $36 \mathrm{M}$ & -0.001525 & -0.001076 & -0.000974 & 0.000289 & 0.001541 & 0.001242 & 0.000231 & 0.001905 & 0.000700 & -0.001462 & 0.001370 & 0.002619 \\
\hline $39 \mathrm{M}$ & -0.001622 & -0.001200 & -0.001104 & 0.001363 & 0.001302 & 0.001017 & 0.000096 & 0.001968 & 0.000526 & -0.001562 & 0.001142 & 0.003759 \\
\hline $48 \mathrm{~N}$ & -0.001778 & -0.001421 & -0.001341 & 0.004428 & 0.000805 & 0.000558 & -0.000150 & 0.002287 & 0.000191 & -0.001724 & 0.000664 & 0.006975 \\
\hline $60 \mathrm{M}$ & -0.003046 & -0.002750 & -0.002685 & 0.006641 & -0.000777 & -0.000987 & -0.001494 & 0.001575 & -0.001233 & -0.002999 & -0.000909 & 0.009320 \\
\hline $72 \mathrm{M}$ & -0.004013 & -0.003759 & -0.003706 & 0.008321 & -0.001959 & -0.002139 & -0.002507 & 0.001054 & -0.002299 & -0.003972 & -0.002091 & 0.011090 \\
\hline
\end{tabular}

Raiz do Erro Quadrático Médio

\begin{tabular}{|c|c|c|c|c|c|c|c|c|c|c|c|c|}
\hline & MDNS & $\begin{array}{c}\text { MDNS- } \\
\text { M }\end{array}$ & $\begin{array}{l}\text { MDNS- } \\
\text { P }\end{array}$ & $\begin{array}{c}\text { MDNS- } \\
\lambda\end{array}$ & $\begin{array}{l}\text { MDNS- } \\
\text { Macro }\end{array}$ & $\begin{array}{l}\text { MDNS- } \\
\text { MMacro }\end{array}$ & $\begin{array}{l}\text { MDNS- } \\
\text { PMacro }\end{array}$ & $\begin{array}{l}\text { MDNS- } \\
\text { MMacro } \\
\text { End }\end{array}$ & $\begin{array}{l}\text { MDNS- } \\
\text { PMacro } \\
\text { End }\end{array}$ & $\begin{array}{c}\text { MDNS- } \\
\mathrm{S}\end{array}$ & $\begin{array}{l}\text { MDNS- } \\
\text { Smedia } \\
\text { Macro }\end{array}$ & $\begin{array}{l}\text { MDNS- } \\
\text { Smacro }\end{array}$ \\
\hline $1 \mathrm{M}$ & 0.007519 & 0.005967 & & 0.004683 & 0.002618 & 0.001868 & 0.004687 & 0.002224 & 0.002607 & 0.007279 & 0.002257 & 0.002692 \\
\hline $3 \mathrm{M}$ & & 007403 & 006960 & .005127 & 0.000120 & 0.001199 & & & & & & \\
\hline $6 \mathrm{M}$ & & & 07981 & & & & & & & & & \\
\hline $9 \mathrm{M}$ & 0.009243 & 007991 & 007664 & 0.010 & 0.0 & & 24 & & & 009071 & 02399 & 9929 \\
\hline $12 \mathrm{M}$ & & 007395 & 007107 & 011162 & & & & & & & & \\
\hline $15 \mathrm{M}$ & & 006 & 06 & 09 & 0.0 & & 04677 & & 3361 & & 592 & 362 \\
\hline $18 \mathrm{M}$ & 0.006620 & .005641 & .005408 & 0.009650 & 0.000546 & & & & 0.002545 & 0.006492 & 0.000920 & 0.007865 \\
\hline $21 \mathrm{M}$ & 0.005616 & .004712 & 0.004501 & 0.007999 & 0.000243 & 0.000510 & 0.002799 & 0.001039 & 0.001736 & 0.005498 & 0.000314 & 0.005954 \\
\hline $24 \mathrm{M}$ & & & & & & & & & & & & \\
\hline $27 \mathrm{M}$ & 004230 & 003459 & .003285 & 782 & & & 0.001596 & 0.00 & 0.00 & 0.004129 & 0.000786 & 0.002346 \\
\hline $30 \mathrm{M}$ & 0.003855 & 0.003144 & 0.002987 & 0.003375 & 0.001230 & 0.000871 & 0.001345 & 0.000940 & 0.000752 & 0.003762 & 0.001004 & 0.000704 \\
\hline $33 \mathrm{M}$ & .003422 & .002770 & 002629 & 0.002106 & & & 0.001102 & & 0.000795 & 0.003336 & 0.001290 & 0.001124 \\
\hline $36 \mathrm{M}$ & & & & & & & & & & & 374 & 624 \\
\hline $39 \mathrm{M}$ & 0.003082 & 002505 & 002383 & & 0.001357 & & 0.000851 & & 0.000651 & 0.003006 & 0.001156 & 0.003760 \\
\hline $48 \mathrm{M}$ & 0.002947 & 0.002449 & 0.002346 & 0.004707 & 0.000878 & 0.000570 & 0.000666 & 0.002312 & 0.000325 & 0.002881 & 0.000684 & 0.006975 \\
\hline $60 \mathrm{M}$ & & & & & 0.000854 & & & & & & & \\
\hline $72 \mathrm{M}$ & 0.004366 & 0.004038 & 0.003972 & 0.008438 & 0.002020 & 0.002163 & 0.002512 & 0.001181 & 0.002302 & 0.004318 & 0.002116 & 0.011091 \\
\hline
\end{tabular}

Erro Absoluto Médio

MDNS MDNS- MDNS- MDNS- MDNS- MDNS- MDNS- MDNS- MDNS- MDNS- MDNS- MDNS-

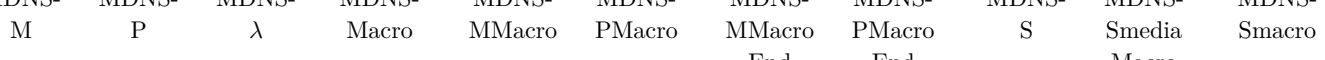

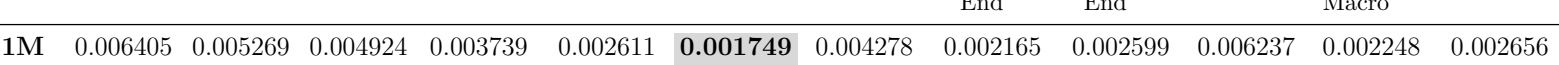
$\begin{array}{llllllllllllll}3 \mathrm{M} & 0.006332 & 0.005266 & 0.004951 & 0.004328 & \mathbf{0 . 0 0 0 1 1 6} & 0.000891 & 0.004201 & 0.001366 & 0.002675 & 0.006177 & 0.000481 & 0.002748\end{array}$ $\begin{array}{lllllllllllllll}\mathbf{6 M} & 0.007760 & 0.006778 & 0.006514 & 0.005651 & \mathbf{0 . 0 0 1 9 4 7} & 0.002616 & 0.005569 & 0.003678 & 0.004194 & 0.007622 & 0.002328 & 0.007621\end{array}$ $\begin{array}{lllllllllllll}\mathbf{9 M} & 0.007349 & 0.006450 & 0.006222 & 0.009367 & \mathbf{0 . 0 0 1 9 4 6} & 0.002535 & 0.005145 & 0.003820 & 0.003933 & 0.007229 & 0.002313 & 0.009697\end{array}$ $\begin{array}{llllllllllllll}12 \mathrm{M} & 0.006708 & 0.005885 & 0.005684 & 0.010598 & \mathbf{0 . 0 0 1 6 8 4} & 0.002212 & 0.004528 & 0.003535 & 0.003455 & 0.006602 & 0.002028 & 0.010036\end{array}$ $\begin{array}{llllllllllllll}\text { 15M } & 0.005828 & 0.005072 & 0.004893 & 0.010329 & \mathbf{0 . 0 0 1 1 5 1} & 0.001629 & 0.003693 & 0.002862 & 0.002740 & 0.005732 & 0.001467 & 0.009230\end{array}$ $\begin{array}{llllllllllllll}\text { 18M } & 0.004868 & 0.004172 & 0.004011 & 0.009223 & \mathbf{0 . 0 0 0 5 0 4} & 0.000943 & 0.002789 & 0.001996 & 0.001939 & 0.004781 & 0.000794 & 0.007769\end{array}$ $\begin{array}{llllllllllllll}21 \mathrm{M} & 0.004089 & 0.003452 & 0.003300 & 0.007587 & \mathbf{0 . 0 0 0 2 3 3} & 0.000480 & 0.002118 & 0.000987 & 0.001368 & 0.004004 & 0.000312 & 0.005881\end{array}$ $\begin{array}{llllllllllllll}\text { 24M } & 0.003790 & 0.003199 & 0.003062 & 0.005702 & 0.000958 & 0.000580 & 0.001855 & \mathbf{0 . 0 0 0 1 9 5} & 0.001172 & 0.003712 & 0.000720 & 0.003808\end{array}$ $\begin{array}{llllllllllllll}\mathbf{2 7 M} & 0.003335 & 0.002786 & 0.002662 & 0.004355 & 0.001003 & 0.000648 & 0.001435 & \mathbf{0 . 0 0 0 4 0 6} & 0.000810 & 0.003264 & 0.000785 & 0.002315\end{array}$ $\begin{array}{llllllllllllll}\text { 30M } & 0.003165 & 0.002653 & 0.002540 & 0.002787 & 0.001203 & 0.000868 & 0.001298 & 0.000931 & 0.000723 & 0.003099 & 0.001004 & \mathbf{0 . 0 0 0 6 3 2}\end{array}$ $\begin{array}{lllllllllllllll}\text { 33M } & 0.002936 & 0.002458 & 0.002354 & 0.001770 & 0.001468 & 0.001152 & 0.001102 & 0.001518 & \mathbf{0 . 0 0 0 5 6 9} & 0.002876 & 0.001284 & 0.001109\end{array}$ $\begin{array}{lllllllllllllll}36 \mathrm{M} & 0.002828 & 0.002379 & 0.002284 & 0.001754 & 0.001541 & 0.001242 & 0.001024 & 0.001905 & \mathbf{0 . 0 0 0 7 0 0} & 0.002772 & 0.001370 & 0.002619\end{array}$ $\begin{array}{lllllllllllll}39 \mathrm{M} & 0.002621 & 0.002199 & 0.002112 & 0.001634 & 0.001302 & 0.001017 & 0.000845 & 0.001968 & \mathbf{0 . 0 0 0 5 2 6} & 0.002569 & 0.001142 & 0.003759\end{array}$ $\begin{array}{llllllllllllll}\text { 48M } & 0.002350 & 0.001995 & 0.001925 & 0.004428 & 0.000805 & 0.000558 & 0.000649 & 0.002287 & \mathbf{0 . 0 0 0 2 6 3} & 0.002308 & 0.000664 & 0.006975\end{array}$ $\begin{array}{llllllllllllllll}\text { 60M } & 0.003046 & 0.002750 & 0.002685 & 0.006641 & \mathbf{0 . 0 0 0 7 7 7} & 0.000987 & 0.001494 & 0.001575 & 0.001233 & 0.002999 & 0.000909 & 0.009320\end{array}$ $\begin{array}{lllllllllllllll}72 \mathrm{M} & 0.004013 & 0.003759 & 0.003706 & 0.008321 & 0.001959 & 0.002139 & 0.002507 & \mathbf{0 . 0 0 1 0 5 4} & 0.002299 & 0.003972 & 0.002091 & 0.011090\end{array}$

*O modelo MDNS-S $\lambda$ não convergiu para as previsões. Os valores destacados apresentam erro médio, raiz do erro do erro quadrático médio ou erro absoluto médio mínimo, de acordo com a indicação da tabela, para cada maturidade. 
Tabela 4 - Teste de Especificação - Horizonte Previsão de 12 meses*

\begin{tabular}{|c|c|c|c|c|c|c|c|c|c|c|c|c|}
\hline \multicolumn{13}{|c|}{ Erro Médio } \\
\hline & MDNS & $\begin{array}{l}\text { MDNS- } \\
\text { M }\end{array}$ & $\begin{array}{l}\text { MDNS- } \\
\text { P }\end{array}$ & $\begin{array}{c}\text { MDNS- } \\
\lambda\end{array}$ & $\begin{array}{l}\text { MDNS- } \\
\text { Macro }\end{array}$ & $\begin{array}{l}\text { MDNS- } \\
\text { MMacro }\end{array}$ & $\begin{array}{l}\text { MDNS- } \\
\text { PMacro }\end{array}$ & $\begin{array}{l}\text { MDNS- } \\
\text { MMacro } \\
\text { End }\end{array}$ & $\begin{array}{l}\text { MDNS- } \\
\text { PMacro } \\
\text { End }\end{array}$ & $\begin{array}{l}\text { MDNS- } \\
\mathrm{S}\end{array}$ & $\begin{array}{l}\text { MDNS- } \\
\text { Smedia } \\
\text { Macro }\end{array}$ & $\begin{array}{l}\text { MDNS- } \\
\text { Smacro }\end{array}$ \\
\hline $1 \mathrm{M}$ & -0.013453 & -0.022373 & -0.036912 & -0.026520 & -0.000996 & -0.020082 & -0.004663 & -0.024004 & -0.002470 & -0.013076 & -0.038745 & -0.014409 \\
\hline $3 \mathrm{M}$ & -0.016449 & -0.024534 & & -0.029988 & -0.00 & -0.022240 & -0.008359 & -0.026055 & -0.006109 & -0.016015 & -0.040246 & -0.017478 \\
\hline $6 \mathrm{M}$ & -0.019861 & -0.0268 & -0.038882 & -0.028273 & -0.008589 & -0.024817 & -0.012911 & -0.028480 & -0.010601 & -0.019368 & -0.041775 & -0.021046 \\
\hline $9 \mathrm{M}$ & -0.021746 & -0.027854 & -0.038640 & -0.028314 & -0.011388 & -0.026248 & -0.015984 & -0.029769 & -0.013637 & -0.021218 & -0.042204 & -0.023215 \\
\hline $12 \mathrm{M}$ & -0.022659 & -0.0280 & -0.037745 & -0.028588 & -0.013284 & -0.026980 & -0.018089 & -0.030369 & -0.015721 & -0.022113 & -0.041998 & -0.024503 \\
\hline $15 \mathrm{M}$ & -0.022860 & -0.0275 & -0.036402 & -0.028497 & -0.014491 & -0.027195 & -0.019455 & -0.030462 & -0.017077 & -0.022309 & -0.041342 & -0.025134 \\
\hline $18 \mathrm{M}$ & & & & & & & -0.020380 & -0.030304 & -0.018002 & -0.022122 & -0.040497 & -0.025398 \\
\hline 21 & & & & & & & & & & -0.021602 & -0.039468 & -0.025331 \\
\hline 24 & S & -0.0248 & -0.0316 & -0.026491 & -0.016040 & -0.026531 & -0.021272 & -0.029478 & -0.018910 & -0.020992 & -0.038465 & -0.025163 \\
\hline $27 \mathrm{M}$ & -0.020863 & -0.023851 & -0.030104 & -0.025576 & -0.016267 & -0.026209 & -0.021544 & -0.029064 & -0.019194 & -0.020359 & -0.037530 & -0.024955 \\
\hline $30 \mathrm{M}$ & -0.020234 & -0.022931 & -0.028729 & -0.024662 & -0.016462 & -0.025925 & -0.021770 & -0.028696 & -0.019434 & -0.019749 & -0.036687 & -0.024748 \\
\hline 33 & & -0.021916 & & & & & -0.021796 & & -0.019475 & -0.019006 & -0.035766 & -0.024385 \\
\hline 36 & -0.018792 & -0.021016 & -0.026059 & -0.022620 & -0.016503 & -0.025180 & -0.021842 & & -0.019537 & -0.018347 & -0.034969 & -0.024080 \\
\hline 39 & -0.018252 & -0.0202 & -0.025011 & -0.021778 & -0.016625 & -0.024977 & -0.021970 & -0.027529 & -0.019680 & -0.017826 & -0.034344 & -0.023891 \\
\hline 48 & -0.017212 & & -0.022760 & -0.019873 & -0.017283 & -0.024861 & -0.022622 & -0.027241 & -0.020376 & -0.016843 & -0.033176 & -0.023762 \\
\hline $60 \mathrm{~N}$ & & & & -0.018151 & -0.018262 & -0.025127 & -0.023569 & -0.027330 & -0.021372 & -0.016171 & -0.032414 & -0.023960 \\
\hline $72 \mathrm{M}$ & -0.015869 & -0.016755 & -0.019534 & -0.016758 & -0.018905 & -0.025281 & -0.024178 & -0.027353 & -0.022019 & -0.015611 & -0.031833 & -0.024039 \\
\hline
\end{tabular}

Raiz do Erro Quadrático Médio

MDNS MDNS- MDNS- MDNS- MDNS- MDNS- MDNS- MDNS- MDNS- MDNS- MDNS- MDNS-

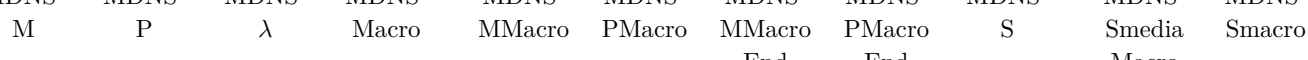

\begin{tabular}{lllllllllllll}
\hline $\mathbf{1 M}$ & 0.019581 & 0.026511 & 0.044188 & 0.032294 & $\mathbf{0 . 0 0 6 5 3 3}$ & 0.025864 & 0.010366 & 0.030501 & 0.009044 & 0.019233 & 0.047274 & 0.020489
\end{tabular} $\begin{array}{llllllllllllll}3 \mathrm{M} & 0.023064 & 0.029457 & 0.045828 & 0.035435 & \mathbf{0 . 0 0 9 2 8 0} & 0.028638 & 0.014229 & 0.033147 & 0.012405 & 0.022658 & 0.049301 & 0.024097\end{array}$ $\begin{array}{llllllllllllll}\mathbf{6 M} & 0.026448 & 0.032168 & 0.046784 & 0.034985 & \mathbf{0 . 0 1 2 9 6 5} & 0.031297 & 0.018585 & 0.035626 & 0.016405 & 0.025979 & 0.050765 & 0.027576\end{array}$ $\begin{array}{llllllllllllll}\mathbf{9 M} & 0.027769 & 0.032904 & 0.046004 & 0.034793 & \mathbf{0 . 0 1 5 1 3 0} & 0.032216 & 0.021081 & 0.036376 & 0.018719 & 0.027260 & 0.050565 & 0.029156\end{array}$ $\begin{array}{lllllllllllllll}12 \mathrm{M} & 0.028114 & 0.032697 & 0.044496 & 0.034426 & \mathbf{0 . 0 1 6 5 2 4} & 0.032397 & 0.022644 & 0.036399 & 0.020180 & 0.027585 & 0.049695 & 0.029878\end{array}$ $\begin{array}{llllllllllllll}15 \mathrm{M} & 0.027858 & 0.031937 & 0.042618 & 0.033729 & \mathbf{0 . 0 1 7 3 9 4} & 0.032171 & 0.023626 & 0.036022 & 0.021107 & 0.027321 & 0.048478 & 0.030062\end{array}$ $\begin{array}{llllllllllllll}18 \mathrm{M} & 0.027320 & 0.030957 & 0.040674 & 0.032849 & \mathbf{0 . 0 1 7 9 9 1} & 0.031806 & 0.024300 & 0.035515 & 0.021751 & 0.026785 & 0.047191 & 0.029996\end{array}$ $\begin{array}{llllllllllllll}\mathbf{2 1 M} & 0.026431 & 0.029686 & 0.038565 & 0.031647 & \mathbf{0 . 0 1 8 2 2 0} & 0.031186 & 0.024573 & 0.034763 & 0.022013 & 0.025905 & 0.045724 & 0.029597\end{array}$ $\begin{array}{llllllllllllll}\text { 24M } & 0.025434 & 0.028362 & 0.036511 & 0.030348 & \mathbf{0 . 0 1 8 2 9 7} & 0.030524 & 0.024666 & 0.033979 & 0.022102 & 0.024922 & 0.044288 & 0.029093\end{array}$ $\begin{array}{llllllllllllll}\mathbf{2 7 M} & 0.024610 & 0.027235 & 0.034750 & 0.029205 & \mathbf{0 . 0 1 8 4 7 3} & 0.030055 & 0.024849 & 0.033397 & 0.022293 & 0.024116 & 0.043111 & 0.028736\end{array}$ $\begin{array}{llllllllllllll}\text { 30M } & 0.023837 & 0.026202 & 0.033165 & 0.028116 & \mathbf{0 . 0 1 8 6 3 2} & 0.029660 & 0.025009 & 0.032900 & 0.022460 & 0.023362 & 0.042073 & 0.028409\end{array}$ $\begin{array}{llllllllllllll}33 \mathrm{M} & 0.022960 & 0.025099 & 0.031570 & 0.026925 & \mathbf{0 . 0 1 8 6 3 2} & 0.029164 & 0.025003 & 0.032307 & 0.022469 & 0.022504 & 0.040987 & 0.027953\end{array}$ $\begin{array}{llllllllllllll}\mathbf{3 6 M} & 0.022183 & 0.024122 & 0.030161 & 0.025839 & \mathbf{0 . 0 1 8 6 6 3} & 0.028761 & 0.025018 & 0.031817 & 0.022498 & 0.021748 & 0.040052 & 0.027573\end{array}$ $\begin{array}{llllllllllllll}39 \mathrm{M} & 0.021601 & 0.023362 & 0.029019 & 0.024955 & \mathbf{0 . 0 1 8 8 2 1} & 0.028539 & 0.025160 & 0.031515 & 0.022656 & 0.021187 & 0.039347 & 0.027359\end{array}$ $\begin{array}{lllllllllllllll}48 \mathrm{M} & 0.020252 & 0.021616 & 0.026346 & 0.022804 & \mathbf{0 . 0 1 9 4 1 1} & 0.028208 & 0.025680 & 0.030976 & 0.023218 & 0.019891 & 0.037805 & 0.026992\end{array}$ $\begin{array}{lllllllllllllll}\text { 60M } & 0.019401 & 0.020395 & 0.024273 & 0.021064 & 0.020469 & 0.028440 & 0.026659 & 0.031004 & 0.024244 & \mathbf{0 . 0 1 9 1 0 2} & 0.036869 & 0.027133\end{array}$ $\begin{array}{lllllllllllll}\mathbf{7 2 M} & 0.018759 & 0.019504 & 0.022791 & 0.019744 & 0.021220 & 0.028624 & 0.027340 & 0.031037 & 0.024963 & \mathbf{0 . 0 1 8 5 0 8} & 0.036218 & 0.027225\end{array}$

Erro Absoluto Médio

\begin{tabular}{|c|c|c|c|c|c|c|c|c|c|c|c|c|}
\hline & MDNS & $\begin{array}{c}\text { MDNS- } \\
\text { M }\end{array}$ & $\begin{array}{c}\text { MDNS- } \\
\text { P }\end{array}$ & $\begin{array}{c}\text { MDNS- } \\
\lambda\end{array}$ & $\begin{array}{l}\text { MDNS- } \\
\text { Macro }\end{array}$ & $\begin{array}{l}\text { MDNS- } \\
\text { MMacro }\end{array}$ & $\begin{array}{l}\text { MDNS- } \\
\text { PMacro }\end{array}$ & $\begin{array}{l}\text { MDNS- } \\
\text { MMacro } \\
\text { End }\end{array}$ & $\begin{array}{l}\text { MDNS- } \\
\text { PMacro } \\
\text { End }\end{array}$ & $\begin{array}{c}\text { MDNS- } \\
\mathrm{S}\end{array}$ & $\begin{array}{l}\text { MDNS- } \\
\text { Smedia } \\
\text { Macro }\end{array}$ & $\begin{array}{l}\text { MDNS- } \\
\text { Smacro }\end{array}$ \\
\hline & & & & & & & & 0.009031 & 0.007775 & & 0.038745 & \\
\hline & & & & & & & & & & & & \\
\hline & & & & & & & & & & & & \\
\hline & & & & & & & & & & & & \\
\hline & & & & & & & & & & & & \\
\hline & & 6 & 02 & 22 & & & & & & & & \\
\hline & & & & & & & & & & & & \\
\hline & & & & & & & & & & & & \\
\hline & & & & & & & & & & & & \\
\hline & & & & & & & & & & & & \\
\hline & & & & & & & & & & & & \\
\hline & & 021916 & 0.027313 & 023603 & & & & & & & & 0.024385 \\
\hline & & & & & & & & & & & & \\
\hline & & & & & & & & & & & & \\
\hline & & & & & & & & & & & & \\
\hline & & & & & & & & & & & & \\
\hline $72 \mathrm{M}$ & 0.015969 & 0.016869 & 0.019646 & 0.016974 & 0.018982 & 0.025358 & 0.024262 & 0.024262 & 0.022106 & 0.015704 & 0.031911 & 0.024107 \\
\hline
\end{tabular}

$\overline{\text { *O modelo MDNS-S } \lambda \text { não convergiu para as previsões. Os valores destacados apresentam erro médio, raiz do erro do erro quadrático }}$ médio ou erro absoluto médio mínimo, de acordo com a indicação da tabela, para cada maturidade. 
Tabela 5 - Teste de Especificação - Horizonte Previsão de 60 meses*

\begin{tabular}{|c|c|c|c|c|c|c|c|c|c|c|c|c|}
\hline \multicolumn{13}{|c|}{ Erro Médio } \\
\hline & MDNS & $\begin{array}{c}\text { MDNS- } \\
\text { M }\end{array}$ & $\begin{array}{l}\text { MDNS- } \\
\text { P }\end{array}$ & $\begin{array}{c}\text { MDNS- } \\
\lambda\end{array}$ & $\begin{array}{l}\text { MDNS- } \\
\text { Macro }\end{array}$ & $\begin{array}{l}\text { MDNS- } \\
\text { MMacro }\end{array}$ & $\begin{array}{l}\text { MDNS- } \\
\text { PMacro }\end{array}$ & $\begin{array}{l}\text { MDNS- } \\
\text { MMacro } \\
\text { End }\end{array}$ & $\begin{array}{l}\text { MDNS- } \\
\text { PMacro } \\
\text { End }\end{array}$ & $\begin{array}{l}\text { MDNS- } \\
\mathrm{S}\end{array}$ & $\begin{array}{l}\text { MDNS- } \\
\text { Smedia } \\
\text { Macro }\end{array}$ & $\begin{array}{l}\text { MDNS- } \\
\text { Smacro }\end{array}$ \\
\hline $1 \mathrm{M}$ & -0.010328 & -0.011717 & -0.049026 & -0.018766 & 0.000285 & 0.710887 & 0.018920 & 0.001352 & 0.033635 & 0.014653 & -0.065121 & 0.033941 \\
\hline $3 \mathrm{M}$ & -0.010438 & -0.011722 & -0.046188 & -0.018093 & -0.000277 & 0.677881 & 0.018561 & 0.000695 & 0.041312 & 0.014246 & -0.058911 & 0.031030 \\
\hline $6 \mathrm{M}$ & -0.010882 & -0.012028 & -0.042748 & -0.017822 & -0.001357 & 0.643650 & 0.017734 & -0.000474 & 0.042538 & 0.013144 & -0.051463 & 0.026942 \\
\hline $9 \mathrm{M}$ & -0.011430 & -0.012457 & -0.039964 & -0.017653 & -0.002490 & 0.615467 & 0.016805 & -0.001646 & 0.041871 & 0.011763 & -0.045674 & 0.023317 \\
\hline $12 \mathrm{M}$ & -0.011792 & -0.012713 & -0.037457 & -0.017352 & -0.003386 & 0.591749 & 0.016075 & -0.002547 & 0.041159 & 0.010467 & -0.040932 & 0.020346 \\
\hline $15 \mathrm{M}$ & -0.011964 & -0.012794 & -0.035151 & -0.016937 & -0.004045 & 0.571641 & 0.015551 & -0.003186 & 0.040450 & 0.009307 & -0.036983 & 0.017953 \\
\hline $18 \mathrm{M}$ & -0.011975 & & & -0.016433 & -0.004497 & 0.554515 & 0.015211 & -0.003601 & 0.039708 & 0.008295 & -0.033654 & 0.016044 \\
\hline 21 & & & & 15866 & -0.004778 & 0.539869 & 0.015023 & -0.0038 & 0.038934 & 0.007422 & -0.030819 & 0.014534 \\
\hline $24 \mathrm{I}$ & -0.011720 & -0.012331 & -0.029251 & -0.015339 & -0.005005 & 0.527211 & 0.014874 & -0.004005 & 0.038063 & 0.006594 & -0.028467 & 0.013265 \\
\hline $27 \mathrm{M}$ & -0.011607 & -0.012160 & -0.027705 & -0.014885 & -0.005219 & 0.516190 & 0.014725 & -0.004161 & 0.037102 & 0.005781 & -0.026538 & 0.012162 \\
\hline $30 \mathrm{M}$ & -0.011555 & -0.012055 & -0.026391 & -0.014534 & -0.005463 & 0.506514 & 0.014538 & -0.004344 & 0.036052 & 0.004955 & -0.024989 & 0.011157 \\
\hline $33 \mathrm{~N}$ & -0.011436 & & & -0.014154 & -0.005612 & 0.498094 & 0.014437 & -0.004433 & 0.035067 & 0.004244 & -0.023632 & 0.010352 \\
\hline $36 \mathrm{I}$ & -0.011332 & -0.011741 & -0.024063 & -0.013819 & -0.005749 & 0.490675 & 0.014341 & -0.004512 & 0.034090 & 0.003570 & -0.022497 & 0.009646 \\
\hline 39 & 11235 & -0.0116 & -0.023086 & -0.013519 & -0.005872 & 0.484114 & 0.014254 & -0.004579 & 0.033140 & 0.002938 & -0.021538 & 0.009028 \\
\hline $48 \mathrm{I}$ & -0.011299 & -0.011571 & -0.021024 & -0.013100 & -0.006480 & 0.468134 & 0.013732 & -0.005032 & 0.030226 & 0.000980 & -0.019737 & 0.007270 \\
\hline $60 \mathrm{M}$ & & & & -0.012899 & -0.007245 & 0.453190 & 0.013045 & -0.005632 & 0.026917 & -0.001210 & -0.018504 & 0.005481 \\
\hline $72 \mathrm{M}$ & -0.011478 & -0.011579 & -0.017820 & -0.012540 & -0.007560 & 0.443108 & 0.012785 & -0.005819 & 0.024640 & -0.002602 & -0.017560 & 0.004481 \\
\hline
\end{tabular}

Raiz do Erro Quadrático Médio

MDNS MDNS- MDNS- MDNS- MDNS- MDNS- MDNS- MDNS- MDNS- MDNS- MDNS- MDNS$\begin{array}{llllllll}\text { M } & \mathrm{P} & \lambda & \text { Macro } & \text { MMacro PMacro MMacro PMacro } & \text { S } & \text { Smedia Smacro }\end{array}$ $\begin{array}{lll}\text { End } & \text { End } & \text { Macro }\end{array}$

\begin{tabular}{lllllllllllll}
\hline $\mathbf{1 M}$ & 0.022655 & 0.021844 & 0.053947 & 0.027105 & $\mathbf{0 . 0 1 1 7 3 0}$ & 1.033219 & 0.021309 & 0.024612 & 0.040712 & 0.035186 & 0.117316 & 0.042333
\end{tabular} $\begin{array}{llllllllllllll}\mathbf{3 M} & 0.022632 & 0.022020 & 0.051722 & 0.026745 & \mathbf{0 . 0 1 2 8 4 5} & 0.986937 & 0.021932 & 0.025090 & 0.050136 & 0.033991 & 0.108281 & 0.040162\end{array}$ $\begin{array}{llllllllllllll}\mathbf{6 M} & 0.022901 & 0.022527 & 0.048997 & 0.026890 & \mathbf{0 . 0 1 4 4 6 0} & 0.936548 & 0.022512 & 0.025689 & 0.051409 & 0.032253 & 0.097188 & 0.037123\end{array}$ $\begin{array}{llllllllllllll}\mathbf{9 M} & 0.023045 & 0.022829 & 0.046569 & 0.026778 & \mathbf{0 . 0 1 5 6 1 8} & 0.895186 & 0.022649 & 0.025889 & 0.050612 & 0.030478 & 0.088134 & 0.034267\end{array}$ $\begin{array}{lllllllllllllll}12 \mathrm{M} & 0.022985 & 0.022868 & 0.044248 & 0.026416 & \mathbf{0 . 0 1 6 4 5 8} & 0.860354 & 0.022657 & 0.025889 & 0.049756 & 0.028798 & 0.080635 & 0.031855\end{array}$ $\begin{array}{llllllllllllll}15 \mathrm{M} & 0.022709 & 0.022666 & 0.042023 & 0.025842 & \mathbf{0 . 0 1 7 0 8 8} & 0.830774 & 0.022594 & 0.025767 & 0.048849 & 0.027168 & 0.074333 & 0.029822\end{array}$ $\begin{array}{lllllllllllllll}18 \mathrm{M} & 0.022358 & 0.022374 & 0.039936 & 0.025201 & \mathbf{0 . 0 1 7 6 4 3} & 0.805503 & 0.022567 & 0.025652 & 0.047862 & 0.025702 & 0.069061 & 0.028196\end{array}$ $\begin{array}{llllllllllllll}\mathbf{2 1 M} & 0.021930 & 0.021985 & 0.037929 & 0.024502 & \mathbf{0 . 0 1 8 0 2 7} & 0.783871 & 0.022583 & 0.025457 & 0.046844 & 0.024409 & 0.064613 & 0.026911\end{array}$ $\begin{array}{llllllllllllll}\mathbf{2 4 M} & 0.021519 & 0.021601 & 0.036110 & 0.023848 & \mathbf{0 . 0 1 8 3 5 9} & 0.765192 & 0.022612 & 0.025266 & 0.045743 & 0.023262 & 0.060900 & 0.025881\end{array}$

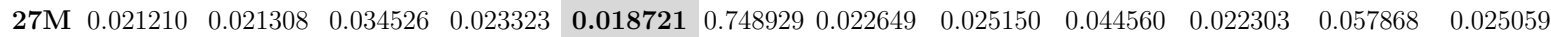
$\begin{array}{llllllllllllll}\text { 30M } & 0.020958 & 0.021066 & 0.033137 & 0.022886 & \mathbf{0 . 0 1 9 0 2 8} & 0.734724 & 0.022635 & 0.025028 & 0.043319 & 0.021473 & 0.055330 & 0.024360\end{array}$ $\begin{array}{lllllllllllllll}33 \mathrm{M} & 0.020671 & 0.020774 & 0.031787 & 0.022432 & \mathbf{0 . 0 1 9 2 0 2} & 0.722375 & 0.022641 & 0.024832 & 0.042172 & 0.020793 & 0.053127 & 0.023792\end{array}$ $\begin{array}{llllllllllllll}\mathbf{3 6 M} & 0.020410 & 0.020507 & 0.030588 & 0.022023 & \mathbf{0 . 0 1 9 3 7 0} & 0.711488 & 0.022639 & 0.024668 & 0.041030 & 0.020186 & 0.051289 & 0.023323\end{array}$ $\begin{array}{llllllllllllll}3 \text { 39M } & 0.020198 & 0.020290 & 0.029554 & 0.021681 & \mathbf{0 . 0 1 9 6 0 4} & 0.701802 & 0.022663 & 0.024608 & 0.039900 & 0.019665 & 0.049805 & 0.022956\end{array}$ $\begin{array}{llllllllllllll}\text { 48M } & 0.019750 & 0.019834 & 0.027230 & 0.020951 & 0.020244 & 0.678459 & 0.022451 & 0.024493 & 0.036549 & \mathbf{0 . 0 1 8 3 5 4} & 0.046633 & 0.021986\end{array}$ $\begin{array}{lllllllllllllll}\text { 60M } & 0.019451 & 0.019526 & 0.025247 & 0.020392 & 0.021053 & 0.656680 & 0.022186 & 0.024555 & 0.032830 & \mathbf{0 . 0 1 7 3 3 3} & 0.044210 & 0.021284\end{array}$ $\begin{array}{lllllllllllll}72 \mathrm{M} & 0.019212 & 0.019272 & 0.023784 & 0.019959 & 0.021657 & 0.641797 & 0.022254 & 0.024654 & 0.030327 & \mathbf{0 . 0 1 6 9 2 3} & 0.042700 & 0.021104\end{array}$

Erro Absoluto Médio

\begin{tabular}{|c|c|c|c|c|c|c|c|c|c|c|c|c|}
\hline & MDNS & $\begin{array}{c}\text { MDNS- } \\
\text { M }\end{array}$ & $\begin{array}{l}\text { MDNS- } \\
\text { P }\end{array}$ & $\begin{array}{l}\text { KDNS- } \\
\lambda\end{array}$ & $\begin{array}{l}\text { MDNS- } \\
\text { Macro }\end{array}$ & $\begin{array}{l}\text { MDNS- } \\
\text { MMacro }\end{array}$ & $\begin{array}{l}\text { MDNS- } \\
\text { PMacro }\end{array}$ & $\begin{array}{l}\text { MDNS- } \\
\text { MMacro } \\
\text { End }\end{array}$ & $\begin{array}{l}\text { MDNS- } \\
\text { PMacro } \\
\text { End }\end{array}$ & $\begin{array}{c}\text { MDNS- } \\
\text { S }\end{array}$ & $\begin{array}{l}\text { MDNS- } \\
\text { Smedia } \\
\text { Macro }\end{array}$ & $\begin{array}{l}\text { MDNS- } \\
\text { Smacro }\end{array}$ \\
\hline $1 \mathrm{M}$ & & & & & & & & 0.020694 & 0.033635 & & 0.079253 & \\
\hline & & & & & & & & & & & & \\
\hline & & & & & & & & & & & & \\
\hline & & & & & & & & & & & & \\
\hline $2 \mathrm{M}$ & & & & & & & & & & & & \\
\hline & & & & 60 & & & & & & & & \\
\hline & & & & & & & & & & & & \\
\hline & & & & & & & & & & & & \\
\hline & & & & & & & & & & & & \\
\hline $27 \mathrm{M}$ & & 017650 & .028405 & 019769 & & & & & & & & \\
\hline & & & & & & & & & & & & \\
\hline & & & & & & & & & & & & \\
\hline & & & & & & & & & & & & \\
\hline & & & & & & & & & & & & \\
\hline $48 \mathrm{M}$ & & 01713 & 0232 & 018313 & & & 0.0171 & & 0.030724 & 0.013196 & 460 & 016408 \\
\hline $60 \mathrm{M}$ & & & 0278 & & & & & & & & & \\
\hline $72 \mathrm{M}$ & 0.016926 & 0.016987 & 0.020801 & 0.017682 & 0.015976 & 0.443198 & 0.017053 & 0.018126 & 0.025094 & 0.012593 & 0.029296 & 0.015752 \\
\hline
\end{tabular}

$\overline{\text { *O modelo MDNS-S } \lambda \text { não convergiu para as previsões. Os valores destacados apresentam erro médio, raiz do erro do erro quadrático }}$ médio ou erro absoluto médio mínimo, de acordo com a indicação da tabela, para cada maturidade. 


\section{Conclusão}

Neste trabalho foram propostas diversas inovações para a estimação da curva de DI, baseadas em diferentes abordagens do modelo dinâmico de Nelson-Siegel. Estas especificações propostas tornam os modelos mais flexíveis, o que permite superar limitações do modelo mais simples (e.g. Diebold e Li (2006)), sendo assim mais adequadas para curvas menos estáveis que a dos EUA, onde foi inicialmente proposta.

Trabalhos com uma análise mais detalhada de extensões do modelo dinâmico de Nelson-Siegel foram mais amplamente realizadas para a estimação e previsão da curva dos EUA e de países desenvolvidos.

Pela dificuldade na inferência estatística dos modelos por sua não-linearidade, já que em todos os modelos o parâmetro de decaimento não é escolhido de forma ad hoc, e por conter dois fatores não observados, os regimes latentes e os fatores latentes da curva de juros, utilizamos a abordagem de MCMC para as estimações.

Os modelos que apresentam melhor performance dependem da maturidade analisada e, para a previsão, dependem também do horizonte de previsão considerado. A incorporação de mais flexibilidade no fator de decaimento, por meio da mudança de regime, somente se mostra relevante na estimação para maturidade de um mês. Para as outras maturidades, modelos com a incorporação de variáveis macroeconômicas e ao mesmo tempo com mudanças de regime (MDNS-PMacro e MDNS-PMacroEnd e MDNS-SMacro) apresentam melhor ajuste. Nota-se que a incorporação de maior flexibilidade, tais como nos modelos MDNS-PMacroEnd e MDNS-Smacro, mostra-se relevante para o ajuste na estimação. Dessa forma, extensões propostas neste trabalho apresentam melhor ajuste para a estimação com dados brasileiros de DI.

Para um horizonte de previsão de 1 mês, os modelos com a incorporação de fatores macroeconômicos apresentam melhor poder preditivo. O modelo MDNS-Macro apresenta melhor acurácia para maturidades menores e o MDNS-MMacroEnd, aqui proposto, que incorpora mudança de regime tanto nas variáveis macroeconômicas quanto nos fatores latentes, tem melhor poder preditivo para as maturidades mais elevadas.

Para o horizonte de 12 meses, de acordo com o Model Confidence Set, modelos com a incorporação de variáveis macroeconômicas e mudanças de regime apresentam melhor acurácia preditiva, sendo que o MDNS-Smacro se destaca para a maioria das maturidades.

Por fim, para horizonte de 60 meses, o modelo somente com a incorporação de mudança de regime no fator de decaimento $(\operatorname{MDNS}-\lambda)$ apresenta melhor acurácia preditiva para a maioria das maturidades de acordo com o Model Confidence Set. Dessa forma, nota-se uma distinção no comportamento da curva DI e da curva de juros dos EUA, em que modelos com fator de decaimento escolhidos de forma ad hoc apresentam uma boa 
performance (e.g. Diebold e Li (2006)).

Dessa forma, os modelos que incorporam maior flexibilidade se mostram relevantes para o ajuste na estimação e também para previsões em todos os horizontes de previsão aqui analisados, com destaque para MDNS-MMacroEnd, MDNS-Smacro e MDNS- $\lambda$.

Isso posto, nota-se a importância da incorporação de mudanças de regime e/ou de variáveis macroeconômicas para a estimação e previsão da taxa de juros a termo brasileira. 


\section{Referências}

ANG, A.; PIAZZENI, M. A no-arbitrage vector autoregression of term structure dynamics with macroeconomic and latent variable. Journal of Monetary Economics, p. 745-787, 2003.

ARRUDA, E. F.; FERREIRA, R. T.; CASTELAR, I. Modelos lineares e não lineares da curva de Phillips para previsão da taxa de inflação no Brasil. RBE, p. 237-252, 2011.

Bank for International Settlements. Zero-coupon yield curves estimated by central banks. 2015. < http://www.bis.org/publ/bppdf/bispap25a.pdf>. Online; accessado 25 Setembro 2017.

CALDEIRA, J. et al. Forecasting the yield curve with the arbitrage-free dynamic Nelson-Siegel model: Brazilian evidence. EconomiA, n. 17(2), p. 221-237, 2016.

CHRISTENSEN, J. H. A regime-switching model of the yield curve at the zero bound. Working Paper Federal Reserve Bank of San Francisco Series 2013-34, 2015.

CHRISTENSEN, J. H.; DIEBOLD, F. X.; RUDEBUSCH, G. D. The affine arbitrage-free class of Nelson-Siegel term structure models. Journal of Econometrics, p. 4-20, 2011.

DIEBOLD, F. X.; LI, C. Forecasting the term structure of the government bond yields. Journal of Econometrics, p. 337-369, 2006.

DIEBOLD, F. X.; LI, C.; YUE, V. Z. Global yield curve dynamics and interactions: A dynamic Nelson-Siegel approach. Journal of Econometrics, p. 351-363, 2008.

DIEBOLD, F. X.; PIAZZENI, M.; RUDEBUSCH, G. D. Modeling bond yields in finance and macroeconomics. Working Paper Federal Reserve Bank of San Francisco Series 05-008, 2005.

DIEBOLD, F. X.; RUDEBUSCH, G. D. Yield curve modeling and forecasting: the dynamic Nelson-Siegel approach. [S.l.]: Princeton University Press, 2013. (Econometric and Tinbergen Institutes lectures).

DIEBOLD, F. X.; RUDEBUSCH, G. D.; ARUOBA, S. B. The macroeconomy and yield curve: a dynamic latent factor approach. Journal of Econometrics, p. 309-338, 2006.

DUFFEE, G. R. Term premia and interest rate forecasts in affine models. The Journal of Finance, p. 405-443, 2002.

DUFFIE, D.; KAN, R. A yield-factor model of interest rates. Mathematical Finance, p. 379-406, 1996.

FARIA, A.; ALMEIDA, C. Forecasting the Brazilian term structure using macroeconomic factors. Brazilian Review of Econometric, v. 34, n. 1, 2014.

FILHO, F. de H. B. A crise econômica de 2014/2017. Estudos Avançados, v. 31, 2017. 
GONÇALVES, C.; PORTUGAL, M. S.; ARAGÓN, E. Assessing Brazilian macroeconomic dynamics using a Markov-switching DSGE model. EconomiA, p. 23-42, 2016.

HEVIA, C.; GONZALES-ROZADA, M.; SPAGNOLO, F. Estimating and forecasting the yield curve using a Markov switching dynamic Nelson and Siegel model. Journal of Applied Econometrics, p. 987-1009, 2015.

KOBAYASHI, T. Regime-switching dynamic Nelson-Siegel modeling to corporate bond yield spreads with time-varying transition probabilities. Journal of Applied Business and Economics, n. 19(5), p. 10-28, 2017.

LAURINI, M. P.; HOTTA, L. K. Modelos de fatores latentes generalizados para curvas de juros em múltplicos mercados. Anbima, 2009.

LAURINI, M. P.; HOTTA, L. K. Bayesian extensions to Diebold-Li term structure model. International Review of Financial Analysis, p. 342-350, 2010.

LEVANT, J.; MA, J. A dynamic Nelson-Siegel yield curve model with Markov switching. Economic Modelling, p. 73-87, 2017.

MATSUMURA, M. S.; MOREIRA, A. R. B. Macro factors and the Brazilian yield curve with no arbitrage models. IPEA - Discussion Paper, n. 171, 2006.

MUNCLINGER, R. Exploration of the Brazilian term structure in a hidden Markov framework. IMF Working Paper, n. 11/22, 2011.

RUDEBUSCH, G. D.; WU, T. A macro-finance model of the term structure, monetary policy and the economy. The Economic Journal, p. 906-926, 2008.

SO, M. K. P.; LAM, K.; LI, W. K. A stochastic volatility model with Markov switching. Journal of Business and Economic Statistics, p. 244-253, 1998.

VICENTE, J.; TABAK, B. M. Forecasting bond yields in the Brazilian fixed income market. International Journal of Forecasting, p. 490-497, 2008.

WICHMANN, R. M.; PORTUGAL, M. S. Política fiscal assimétrica: O caso do Brasil. RBE, p. 355-378, 2013.

ZHU, X.; RAHMAN, S. A regime-switching Nelson-Siegel term structure model of the macroeconomy. Journal of Macroeconomics, p. 1-17, 2015. 


\section{Anexo}

Figura 18 - Model Confidence Set - Horizonte de Previsão 1 mês*

\begin{tabular}{lccccccc}
\hline \multicolumn{7}{c}{ Maturidade de 1 mês } \\
\hline Modelos & Rank_M & V_M & MCS_M & Rank_R & V_R & MCS_R & Loss \\
\hline MDNS & 5 & -0.02753 & 1.0000 & 7 & 2.977764 & 0.0244 & $2.45 \mathrm{E}-05$ \\
MDNS_M & 6 & 0.323828 & 0.9998 & 11 & 5.510465 & 0.0000 & $2.55 \mathrm{E}-05$ \\
MDNS_P & 11 & 2.01872 & 0.2000 & 10 & 4.640384 & 0.0000 & $3.85 \mathrm{E}-05$ \\
MDNS_Lambda & 8 & 0.451478 & 0.9982 & 8 & 2.986229 & 0.0228 & $2.73 \mathrm{E}-05$ \\
MDNS_Macro & 1 & -8.05716 & 1.0000 & 1 & -1.21663 & 1.0000 & $6.69 \mathrm{E}-06$ \\
MDNS_MMacro & 4 & -0.6744 & 1.0000 & 5 & 2.638883 & 0.1010 & $2.11 \mathrm{E}-05$ \\
MDNS_MMacroEnd & 3 & -1.94083 & 1.0000 & 3 & 2.264121 & 0.2956 & $1.57 \mathrm{E}-05$ \\
MDNS_PMacroEnd & 9 & 1.354759 & 0.6992 & 6 & 2.750061 & 0.0692 & $4.09 \mathrm{E}-05$ \\
MDNS_S & 10 & 1.845387 & 0.2988 & 9 & 4.514676 & 0.0000 & $3.23 \mathrm{E}-05$ \\
MDNS_SmediaMacro & 7 & 0.451065 & 0.9982 & 4 & 2.530331 & 0.2956 & $2.83 \mathrm{E}-05$ \\
MDNS_Smacro & 2 & -3.69946 & 1.0000 & 2 & 1.21663 & 1.0000 & $1.08 \mathrm{E}-05$ \\
& & & & & & &
\end{tabular}

\begin{tabular}{|c|c|c|c|c|c|c|c|}
\hline \multicolumn{8}{|c|}{ Maturidade de 3 meses } \\
\hline Modelos & Rank_M & $V_{n} M$ & MCS_M & Rank_R & $V_{-} R$ & MCS_R $R$ & Loss \\
\hline MDNS & 9 & 0.310987 & 0.9990 & 5 & 2.218548 & 0.3016 & 4.29E- -05 \\
\hline MDNS_M & 5 & -0.08485 & 1.0000 & 8 & 2.701816 & 0.0666 & $3.89 \mathrm{E}-05$ \\
\hline MDNS_P & 10 & 0.546868 & 0.9812 & 2 & 1.883955 & 0.5790 & $4.85 \mathrm{E}-05$ \\
\hline MDNS_Lambda & 8 & 0.293921 & 0.9998 & 4 & 2.069529 & 0.4116 & $4.28 \mathrm{E}-05$ \\
\hline MDNS_Macro & 1 & -6.34909 & 1.0000 & 1 & -1.88395 & 1.0000 & $1.01 \mathrm{E}-05$ \\
\hline MDNS_MMacro & 3 & -1.14476 & 1.0000 & 10 & 4.171711 & 0.0000 & $3.02 \mathrm{E}-05$ \\
\hline MDNS_PMacro & 12 & 2.065681 & 0.1580 & 11 & 4.670522 & 0.0000 & $7.23 \mathrm{E}-05$ \\
\hline MDNS_MMacroEnd & 6 & 0.039199 & 1.0000 & 6 & 2.26142 & 0.2750 & 4.01E-05 \\
\hline MDNS_PMacroEnd & 11 & 0.757245 & 0.9206 & 9 & 3.465668 & 0.0016 & $4.75 \mathrm{E}-05$ \\
\hline MDNS_S & 7 & 0.210641 & 1.0000 & 3 & 2.04454 & 0.5790 & 4.18E- -05 \\
\hline MDNS_SmediaMacro & 4 & -0.3598 & 1.0000 & 12 & 5.02396 & 0.0000 & $3.67 \mathrm{E}-05$ \\
\hline MDNS_Smacro & 2 & -2.73701 & 1.0000 & 7 & 2.574575 & 0.0952 & 2.15E-05 \\
\hline \multicolumn{8}{|l|}{$p$-value: 0.158} \\
\hline \multicolumn{8}{|c|}{ Maturidade de 9 meses } \\
\hline Modelos & Rank_M & $v_{-} M$ & MCS_M & Rank_R & $v \_R$ & MCS_R & Loss \\
\hline MDNS & 7 & 0.676891 & 0.9472 & 4 & 1.869714 & 0.5468 & $6.29 \mathrm{E}-05$ \\
\hline MDNS_M & 6 & 0.06783 & 1.0000 & 3 & 1.84017 & 0.5738 & $5.51 \mathrm{E}-05$ \\
\hline MDNS_P & 11 & 1.418628 & 0.4940 & 8 & 2.467554 & 0.1334 & 7.16E-05 \\
\hline MDNS_Lambda & 10 & 1.416811 & 0.4956 & 7 & 2.24912 & 0.2386 & $8.44 E-05$ \\
\hline MDNS_Macro & 1 & -3.37093 & 1.0000 & 1 & -1.72753 & 1.0000 & $2.28 \mathrm{E}-05$ \\
\hline MDNS_MMacro & 5 & -1.26091 & 1.0000 & 9 & 2.515126 & 0.1158 & $3.71 \mathrm{E}-05$ \\
\hline MDNS_PMacro & 8 & 0.955451 & 0.8240 & 11 & 3.526559 & 0.0006 & $7.02 \mathrm{E}-05$ \\
\hline MDNS_MMacroEnd & 3 & -1.87368 & 1.0000 & 6 & 2.096065 & 0.3386 & $3.71 \mathrm{E}-05$ \\
\hline MDNS_PMacroEnd & 12 & 1.468192 & 0.4584 & 12 & 3.826874 & 0.0000 & $7.13 \mathrm{E}-05$ \\
\hline MDNS_S & 9 & 0.989855 & 0.8000 & 5 & 2.083738 & 0.3504 & $6.65 \mathrm{E}-05$ \\
\hline
\end{tabular}

p-value: 0.2

Maturidade de 6 meses

\begin{tabular}{llllllll}
\multicolumn{8}{c}{ Maturidade de 6 meses } \\
\hline Modelos & Rank_M & v_M & MCS_M & Rank_R & v_R & MCS_R & Loss \\
\hline
\end{tabular}

\begin{tabular}{llllllll} 
& & & & \\
MDNS & 7 & 0.874507 & 0.8906 & 3 & 2.370503 & 0.2190 & $5.85 \mathrm{E}-05$ \\
\hline
\end{tabular}

$\begin{array}{llllllll}\text { MDNS_M } & 6 & 0.118688 & 1.0000 & 5 & 2.427126 & 0.1890 & 5.00 E-05\end{array}$

MDNS_P

MDNS_Lambda

MDNS_MMacro

MDNS PMacro

MDNS_MMacroEnd

MDNS_PMacroEnd

MDNS_S

$\begin{array}{llllccccc} & 8 & 0.946851 & 0.8572 & 6 & 2.431248 & 0.1874 & 5.91 E-05 \\ \text { MDNS_SmediaMacro } & 4 & -1.30248 & 1.0000 & 10 & 3.325037 & 0.0040 & 3.68 \mathrm{E}-05\end{array}$

MDNS Smacro

p-value: 0.4096

$\begin{array}{cccccccc}11 & 1.199577 & 0.6864 & 7 & 2.708628 & 0.0798 & 6.16 \mathrm{E}-05 \\ 9 & 1.084063 & 0.7714 & 2 & 2.213131 & 0.3194 & 6.63 \mathrm{E}-05\end{array}$

$\begin{array}{llllll}-4.46632 & 1.0000 & 1 & -2.21313 & 1.0000 & 1.72 \mathrm{E}-05\end{array}$

$\begin{array}{llllll}-1.37339 & 1.0000 & 9 & 3.106764 & 0.0138 & 3.48 \mathrm{E}-05\end{array}$

$\begin{array}{lllllll}1.57971 & 0.4096 & 12 & 4.298244 & 0.0000 & 7.31 \mathrm{E}-05\end{array}$

$\begin{array}{ccccccc}-0.94071 & 1.0000 & 8 & 2.772426 & 0.0626 & 3.93 \mathrm{E}-05 & \text { MDNS_MMacroEnd } \\ 1.093649 & 0.7640 & 11 & 3.820677 & 0.0000 & 6.16 \mathrm{E}-05 & \text { MDNS_PMacroEnd }\end{array}$

$\begin{array}{llllllll}0.946851 & 0.8572 & 6 & 2.431248 & 0.1874 & 5.91 E-05 & \text { MDNS_S }\end{array}$

MDNS_Smacro

\begin{tabular}{|c|c|c|c|c|c|c|c|}
\hline Modelos & Rank_M & v_M & MCS_M & Rank_R & $V \_R$ & MCS_R & Loss \\
\hline MDNS & 7 & 0.616186 & 0.9626 & 5 & 1.826715 & 0.5760 & 6.44E-05 \\
\hline MDNS_M & 6 & -0.08425 & 1.0000 & 4 & 1.736881 & 0.6524 & 5.61E-05 \\
\hline MDNS_P & 11 & 1.637625 & 0.3436 & 10 & 2.701003 & 0.0608 & 7.46E-05 \\
\hline MDNS_Lambda & 10 & 1.618034 & 0.3560 & 9 & 2.626653 & 0.0806 & $9.25 \mathrm{E}-05$ \\
\hline MDNS_Macro & 1 & -3.37201 & 1.0000 & 1 & -1.54914 & 1.0000 & 2.69E- 05 \\
\hline MDNS_MMacro & 5 & -1.19201 & 1.0000 & 7 & 2.140583 & 0.3068 & $4.03 E-05$ \\
\hline MDNS_PMacro & 8 & 0.673774 & 0.9464 & 11 & 3.137691 & 0.0070 & $6.74 E-05$ \\
\hline MDNS_MMacroEnd & 3 & -2.58819 & 1.0000 & 2 & 1.549138 & 0.7982 & $3.74 \mathrm{E}-05$ \\
\hline MDNS_PMacroEnd & 12 & 1.694637 & 0.3084 & 12 & 4.082039 & 0.0000 & 7.52E-05 \\
\hline MDNS_S & 9 & 1.055474 & 0.7712 & 8 & 2.510495 & 0.1202 & $6.85 \mathrm{E}-05$ \\
\hline MDNS_SmediaMacro & 4 & -1.24888 & 1.0000 & 6 & 1.937051 & 0.4726 & 4.11E-05 \\
\hline MDNS_Smacro & 2 & -3.33189 & 1.0000 & 3 & 1.679284 & 0.7982 & $3.79 \mathrm{E}-05$ \\
\hline
\end{tabular}

p-value: 0.4584

\begin{tabular}{llclllll}
\multicolumn{8}{c}{ Maturidade de 15 meses } \\
\hline Modelos & Rank_M & V_M & MCS_M & Rank_R & V_R & MCS_R & Loss \\
\hline
\end{tabular}

\begin{tabular}{lll}
2.083738 & 0.3504 & $6.65 \mathrm{E}-05$ \\
2.525674 & 0.1092 & $3.89 \mathrm{E}-05$ \\
\hline .727534 & 1.0000 & $3.50 \mathrm{E}-05$ \\
\hline
\end{tabular}

$\begin{array}{llll} & 7 & 0.347002 & 0.9974\end{array}$

MDNS_M

MDNS_Lambda

MDNS_Macro

MDNS_MMacro

MDNS_MMacroEnd

MDNS_PMacroEnd

MDNS_S

MDNS_Smacro

p-value: 0.3084

MDNS_Smacro

\begin{tabular}{|c|c|c|c|c|c|c|c|}
\hline \multicolumn{8}{|c|}{ Maturidade de 18 meses } \\
\hline Modelos & Rank_M & v_M & MCS_M & Rank_R & $v_{-} R$ & MCS_R & Loss \\
\hline MDNS & 7 & 0.256921 & 0.9998 & 6 & 1.65439 & 0.7270 & $6.07 \mathrm{E}-05$ \\
\hline MDNS_M & 6 & -0.08425 & 1.0000 & 4 & 1.62622 & 0.7476 & $5.73 \mathrm{E}-05$ \\
\hline MDNS_P & 11 & 1.776413 & 0.2716 & 9 & 2.95743 & 0.0226 & $7.31 E-05$ \\
\hline MDNS_Lambda & 12 & 1.77886 & 0.2702 & 11 & 2.985788 & 0.0202 & $9.47 \mathrm{E}-05$ \\
\hline MDNS_Macro & 1 & -3.64506 & 1.0000 & 1 & -0.9743 & 1.0000 & $3.34 \mathrm{E}-05$ \\
\hline MDNS_MMacro & 5 & -0.93253 & 1.0000 & 5 & 1.630986 & 0.7442 & 4.56E-05 \\
\hline MDNS_PMacro & 8 & 0.424895 & 0.9962 & 8 & 2.843668 & 0.0368 & $6.33 \mathrm{E}-05$ \\
\hline MDNS_MMacroEnd & 3 & -2.37456 & 1.0000 & 2 & 0.974298 & 0.9930 & 4.06E-05 \\
\hline MDNS_PMacroEnd & 10 & 1.740779 & 0.2904 & 12 & 4.630592 & 0.0000 & $7.38 \mathrm{E}-05$ \\
\hline MDNS_S & 9 & 1.058274 & 0.7710 & 10 & 2.979986 & 0.0210 & $6.80 E-05$ \\
\hline MDNS_SmediaMacro & 4 & -1.04229 & 1.0000 & 3 & 1.445798 & 0.9930 & 4.52E-05 \\
\hline MDNS_Smacro & 2 & -3.33927 & 1.0000 & 7 & 1.999826 & 0.4374 & 4.11E-05 \\
\hline
\end{tabular}

Maturidade de 21 meses

\begin{tabular}{lccccccc}
\hline Modelos & Rank_M & V_M & MCS_M & Rank_R & V_R & MCS_R & Loss \\
\hline MDNS & 7 & 0.17735 & 1.0000 & 5 & 1.812638 & 0.6346 & $5.89 \mathrm{E}-05$
\end{tabular}

$\begin{array}{llll}\text { MDNS_M } & 6 & 0.087308 & 1.0000\end{array}$

MDNS_P

MDNS_Lambda

MDNS_Macro

MDNS_MMacro

MDNS_PMacro
MDNS_MMacroEnd

MDNS_PMacroEnd

MDNS_S

MDNS_SmediaMacro

$\begin{array}{ll}1.970531 & 0.1712 \\ 1.952967 & 0.1806\end{array}$

$\begin{array}{ll}1.952967 & 0.1806\end{array}$

$\begin{array}{ll}-4.21145 & 1.0000 \\ -0.97913 & 1.0000\end{array}$

$\begin{array}{lll}-0.97913 & 1.0000\end{array}$

$0.444717 \quad 0.9980$

$-2.05221 \quad 1.0000$

$\begin{array}{ll}1.693097 & 0.3242 \\ 1.148773 & 0.7330\end{array}$

$\begin{array}{ll}-0.99314 & 1.0000 \\ -3.52595 & 1.0000\end{array}$

$\begin{array}{lll}1.676462 & 0.7216 & 6.09 \mathrm{E}-05\end{array}$

$\begin{array}{lll}1.635016 & 0.7500 & 5.57 \mathrm{E}-05\end{array}$

$\begin{array}{lll}1.635016 & 0.7500 & 5.57 \mathrm{E}-05 \\ 2.800662 & 0.0436 & 7.36 \mathrm{E}-05\end{array}$

$\begin{array}{lll}2.800662 & 0.0436 & 7.36 \mathrm{E}-05 \\ 2.806603 & 0.0418 & 9.41 \mathrm{E}-05\end{array}$

$\begin{array}{ccc}2.806603 & 0.0418 & 9.41 \mathrm{E}-0.5 \\ -1.32873 & 1.0000 & 2.97 \mathrm{E}-05\end{array}$

$\begin{array}{lll}1.877454 & 0.5324 & 4.26 \mathrm{E}-05\end{array}$

$\begin{array}{lll}2.942527 & 0.0218 & 6.47 \mathrm{E}-05\end{array}$

$\begin{array}{lll}1.328735 & 0.9306 & 3.86 \mathrm{E}-05\end{array}$ \begin{tabular}{llllllll} 
MDNS_SmediaMacro & 4 & -0.99314 & 1.0000 & 3 & 1.192629 & 1.0000 & $4.63 \mathrm{E}-05$ \\
MDNS_Smacro & 2 & -3.52595 & 1.0000 & 7 & 1.975247 & 0.4952 & $4.17 \mathrm{E}-05$ \\
\hline p-value: 0.1712 & & & & & & &
\end{tabular}

\begin{tabular}{|c|c|c|c|c|c|c|c|c|c|c|c|c|c|c|c|}
\hline \multicolumn{8}{|c|}{ Maturidade de 24 meses } & \multicolumn{8}{|c|}{ Maturidade de 27 meses } \\
\hline Modelos & Rank_M & $v_{-} M$ & MCS_M & Rank_R & $v \_R$ & MCS_R & Loss & Modelos & Rank_M & v_M & MCS_M & Rank_R & $V \_R$ & MCS $\_R$ & Loss \\
\hline MDNS & 6 & 0.052106 & 1.0000 & 6 & 1.624032 & 0.7768 & $5.60 \mathrm{E}-05$ & MDNS & 6 & 0.008126 & 1.0000 & 6 & 1.17406 & 0.9666 & $5.59 \mathrm{E}-05$ \\
\hline MDNS_M & 7 & 0.138654 & 1.0000 & 7 & 1.693505 & 0.7276 & $5.67 \mathrm{E}-05$ & MDNS_M & 7 & 0.209839 & 1.0000 & 7 & 1.306956 & 0.9220 & $5.77 E-05$ \\
\hline MDNS_P & 11 & 1.8435 & 0.2366 & 9 & 3.136385 & 0.0080 & $6.68 \mathrm{E}-05$ & MDNS_P & 11 & 1.440431 & 0.5124 & 8 & 2.366479 & 0.1856 & $6.56 \mathrm{E}-05$ \\
\hline MDNS_Lambda & 12 & 1.85605 & 0.2296 & 10 & 3.151455 & 0.0074 & $8.49 \mathrm{E}-05$ & MDNS_Lambda & 12 & 1.520814 & 0.4464 & 10 & 2.849489 & 0.0362 & $8.14 \mathrm{E}-05$ \\
\hline MDNS_Macro & 1 & -4.17332 & 1.0000 & 1 & -0.30478 & 1.0000 & $3.77 \mathrm{E}-05$ & MDNS_Macro & 1 & -3.21959 & 1.0000 & 1 & -0.06216 & 1.0000 & 4.06E-05 \\
\hline MDNS_MMacro & 4 & -0.82936 & 1.0000 & 5 & 1.390361 & 0.9116 & 4.71E-05 & MDNS_MMacro & 5 & -0.60148 & 1.0000 & 5 & 1.07377 & 0.9816 & $4.87 E-05$ \\
\hline MDNS_PMacro & 8 & 0.451705 & 0.9966 & 8 & 2.828707 & 0.0402 & $5.94 \mathrm{E}-05$ & MDNS_PMacro & 8 & 0.393157 & 0.9974 & 9 & 2.498399 & 0.1286 & $5.93 \mathrm{E}-05$ \\
\hline MDNS_MMacroEnd & 3 & -1.86384 & 1.0000 & 2 & 0.304785 & 1.0000 & 4.03E-05 & MDNS_MMacroEnd & 3 & -1.83945 & 1.0000 & 2 & 0.062156 & 1.0000 & 4.11E-05 \\
\hline MDNS_PMacroEnd & 10 & 1.525052 & 0.4570 & 12 & 4.701436 & 0.0000 & $6.66 \mathrm{E}-05$ & MDNS_PMacroEnd & 10 & 1.129286 & 0.7426 & 11 & 4.298423 & 0.0000 & $6.52 \mathrm{E}-05$ \\
\hline MDNS_S & 9 & 1.078705 & 0.7926 & 11 & 3.510614 & 0.0004 & $6.35 \mathrm{E}-05$ & MDNS_S & 9 & 0.846296 & 0.9052 & 12 & 4.369816 & 0.0000 & $6.32 \mathrm{E}-05$ \\
\hline MDNS_SmediaMacro & 5 & -0.8119 & 1.0000 & 3 & 1.116977 & 1.0000 & 4. $68 \mathrm{E}-05$ & MDNS_SmediaMacro & 4 & -0.64098 & 1.0000 & 4 & 1.052582 & 1.0000 & 4.86E-05 \\
\hline MDNS_Smacro & 2 & -3.07383 & 1.0000 & 4 & 1.351094 & 1.0000 & 4. $14 \mathrm{E}-05$ & MDNS_Smacro & 2 & -2.34619 & 1.0000 & 3 & 0.765456 & 1.0000 & 4.28E-05 \\
\hline
\end{tabular}

p-value: 0.2296

p-value 0.4464 


\begin{tabular}{|c|c|c|c|c|c|c|c|c|c|c|c|c|c|c|c|}
\hline \multicolumn{8}{|c|}{ Maturidade de 30 meses } & \multicolumn{8}{|c|}{ Maturidade de 33 meses } \\
\hline Modelos & Rank_M & $v_{-} \mathrm{M}$ & MCS_M & Rank_R & $v_{-} R$ & MCS_R & Loss & Modelos & Rank_M & $v_{-} \mathrm{M}$ & MCS_M & Rank_R & $V \_R$ & MCS_R & Loss \\
\hline MDNS & 6 & 0.011701 & 1.0000 & 6 & 1.098268 & 0.9800 & $5.56 \mathrm{E}-05$ & MDNS & 6 & 0.027187 & 1.0000 & 6 & 1.132365 & 0.9732 & $5.40 \mathrm{E}-05$ \\
\hline MDNS_M & 7 & 0.224252 & 1.0000 & 7 & 1.273797 & 0.9370 & $5.76 \mathrm{E}-05$ & MDNS_M & 7 & 0.329242 & 0.9996 & 7 & 1.731269 & 0.6370 & $5.68 \mathrm{E}-05$ \\
\hline MDNS_P & 11 & 1.283256 & 0.6060 & 8 & 2.008943 & 0.4104 & $6.42 \mathrm{E}-05$ & MDNS_P & 11 & 1.119672 & 0.7494 & 8 & 1.929463 & 0.4664 & $6.12 \mathrm{E}-05$ \\
\hline MDNS_Lambda & 12 & 1.292885 & 0.5978 & 9 & 2.616234 & 0.0738 & $7.74 \mathrm{E}-05$ & MDNS_Lambda & 12 & 1.124184 & 0.7458 & 9 & 2.349794 & 0.1800 & 7.17E-05 \\
\hline MDNS_Macro & 1 & -2.56829 & 1.0000 & 2 & 0.201654 & 1.0000 & $4.26 \mathrm{E}-05$ & MDNS_Macro & 1 & -2.143 & 1.0000 & 2 & 0.422983 & 1.0000 & 4.32E-05 \\
\hline MDNS_MMacro & 5 & -0.47023 & 1.0000 & 4 & 0.892375 & 0.9964 & $4.97 \mathrm{E}-05$ & MDNS_MMacro & 5 & -0.425 & 1.0000 & 4 & 0.784947 & 0.9994 & $4.87 E-05$ \\
\hline MDNS_PMacro & 8 & 0.432385 & 0.9962 & 10 & 2.725766 & 0.0516 & $5.90 \mathrm{E}-05$ & MDNS_PMacro & 8 & 0.462397 & 0.9968 & 10 & 2.710672 & 0.0542 & $5.72 \mathrm{E}-05$ \\
\hline MDNS_MMacroEnd & 2 & -1.96698 & 1.0000 & 1 & -0.20165 & 1.0000 & 4.09E-05 & MDNS_MMacroEnd & 2 & -1.96949 & 1.0000 & 1 & -0.42298 & 1.0000 & $3.97 E-05$ \\
\hline MDNS_PMacroEnd & 10 & 0.863734 & 0.8990 & 11 & 3.891034 & 0.0000 & $6.33 \mathrm{E}-05$ & MDNS_PMacroEnd & 10 & 0.709951 & 0.9636 & 11 & 3.183142 & 0.0058 & $6.04 \mathrm{E}-05$ \\
\hline MDNS_S & 9 & 0.743861 & 0.9450 & 12 & 4.204101 & 0.0000 & $6.26 \mathrm{E}-05$ & MDNS_S & 9 & 0.69029 & 0.9676 & 12 & 3.587647 & 0.0004 & $6.02 E-05$ \\
\hline MDNS_SmediaMacro & 4 & -0.51693 & 1.0000 & 5 & 0.918363 & 0.9964 & 4.94E-05 & MDNS_SmediaMacro & 4 & -0.44526 & 1.0000 & 5 & 0.7978 & 0.9994 & $4.87 E-05$ \\
\hline MDNS_Smacro & 3 & -1.96206 & 1.0000 & 3 & 0.420452 & 1.0000 & $4.38 \mathrm{E}-05$ & MDNS_Smacro & 3 & -1.77113 & 1.0000 & 3 & 0.513869 & 1.0000 & 4.32E-05 \\
\hline \multicolumn{8}{|l|}{ p-value: 0.5978} & \multicolumn{8}{|l|}{$p$-value: 0.7458} \\
\hline \multicolumn{8}{|c|}{ Maturidade de 36 meses } & \multicolumn{8}{|c|}{ Maturidade de 39 meses } \\
\hline Modelos & Rank_M & $v_{-} \mathrm{M}$ & MCS_M & Rank_R & $v_{-} R$ & MCS_R & Loss & Modelos & Rank_M & $v_{-} \mathrm{M}$ & MCS_M & Rank_R & $V_{-} R$ & MCS_R & Loss \\
\hline MDNS & 6 & 0.060881 & 1.0000 & 6 & 1.174094 & 0.9686 & $5.30 \mathrm{E}-05$ & MDNS & 6 & 0.117206 & 1.0000 & 6 & 1.183326 & 0.9576 & $5.32 \mathrm{E}-05$ \\
\hline MDNS_M & 7 & 0.365051 & 0.9994 & 8 & 1.904543 & 0.4930 & $5.58 \mathrm{E}-05$ & MDNS_M & 7 & 0.442807 & 0.9994 & 9 & 2.003512 & 0.4028 & 5.60E-05 \\
\hline MDNS_P & 12 & 0.966583 & 0.8594 & 7 & 1.846113 & 0.5412 & $5.89 \mathrm{E}-05$ & MDNS_P & 12 & 0.898799 & 0.9204 & 8 & 1.70387 & 0.6548 & $5.81 E-05$ \\
\hline MDNS_Lambda & 11 & 0.941176 & 0.8730 & 9 & 1.986829 & 0.4260 & $6.69 \mathrm{E}-05$ & MDNS_Lambda & 11 & 0.817253 & 0.9474 & 7 & 1.669334 & 0.6862 & $6.38 \mathrm{E}-05$ \\
\hline MDNS_Macro & 2 & -1.73967 & 1.0000 & 2 & 0.601615 & 1.0000 & 4. $38 \mathrm{E}-05$ & MDNS_Macro & 3 & -1.47966 & 1.0000 & 2 & 0.734215 & 0.9994 & $4.51 E-05$ \\
\hline MDNS_MMacro & 4 & -0.37359 & 1.0000 & 4 & 0.73566 & 0.9998 & 4.83E-05 & MDNS_MMacro & 4 & -0.36422 & 1.0000 & 4 & 0.781174 & 0.9986 & $4.84 \mathrm{E}-05$ \\
\hline MDNS_PMacro & 8 & 0.484375 & 0.9964 & 10 & 2.527004 & 0.1030 & $5.60 \mathrm{E}-05$ & MDNS_PMacro & 8 & 0.474336 & 0.9990 & 10 & 2.155728 & 0.3016 & 5.57E-05 \\
\hline MDNS_MMacroEnd & 1 & -1.92698 & 1.0000 & 1 & -0.60161 & 1.0000 & 3.86E-05 & MDNS_MMacroEnd & 1 & -1.75017 & 1.0000 & 1 & -0.73421 & 1.0000 & $3.82 \mathrm{E}-05$ \\
\hline MDNS_PMacroEnd & 9 & 0.608879 & 0.9888 & 11 & 2.67885 & 0.0560 & $5.84 \mathrm{E}-05$ & MDNS_PMacroEnd & 9 & 0.572795 & 0.9950 & 11 & 2.386981 & 0.1748 & $5.75 \mathrm{E}-05$ \\
\hline MDNS_S & 10 & 0.650517 & 0.9840 & 12 & 2.870143 & 0.0250 & $5.85 \mathrm{E}-05$ & MDNS_S & 10 & 0.676401 & 0.9832 & 12 & 2.605255 & 0.0856 & $5.81 E-05$ \\
\hline MDNS_SmediaMacro & 5 & -0.37304 & 1.0000 & 5 & 0.771441 & 0.9998 & $4.85 \mathrm{E}-05$ & MDNS_SmediaMacro & 5 & -0.32776 & 1.0000 & 5 & 0.853131 & 0.9986 & 4.90E-05 \\
\hline MDNS_Smacro & 3 & -1.56025 & 1.0000 & 3 & 0.651095 & 1.0000 & $4.34 \mathrm{E}-05$ & MDNS_Smacro & 2 & -1.51219 & 1.0000 & 3 & 0.748491 & 0.9994 & 4.40E-05 \\
\hline \multicolumn{8}{|l|}{$p$-value: 0.8594} & \multicolumn{8}{|l|}{ p-value: 0.9204} \\
\hline \multicolumn{8}{|c|}{ Maturidade de 48 meses } & \multicolumn{8}{|c|}{ Maturidade de 60 meses } \\
\hline Modelos & Rank_M & $v_{2} M$ & MCS_M & Rank_R & $v_{-} R$ & MCS_R $R$ & Loss & Modelos & Rank_M & v_M & MCS_M & Rank_R & $v_{-} R$ & MCS_R $R$ & Loss \\
\hline MDNS & 6 & 0.201215 & 1.0000 & 7 & 1.469926 & 0.8368 & $5.58 \mathrm{E}-05$ & MDNS & 7 & 0.426926 & 0.9992 & 7 & 1.709206 & 0.6956 & $6.11 \mathrm{E}-05$ \\
\hline MDNS_M & 8 & 0.438375 & 0.9998 & 12 & 1.89604 & 0.4994 & $5.79 \mathrm{E}-05$ & MDNS_M & 10 & 0.647337 & 0.9848 & 10 & 1.878661 & 0.5462 & $6.27 \mathrm{E}-05$ \\
\hline MDNS_P & 12 & 0.642482 & 0.9922 & 11 & 1.848557 & 0.5370 & $5.84 \mathrm{E}-05$ & MDNS_P & 9 & 0.530441 & 0.9958 & 9 & 1.800432 & 0.6200 & $6.14 \mathrm{E}-05$ \\
\hline MDNS_Lambda & 7 & 0.256447 & 1.0000 & 3 & 1.116193 & 0.9762 & $5.80 \mathrm{E}-05$ & MDNS_Lambda & 4 & -0.28704 & 1.0000 & 2 & 0.908794 & 0.9954 & $5.45 \mathrm{E}-05$ \\
\hline MDNS_Macro & 3 & -0.5244 & 1.0000 & 6 & 1.307234 & 0.9304 & $5.11 \mathrm{E}-05$ & MDNS_Macro & 6 & 0.144413 & 1.0000 & 8 & 1.738947 & 0.6722 & $5.89 \mathrm{E}-05$ \\
\hline MDNS_MMacro & 4 & -0.31967 & 1.0000 & 2 & 0.948395 & 0.9940 & $5.09 \mathrm{E}-05$ & MDNS_MMacro & 3 & -0.43248 & 1.0000 & 3 & 1.168635 & 0.9660 & $5.44 \mathrm{E}-05$ \\
\hline MDNS_PMacro & 10 & 0.532307 & 0.9984 & 9 & 1.777398 & 0.5982 & $5.80 \mathrm{E}-05$ & MDNS_PMacro & 11 & 0.808225 & 0.9520 & 12 & 2.023089 & 0.4322 & $6.35 E-05$ \\
\hline MDNS_MMacroEnd & 1 & -2.10883 & 1.0000 & 1 & -0.94839 & 1.0000 & $3.87 \mathrm{E}-05$ & MDNS_MMacroEnd & 1 & -2.04969 & 1.0000 & 1 & -0.90879 & 1.0000 & $4.01 E-05$ \\
\hline MDNS_PMacroEnd & 11 & 0.589785 & 0.9960 & 10 & 1.806948 & 0.5726 & $6.05 \mathrm{E}-05$ & MDNS_PMacroEnd & 12 & 0.816579 & 0.9476 & 11 & 1.959699 & 0.4816 & $6.57 \mathrm{E}-05$ \\
\hline MDNS_S & 9 & 0.500274 & 0.9990 & 8 & 1.580071 & 0.7596 & $5.87 \mathrm{E}-05$ & MDNS_S & 8 & 0.513786 & 0.9962 & 6 & 1.697509 & 0.9660 & $6.21 E-05$ \\
\hline MDNS_SmediaMacro & 5 & -0.10744 & 1.0000 & 4 & 1.118756 & 0.9762 & $5.31 \mathrm{E}-05$ & MDNS_SmediaMacro & 5 & 0.00654 & 1.0000 & 4 & 1.317479 & 0.9660 & $5.82 E-05$ \\
\hline MDNS_Smacro & 2 & -0.95099 & 1.0000 & 5 & 1.223274 & 0.9762 & $4.89 \mathrm{E}-05$ & MDNS_Smacro & 2 & -0.59219 & 1.0000 & 5 & 1.55961 & 0.9660 & $5.52 E-05$ \\
\hline \multicolumn{8}{|l|}{$p$-value: 0.9922} & \multirow{2}{*}{\multicolumn{8}{|c|}{ p-value: 0.9476}} \\
\hline & & Maturid & de de $72 r$ & eeses & & & & & & & & & & & \\
\hline Modelos & Rank_M & $v_{-} M$ & MCS_M & Rank_R & $v_{-} R$ & MCS_R $R$ & Loss & & & & & & & & \\
\hline MDNS & 9 & 0.634747 & 0.9908 & 8 & 1.920663 & 0.5288 & $6.50 \mathrm{E}-05$ & & & & & & & & \\
\hline MDNS_M & 11 & 0.756404 & 0.9698 & 10 & 2.024311 & 0.4378 & $6.60 \mathrm{E}-05$ & & & & & & & & \\
\hline MDNS_P & 6 & 0.457105 & 0.9994 & 7 & 1.852919 & 0.5820 & $6.34 \mathrm{E}-05$ & & & & & & & & \\
\hline MDNS_Lambda & 3 & -0.55634 & 1.0000 & 2 & 0.742124 & 0.9994 & $5.31 \mathrm{E}-05$ & & & & & & & & \\
\hline MDNS_Macro & 7 & 0.518012 & 0.9974 & 11 & 2.078563 & 0.3898 & $6.37 \mathrm{E}-05$ & & & & & & & & \\
\hline MDNS_MMacro & 2 & -0.57166 & 1.0000 & 3 & 1.252669 & 0.9444 & $5.57 \mathrm{E}-05$ & & & & & & & & \\
\hline MDNS_PMacro & 12 & 0.876252 & 0.9308 & 12 & 2.177397 & 0.3170 & 6.66E-05 & & & & & & & & \\
\hline MDNS_MMacroEnd & 1 & -2.21118 & 1.0000 & 1 & -0.74212 & 1.0000 & 4.10E-05 & & & & & & & & \\
\hline MDNS_PMacroEnd & 10 & 0.720006 & 0.9780 & 9 & 1.991148 & 0.4668 & $6.76 \mathrm{E}-05$ & & & & & & & & \\
\hline MDNS_S & 8 & 0.542596 & 0.9966 & 6 & 1.80764 & 0.9444 & $6.48 \mathrm{E}-05$ & & & & & & & & \\
\hline MDNS_SmediaMacro & 5 & -0.00971 & 1.0000 & 4 & 1.420119 & 0.9444 & $6.05 \mathrm{E}-05$ & & & & & & & & \\
\hline MDNS_Smacro & 4 & -0.2344 & 1.0000 & 5 & 1.791302 & 0.9444 & $5.94 \mathrm{E}-05$ & & & & & & & & \\
\hline
\end{tabular}

p-value: 0.9308

*Modelos não reportandos nas tabelas foram eliminados pelo teste. 
Figura 19 - Model Confidence Set - Horizonte de Previsão 12 meses*

\begin{tabular}{|c|c|c|c|c|c|c|c|c|c|c|c|c|c|c|c|}
\hline \multicolumn{8}{|c|}{ Maturidade de 1 mês } & \multicolumn{8}{|c|}{ Maturidade de 3 meses } \\
\hline Modelos & Rank M & $\mathrm{vM}$ & MCS M & Rank $\mathrm{R}$ & $\vee R$ & MCS R & Loss & Modelos & Rank M & $\vee \mathrm{M}$ & MCS M & Rank $\mathrm{R}$ & $\vee \mathrm{R}$ & MCS R & Loss \\
\hline MDNS & 1 & -2.05396 & 1.0000 & 2 & 0.115393 & 1.0000 & $1.81 \mathrm{E}-04$ & MDNS & 3 & -1.91438 & 1.0000 & 3 & 0.684368 & 0.9998 & $2.12 E-04$ \\
\hline MDNS_M & 4 & -1.79349 & 1.0000 & 7 & 1.235647 & 0.9406 & $2.17 \mathrm{E}-04$ & MDNS_M & 7 & -1.699 & 1.0000 & 7 & 1.006308 & 0.9852 & $2.38 \mathrm{E}-04$ \\
\hline MDNS_P & 8 & -0.78929 & 1.0000 & 9 & 1.539386 & 0.8048 & $3.42 \mathrm{E}-04$ & MDNS_P & 8 & -0.64048 & 1.0000 & 8 & 1.404412 & 0.8664 & $3.58 E-04$ \\
\hline MDNS_Lambda & 2 & -1.97933 & 1.0000 & 3 & 0.181323 & 1.0000 & $1.86 \mathrm{E}-04$ & MDNS_Lambda & 6 & -1.76287 & 1.0000 & 6 & 0.815743 & 0.9968 & $2.31 \mathrm{E}-04$ \\
\hline MDNS_Macro & 6 & -1.513 & 1.0000 & 4 & 0.628533 & 0.9998 & $2.30 \mathrm{E}-04$ & MDNS_Macro & 5 & -1.79278 & 1.0000 & 2 & 0.210579 & 1.0000 & 1.93E-04 \\
\hline MDNS_MMacro & 11 & 1.381387 & 0.3882 & 8 & 1.448483 & 0.8574 & $2.50 \mathrm{E}-03$ & MDNS_MMacro & 11 & 1.358323 & 0.4036 & 9 & 1.444627 & 0.8460 & 2.25E-03 \\
\hline MDNS_Pmacro & 3 & -1.87793 & 1.0000 & 1 & -0.11539 & 1.0000 & $1.74 \mathrm{E}-04$ & MDNS_Pmacro & 2 & -1.9177 & 1.0000 & 1 & -0.21058 & 1.0000 & 1.79E-04 \\
\hline MDNS_MMacroEnd & 10 & 0.420639 & 0.9514 & 11 & 3.818042 & 0.0086 & $5.80 \mathrm{E}-04$ & MDNS_MMacroEnd & 10 & 0.648616 & 0.8472 & 11 & 3.601621 & 0.0116 & 5.97E-04 \\
\hline MDNS_PmacroEnd & 5 & -1.66064 & 1.0000 & 6 & 1.145639 & 0.9642 & $3.01 E-04$ & MDNS_PmacroEnd & 1 & -2.43727 & 1.0000 & 5 & 0.800959 & 0.9970 & 2.57E-04 \\
\hline MDNS_S & 9 & -0.06089 & 1.0000 & 10 & 1.774018 & 0.6416 & 4.87E-04 & MDNS_S & 9 & -0.02798 & 1.0000 & 10 & 1.681605 & 0.6942 & $4.71 \mathrm{E}-04$ \\
\hline MDNS_Smacro & 7 & -1.48688 & 1.0000 & 5 & 1.052234 & 0.9832 & $2.90 \mathrm{E}-04$ & MDNS_Smacro & 4 & -1.84377 & 1.0000 & 4 & 0.692617 & 0.9996 & $2.56 \mathrm{E}-04$ \\
\hline \multicolumn{8}{|l|}{ p-value: 0.3882} & \multicolumn{8}{|l|}{ p-value: 0.4036} \\
\hline \multicolumn{8}{|c|}{ Maturidade de 6 meses } & & & Maturid & ade de 9 & eses & & & \\
\hline Modelos & Rank M & $\vee \mathrm{M}$ & MCS M & Rank $\mathrm{R}$ & $\vee R$ & MCS R & Loss & Modelos & Rank M & $\vee \mathrm{M}$ & MCS M & Rank $\mathrm{R}$ & $\vee R$ & MCS R & Loss \\
\hline MDNS & 2 & -1.95796 & 1.0000 & 3 & 0.333371 & 1.0000 & $2.38 \mathrm{E}-04$ & MDNS & 2 & -2.1167 & 1.0000 & 3 & 0.039484 & 1.0000 & 2.63E-04 \\
\hline MDNS_M & 7 & -1.65368 & 1.0000 & 7 & 0.895278 & 0.9934 & $2.71 \mathrm{E}-04$ & MDNS_M & 5 & -1.79171 & 1.0000 & 5 & 0.841198 & 0.9968 & 2.95E-04 \\
\hline MDNS_P & 8 & -0.55011 & 1.0000 & 8 & 1.216921 & 0.9448 & $3.82 E-04$ & MDNS_P & 8 & -0.57989 & 1.0000 & 8 & 1.206651 & 0.9388 & $4.01 \mathrm{E}-04$ \\
\hline MDNS_Lambda & 4 & -1.83217 & 1.0000 & 5 & 0.578786 & 1.0000 & $2.52 \mathrm{E}-04$ & MDNS_Lambda & 4 & -1.89704 & 1.0000 & 7 & 1.066794 & 0.9746 & 2.87E-04 \\
\hline MDNS_Macro & 5 & -1.75189 & 1.0000 & 1 & -0.25407 & 1.0000 & $2.10 E-04$ & MDNS_Macro & 7 & -1.61354 & 1.0000 & 2 & 0.013807 & 1.0000 & 2.61E-04 \\
\hline MDNS_MMacro & 11 & 1.371346 & 0.4034 & 9 & 1.45707 & 0.8394 & $2.08 \mathrm{E}-03$ & MDNS_MMacro & 11 & 1.412771 & 0.3976 & 9 & 1.540238 & 0.7886 & 1.95E-03 \\
\hline MDNS_Pmacro & 6 & -1.7376 & 1.0000 & 2 & 0.254072 & 1.0000 & $2.28 \mathrm{E}-04$ & MDNS_Pmacro & 6 & -1.62033 & 1.0000 & 4 & 0.40685 & 1.0000 & $2.85 E-04$ \\
\hline MDNS_MMacroEnd & 10 & 0.693939 & 0.8410 & 11 & 3.304394 & 0.0238 & $6.02 \mathrm{E}-04$ & MDNS_MMacroEnd & 10 & 0.913956 & 0.7234 & 11 & 3.41595 & 0.0174 & $6.46 E-04$ \\
\hline MDNS_PmacroEnd & 1 & -2.15733 & 1.0000 & 6 & 0.681957 & 0.9996 & $2.82 \mathrm{E}-04$ & MDNS_PmacroEnd & 3 & -1.93218 & 1.0000 & 6 & 0.920432 & 0.9922 & $3.31 \mathrm{E}-04$ \\
\hline MDNS_S & 9 & -0.03971 & 1.0000 & 10 & 1.715579 & 0.6624 & $4.74 \mathrm{E}-04$ & MDNS_S & 9 & -0.14846 & 1.0000 & 10 & 1.704684 & 0.6750 & 4.74E-04 \\
\hline MDNS_Smacro & 3 & -1.92233 & 1.0000 & 4 & 0.394772 & 1.0000 & $2.63 \mathrm{E}-04$ & MDNS_Smacro & 1 & -2.23027 & 1.0000 & 1 & -0.01381 & 1.0000 & 2.6OE-04 \\
\hline$p$-value: 0.4034 & & & & & & & & $p$-value: 0.3976 & & & & & & & \\
\hline & & Maturid & ade de 12 & neses & & & & & & Maturid & ade de 15 & neses & & & \\
\hline Modelos & Rank M & $\vee M$ & MCS M & Rank R & $\vee R$ & MCS R & Loss & Modelos & Rank M & $\vee M$ & MCS M & Rank R & $\vee R$ & MCS R & Loss \\
\hline MDNS & 1 & -3.08256 & 1.0000 & 2 & 0.333265 & 1.0000 & $2.83 \mathrm{E}-04$ & MDNS & 1 & -3.18302 & 1.0000 & 2 & 0.657737 & 0.9998 & $2.97 E-04$ \\
\hline MDNS_M & 5 & -2.54762 & 1.0000 & 4 & 0.632205 & 0.9998 & 3.10E-04 & MDNS_M & 4 & -2.74145 & 1.0000 & 4 & 0.769292 & 0.9986 & 3.20E-04 \\
\hline MDNS_P & 8 & -0.94422 & 1.0000 & 6 & 1.065869 & 0.9826 & 4.10E-04 & MDNS_P & 8 & -1.06067 & 1.0000 & 5 & 1.1184 & 0.9748 & 4.12E-04 \\
\hline MDNS_Lambda & 3 & -2.80814 & 1.0000 & 8 & 1.448998 & 0.8570 & $3.15 E-04$ & MDNS_Lambda & 3 & -2.83345 & 1.0000 & 7 & 1.645781 & 0.7298 & 3.33E-04 \\
\hline MDNS_Macro & 7 & -1.83887 & 1.0000 & 3 & 0.372953 & 1.0000 & $3.12 \mathrm{E}-04$ & MDNS_Macro & 7 & -1.58805 & 1.0000 & 3 & 0.717397 & 0.9998 & 3.55E-04 \\
\hline MDNS_MMacro & 11 & 1.304843 & 0.5598 & 9 & 1.569821 & 0.7854 & $1.83 \mathrm{E}-03$ & MDNS_MMacro & 11 & 1.361065 & 0.5334 & 8 & 1.688395 & 0.7016 & $1.71 \mathrm{E}-03$ \\
\hline MDNS_PMacro & 6 & -1.98653 & 1.0000 & 5 & 0.80647 & 0.9988 & $3.33 \mathrm{E}-04$ & MDNS_PMacro & 6 & -1.78413 & 1.0000 & 6 & 1.193202 & 0.9576 & 3.73E-04 \\
\hline MDNS_MMacroEnd & 10 & 0.706795 & 0.9280 & 12 & 3.394994 & 0.0222 & $6.88 \mathrm{E}-04$ & MDNS_MMacroEnd & 10 & 0.982816 & 0.7868 & 12 & 3.539777 & 0.0132 & 7.20E-04 \\
\hline MDNS_PMacroEnd & 4 & -2.58676 & 1.0000 & 7 & 1.377958 & 0.8948 & $3.73 E-04$ & MDNS_PMacroEnd & 5 & -2.32986 & 1.0000 & 10 & 1.910399 & 0.5466 & 4.05E-04 \\
\hline MDNS_S & 9 & -0.8539 & 1.0000 & 10 & 1.736916 & 0.6676 & 4.68E-04 & MDNS_S & 9 & -0.97854 & 1.0000 & 9 & 1.783929 & 0.6376 & 4.61E-04 \\
\hline MDNS_SmediaMacro & 12 & 1.935719 & 0.2036 & 11 & 3.096328 & 0.0466 & $1.23 \mathrm{E}-03$ & MDNS_SmediaM & 12 & 1.877864 & 0.2416 & 11 & 3.101419 & 0.0448 & 1.15E-03 \\
\hline MDNS_Smacro & 2 & -2.87745 & 1.0000 & 1 & -0.33326 & 1.0000 & $2.49 \mathrm{E}-04$ & MDNS_Smacro & 2 & -3.17072 & 1.0000 & 1 & -0.65774 & 1.0000 & $2.34 E-04$ \\
\hline$p$-value: 0.2036 & & & & & & & & p-value: 0.2416 & & & & & & & \\
\hline & & Maturid & ade de 18 & neses & & & & & & Maturid & ade de 21 & neses & & & \\
\hline Modelos & Rank M & $v M$ & MCS M & Rank R & $\vee R$ & MCS R & Loss & Modelos & Rank M & $v M$ & MCS M & Rank R & $\vee R$ & MCS R & Loss \\
\hline MDNS & 2 & -3.23963 & 1.0000 & 2 & 0.953807 & 0.9922 & $3.14 \mathrm{E}-04$ & MDNS & 2 & -3.24897 & 1.0000 & 3 & 1.193225 & 0.9592 & $3.25 E-04$ \\
\hline MDNS_M & 3 & -2.87975 & 1.0000 & 3 & 0.991923 & 0.9912 & $3.31 \mathrm{E}-04$ & MDNS_M & 3 & -3.03733 & 1.0000 & 2 & 1.173491 & 1.0000 & 3.37E-04 \\
\hline MDNS_P & 8 & -1.17268 & 1.0000 & 5 & 1.135121 & 0.9750 & $4.12 \mathrm{E}-04$ & MDNS_P & 6 & -1.34476 & 1.0000 & 5 & 1.241575 & 0.9458 & 4.06E-04 \\
\hline MDNS_Lambda & 4 & -2.84274 & 1.0000 & 7 & 1.732688 & 0.6776 & 3.50E-04 & MDNS_Lambda & 4 & -2.79554 & 1.0000 & 7 & 1.702044 & 0.6854 & 3.61E-04 \\
\hline MDNS_Macro & 7 & -1.35868 & 1.0000 & 4 & 1.007404 & 0.9896 & 3.93E-04 & MDNS_Macro & 9 & -1.14789 & 1.0000 & 4 & 1.223959 & 0.9508 & 4.21E-04 \\
\hline MDNS_MMacro & 11 & 1.387781 & 0.5344 & 8 & 1.781373 & 0.6406 & $1.61 \mathrm{E}-03$ & MDNS_MMacro & 11 & 1.414144 & 0.5154 & 8 & 1.865908 & 0.5690 & 1.52E-03 \\
\hline MDNS_PMacro & 6 & -1.51015 & 1.0000 & 6 & 1.453897 & 0.8586 & 4.08E-04 & MDNS_PMacro & 7 & -1.26723 & 1.0000 & 6 & 1.662471 & 0.7164 & 4.36E-04 \\
\hline MDNS_MMacroEnd & 10 & 1.175976 & 0.6728 & 12 & 3.579613 & 0.0106 & 7.49E-04 & MDNS_MMacroEnd & 10 & 1.368804 & 0.5458 & 12 & 3.730908 & 0.0096 & 7.70E-04 \\
\hline MDNS_PMacroEnd & 5 & -1.97614 & 1.0000 & 10 & 2.323771 & 0.2618 & $4.34 \mathrm{E}-04$ & MDNS_PMacroEnd & 5 & -1.67254 & 1.0000 & 10 & 2.67052 & 0.1278 & 4.56E-04 \\
\hline MDNS_S & 9 & -1.08702 & 1.0000 & 9 & 1.966231 & 0.5006 & $4.58 \mathrm{E}-04$ & & 8 & -1.17517 & 1.0000 & 9 & 2.092665 & 0.4124 & 4.53E-04 \\
\hline MDNS_SmediaM & 12 & 1.860954 & 0.2710 & 11 & 3.083596 & 0.0454 & $1.08 E-03$ & MDNS_SmediaM & 12 & 1.8334 & 0.2812 & 11 & 3.083989 & 0.0506 & $1.02 E-03$ \\
\hline MDNS_Smacro & 1 & -3.4363 & 1.0000 & 1 & -0.95381 & 1.0000 & $2.23 \mathrm{E}-04$ & MDNS_Smacro & 1 & -3.5835 & 1.0000 & 1 & -1.17349 & 1.0000 & $2.15 \mathrm{E}-04$ \\
\hline p-value: 0.271 & & & & & & & & $p$-value: 0.2812 & & & & & & & \\
\hline & & Maturid & ade de 24 & neses & & & & & & Maturid & ade de 27 & heses & & & \\
\hline Modelos & Rank M & $v M$ & MCS M & Rank R & $\vee R$ & MCS R & Loss & Modelos & Rank M & $\vee M$ & MCS M & Rank R & $\vee R$ & MCS R & Loss \\
\hline MDNS & 2 & -3.31576 & 1.0000 & 4 & 1.366953 & 0.8982 & 3.32E-04 & MDNS & 3 & -3.37105 & 1.0000 & 4 & 1.655816 & 0.7414 & 3.40E-04 \\
\hline MDNS_M & 3 & -3.2038 & 1.0000 & 2 & 308904 & 1.0000 & 3.40E-04 & MDNS_M & 2 & -3.43092 & 1.0000 & 3 & 1.559342 & 0.9920 & 3.44E-04 \\
\hline MDNS_P & 5 & -1.52684 & 1.0000 & 3 & 1.318541 & 0.9946 & $3.98 E-04$ & MDNS_P & 5 & -1.88976 & 1.0000 & 2 & 1.527774 & 1.0000 & $3.92 E-04$ \\
\hline MDNS_Lambda & 4 & -2.85545 & 1.0000 & 6 & 1.788194 & 0.6368 & 3.66E-04 & MDNS_Lambda & 4 & -2.83654 & 1.0000 & 7 & 2.090127 & 0.4250 & $3.72 \mathrm{E}-04$ \\
\hline MDNS_Macro & 9 & -0.96496 & 1.0000 & 5 & 1.403517 & 0.8808 & 4.43E-04 & MDNS_Macro & 9 & -0.8619 & 1.0000 & 5 & 1.730925 & 0.6886 & 4.63E-04 \\
\hline MDNS_MMacro & 10 & 1.427134 & 0.5124 & 8 & 1.94174 & 0.5244 & $1.44 \mathrm{E}-03$ & MDNS_MMacro & 10 & 1.474131 & 0.4934 & 6 & 2.031014 & 0.4654 & $1.37 E-03$ \\
\hline MDNS_PMacro & 8 & -1.03431 & 1.0000 & 7 & 1.872286 & 0.5716 & 4.59E-04 & MDNS_PMacro & 8 & -0.86414 & 1.0000 & 8 & 2.235189 & 0.3332 & 4.79E-04 \\
\hline MDNS_MMacroEnd & 11 & 1.479869 & 0.4792 & 12 & 3.697447 & 0.0096 & $7.86 \mathrm{E}-04$ & MDNS_MMacroEnd & 11 & 1.683495 & 0.3732 & 12 & 3.579449 & 0.0140 & $8.01 E-04$ \\
\hline MDNS_PMacroEnd & 6 & -1.3698 & 1.0000 & 10 & 2.873935 & 0.0686 & $4.72 E-04$ & MDNS_PMacroEnd & 7 & -1.08065 & 1.0000 & 11 & 3.249905 & 0.0318 & 4.88E-04 \\
\hline MDNS_S & 7 & -1.31518 & 1.0000 & 9 & 2.226652 & 0.3200 & $4.46 E-04$ & MDNS_S & 6 & -1.35818 & 1.0000 & 9 & 2.375128 & 0.2480 & 4.42E-04 \\
\hline MDNS_SmediaMacro & 12 & 1.792874 & 0.2956 & 11 & 3.037568 & 0.0464 & $9.69 \mathrm{E}-04$ & MDNS_SmediaMacro & 12 & 1.86168 & 0.2746 & 10 & 3.032659 & 0.0542 & $9.28 E-04$ \\
\hline MDNS_Smacro & 1 & -3.67122 & 1.0000 & 1 & -1.3089 & 1.0000 & $2.07 \mathrm{E}-04$ & MDNS_Smacro & 1 & -3.92116 & 1.0000 & 1 & -1.52777 & 1.0000 & 2.01E-04 \\
\hline
\end{tabular}




\begin{tabular}{|c|c|c|c|c|c|c|c|c|c|c|c|c|c|c|c|}
\hline \multicolumn{8}{|c|}{ Maturidade de 30 meses } & \multicolumn{8}{|c|}{ Maturidade de 33 meses } \\
\hline Modelos & Rank_M & $v \_M$ & MCS_M & Rank $R$ & $V \_R$ & MCS_R & Loss & Modelos & Rank_M & $v \_M$ & MCS_M & Rank_R & $V \_R$ & MCS_R & Loss \\
\hline MDNS & 3 & -3.38715 & 1.0000 & 4 & 1.81524 & 0.6182 & 3.44E-04 & MDNS & 3 & -3.36375 & 1.0000 & 4 & 1.922732 & 0.5358 & 3.44E-04 \\
\hline MDNS_M & 2 & -3.60493 & 1.0000 & 3 & 1.699196 & 0.9952 & $3.46 E-04$ & MDNS_M & 2 & -3.74806 & 1.0000 & 3 & 1.79106 & 0.9992 & 3.42E-04 \\
\hline MDNS_P & 5 & -2.15809 & 1.0000 & 2 & 1.629272 & 1.0000 & $3.85 E-04$ & MDNS_P & 5 & -2.43205 & 1.0000 & 2 & 1.680721 & 1.0000 & $3.73 E-04$ \\
\hline MDNS_Lambda & 4 & -2.86183 & 1.0000 & 7 & 2.237025 & 0.3322 & $3.74 E-04$ & MDNS_Lambda & 4 & -2.83064 & 1.0000 & 7 & 2.332231 & 0.2726 & $3.71 \mathrm{E}-04$ \\
\hline MDNS_Macro & 8 & -0.71018 & 1.0000 & 5 & 1.901342 & 0.5574 & $4.78 \mathrm{E}-04$ & MDNS_Macro & 8 & -0.57458 & 1.0000 & 5 & 2.020812 & 0.4678 & $4.84 \mathrm{E}-04$ \\
\hline MDNS_MMacro & 10 & 1.535879 & 0.4588 & 6 & 2.169356 & 0.3742 & $1.30 \mathrm{E}-03$ & MDNS_MMacro & 10 & 1.539542 & 0.4584 & 6 & 2.217393 & 0.3334 & $1.24 \mathrm{E}-03$ \\
\hline MDNS_PMacro & 9 & -0.64358 & 1.0000 & 8 & 2.380463 & 0.2478 & 4.94E-04 & MDNS_PMacro & 9 & -0.44866 & 1.0000 & 8 & 2.538312 & 0.1834 & $5.02 \mathrm{E}-04$ \\
\hline MDNS_MMacroEnd & 11 & 1.801113 & 0.3068 & 12 & 3.597837 & 0.0118 & $8.10 \mathrm{E}-04$ & MDNS_MMacroEnd & 11 & 1.825588 & 0.2946 & 11 & 3.427971 & 0.0188 & $8.13 E-04$ \\
\hline MDNS_PMacroEnd & 7 & -0.81688 & 1.0000 & 11 & 3.443796 & 0.0206 & 4.99E-04 & MDNS_PMacroEnd & 7 & -0.58531 & 1.0000 & 12 & 3.624253 & 0.0116 & $5.04 E-04$ \\
\hline MDNS_S & 6 & -1.48553 & 1.0000 & 9 & 2.501599 & 0.1890 & $4.36 \mathrm{E}-04$ & MDNS_S & 6 & -1.57623 & 1.0000 & 9 & 2.563353 & 0.1732 & $4.26 \mathrm{E}-04$ \\
\hline MDNS_SmediaMacro & 12 & 1.850146 & 0.2850 & 10 & 3.044066 & 0.0524 & $8.93 E-04$ & MDNS_SmediaMacro & 12 & 1.850324 & 0.2800 & 10 & 3.120936 & 0.0424 & $8.57 E-04$ \\
\hline MDNS_Smacro & 1 & -4.02113 & 1.0000 & 1 & -1.62927 & 1.0000 & $1.96 \mathrm{E}-04$ & MDNS_Smacro & 1 & -4.09731 & 1.0000 & 1 & -1.68072 & 1.0000 & $1.91 \mathrm{E}-04$ \\
\hline \multicolumn{8}{|l|}{ p-value: 0.285} & \multicolumn{8}{|l|}{ p-value: 0.28} \\
\hline \multicolumn{8}{|c|}{ Maturidade de 36 meses } & \multicolumn{8}{|c|}{ Maturidade de 39 meses } \\
\hline Modelos & Rank_M & $\mathrm{V} \_\mathrm{M}$ & MCS_M & Rank $R$ & $V \_R$ & MCS_R & Loss & Modelos & Rank_M & $v \_M$ & MCS_M & Rank_R & $\vee \_R$ & MCS_R & Loss \\
\hline MDNS & 3 & -3.39792 & 1.0000 & 4 & 1.976088 & 0.4930 & $3.42 \mathrm{E}-04$ & MDNS & 3 & -3.36668 & 1.0000 & 4 & 2.023332 & 0.4618 & $3.42 \mathrm{E}-04$ \\
\hline MDNS_M & 2 & -3.88669 & 1.0000 & 3 & 1.838815 & 0.9998 & $3.38 \mathrm{E}-04$ & MDNS_M & 2 & -3.97013 & 1.0000 & 3 & 1.885996 & 0.9998 & $3.36 \mathrm{E}-04$ \\
\hline MDNS_P & 5 & -2.69748 & 1.0000 & 2 & 1.708707 & 1.0000 & $3.63 \mathrm{E}-04$ & MDNS_P & 4 & -2.98521 & 1.0000 & 2 & 1.74406 & 1.0000 & $3.55 \mathrm{E}-04$ \\
\hline MDNS_Lambda & 4 & -2.85905 & 1.0000 & 7 & 2.378089 & 0.2492 & $3.66 \mathrm{E}-04$ & MDNS_Lambda & 5 & -2.82371 & 1.0000 & 6 & 2.380747 & 0.2430 & $3.64 \mathrm{E}-04$ \\
\hline MDNS_Macro & 7 & -0.43519 & 1.0000 & 5 & 2.08096 & 0.4202 & 4.89E-04 & MDNS_Macro & 7 & -0.3025 & 1.0000 & 5 & 2.15851 & 0.3730 & 4.95E-04 \\
\hline MDNS_MMacro & 10 & 1.563174 & 0.4466 & 6 & 2.31049 & 0.2806 & $1.18 \mathrm{E}-03$ & MDNS_MMacro & 10 & 1.597854 & 0.4230 & 7 & 2.391943 & 0.2368 & $1.13 \mathrm{E}-03$ \\
\hline MDNS_PMacro & 9 & -0.25891 & 1.0000 & 9 & 2.627727 & 0.1452 & $5.08 \mathrm{E}-04$ & MDNS_PMacro & 9 & -0.09236 & 1.0000 & 9 & 2.779514 & 0.1070 & $5.15 E-04$ \\
\hline MDNS_MMacroEnd & 12 & 1.852956 & 0.2810 & 11 & 3.361637 & 0.0262 & $8.14 E-04$ & MDNS_MMacroEnd & 12 & 1.912653 & 0.2484 & 11 & 3.323308 & 0.0288 & $8.17 \mathrm{E}-04$ \\
\hline MDNS_PMacroEnd & 8 & -0.37156 & 1.0000 & 12 & 3.713103 & 0.0102 & $5.07 \mathrm{E}-04$ & MDNS_PMacroEnd & 8 & -0.18882 & 1.0000 & 12 & 3.813107 & 0.0066 & $5.11 \mathrm{E}-04$ \\
\hline MDNS_S & 6 & -1.6965 & 1.0000 & 8 & 2.59191 & 0.1578 & 4.16E-04 & MDNS_S & 6 & -1.76283 & 1.0000 & 8 & 2.591288 & 0.1536 & $4.08 \mathrm{E}-04$ \\
\hline MDNS_SmediaMacro & 11 & 1.821467 & 0.2974 & 10 & 3.088133 & 0.0528 & $8.27 \mathrm{E}-04$ & MDNS_SmediaMacro & 11 & 1.803324 & 0.3014 & 10 & 3.098225 & 0.0482 & $8.03 E-04$ \\
\hline MDNS_Smacro & 1 & -4.13721 & 1.0000 & 1 & -1.70871 & 1.0000 & $1.87 \mathrm{E}-04$ & MDNS_Smacro & 1 & -4.10094 & 1.0000 & 1 & -1.74406 & 1.0000 & $1.84 E-04$ \\
\hline \multicolumn{8}{|l|}{ p-value: 0.281} & \multicolumn{8}{|l|}{ p-value: 0.2484} \\
\hline \multicolumn{8}{|c|}{ Maturidade de 48 meses } & \multicolumn{8}{|c|}{ Maturidade de 60 meses } \\
\hline Modelos & Rank_M & $v \_M$ & MCS_M & Rank_R & $V \_R$ & MCS_R & Loss & Modelos & Rank_M & $\mathrm{v} \_\mathrm{M}$ & MCS_M & Rank_R & $V \_R$ & MCS_R & Loss \\
\hline MDNS & 4 & -3.4205 & 1.0000 & 4 & 2.186022 & 0.3540 & $3.36 \mathrm{E}-04$ & MDNS & 4 & -3.8175 & 1.0000 & 4 & 2.666789 & 0.1440 & 3.28E-04 \\
\hline MDNS_M & 1 & -4.24157 & 1.0000 & 3 & 2.028409 & 1.0000 & $3.28 \mathrm{E}-04$ & MDNS_M & 2 & -4.80927 & 1.0000 & 3 & 2.466482 & 1.0000 & 3.19E-04 \\
\hline MDNS_P & 3 & -3.80772 & 1.0000 & 2 & 1.879023 & 1.0000 & $3.38 \mathrm{E}-04$ & MDNS_P & 1 & -5.02015 & 1.0000 & 2 & 2.307268 & 1.0000 & 3.22E-04 \\
\hline MDNS_Lambda & 5 & -2.91487 & 1.0000 & 6 & 2.473892 & 0.1952 & $3.51 \mathrm{E}-04$ & MDNS_Lambda & 5 & -3.35682 & 1.0000 & 5 & 2.90415 & 0.0814 & $3.36 \mathrm{E}-04$ \\
\hline MDNS_Macro & 7 & 0.07358 & 1.0000 & 5 & 2.381956 & 0.2382 & $5.11 \mathrm{E}-04$ & MDNS_Macro & 7 & 0.548671 & 0.9898 & 8 & 3.061487 & 0.0550 & $5.26 \mathrm{E}-04$ \\
\hline MDNS_MMacro & 10 & 1.661634 & 0.3914 & 7 & 2.610097 & 0.1470 & $1.02 \mathrm{E}-03$ & MDNS_MMacro & 10 & 1.829944 & 0.2908 & 6 & 2.989907 & 0.0654 & $9.26 \mathrm{E}-04$ \\
\hline MDNS_PMacro & 9 & 0.340667 & 0.9992 & 9 & 3.010212 & 0.0590 & $5.29 \mathrm{E}-04$ & MDNS_PMacro & 9 & 0.828028 & 0.9170 & 9 & 3.565898 & 0.0144 & $5.40 \mathrm{E}-04$ \\
\hline MDNS_MMacroEnd & 12 & 2.004702 & 0.2154 & 11 & 3.25754 & 0.0314 & $8.17 \mathrm{E}-04$ & MDNS_MMacroEnd & 12 & 2.196281 & 0.1546 & 10 & 3.587268 & 0.0136 & $8.13 E-04$ \\
\hline MDNS_PMacroEnd & 8 & 0.22335 & 1.0000 & 12 & 3.901062 & 0.0060 & $5.18 \mathrm{E}-04$ & MDNS_PMacroEnd & 8 & 0.601449 & 0.9820 & 12 & 4.562652 & 0.0014 & $5.22 \mathrm{E}-04$ \\
\hline MDNS_S & 6 & -2.14935 & 1.0000 & 8 & 2.686794 & 0.1242 & 3.86E-04 & MDNS_S & 6 & -2.62639 & 1.0000 & 7 & 3.036537 & 0.0582 & 3.63E- 04 \\
\hline MDNS_SmediaMacro & 11 & 1.752561 & 0.3422 & 10 & 3.105474 & 0.0446 & $7.52 \mathrm{E}-04$ & MDNS_SmediaMacro & 11 & 1.920447 & 0.2514 & 11 & 3.644629 & 0.0114 & $7.09 \mathrm{E}-04$ \\
\hline MDNS_Smacro & 2 & -4.10822 & 1.0000 & 1 & -1.87902 & 1.0000 & 1.73E-04 & MDNS_Smacro & 3 & -4.78683 & 1.0000 & 1 & -2.30727 & 1.0000 & 1.63E-04 \\
\hline \multicolumn{8}{|l|}{ p-value: 0.2154} & \multirow{4}{*}{\multicolumn{8}{|c|}{ p-value: 0.1546}} \\
\hline & & Maturid & ade de 72 & neses & & & & & & & & & & & \\
\hline Modelos & Rank_M & v_M & MCS_M & Rank_R & $v_{-} R$ & MCS_R & Loss & & & & & & & & \\
\hline MDNS_Smacro & 1 & -2.35066 & 1 & & -2.35066 & & 0.000161 & & & & & & & & \\
\hline
\end{tabular}

*Modelos não reportandos nas tabelas foram eliminados pelo teste. 
Figura 20 - Model Confidence Set - Horizonte de Previsão 60 meses*

\begin{tabular}{|c|c|c|c|c|c|c|c|c|c|c|c|c|c|c|c|}
\hline \multicolumn{8}{|c|}{ Maturidade de 1 mês } & \multicolumn{8}{|c|}{ Maturidade de 3 meses } \\
\hline Modelos & Rank_M & $v_{-} M$ & MCS_M & Rank_R $R$ & $V \_R$ & MCS_R & Loss & Modelos & Rank_M & V_M & MCS_M & Rank_R $R$ & $v \vee R$ & MCS_R & Loss \\
\hline MDNS & 1 & -4.51065 & 1.0000 & 1 & -0.11504 & 1.0000 & $1.01 \mathrm{E}-03$ & MDNS & 2 & -4.48417 & 1.0000 & 2 & 0.625001 & 1.0000 & $9.98 E-04$ \\
\hline MDNS_M & 2 & -4.50998 & 1.0000 & 2 & 0.11504 & 1.0000 & $1.01 E-03$ & MDNS_M & 3 & -4.48302 & 1.0000 & 3 & 0.793999 & 0.9998 & $1.01 \mathrm{E}-03$ \\
\hline MDNS_P & 4 & -4.38823 & 1.0000 & 12 & 6.620455 & 0.0002 & $4.48 \mathrm{E}-03$ & MDNS_P & 4 & -4.35795 & 1.0000 & 12 & 6.83452 & 0.0000 & $4.07 E-03$ \\
\hline MDNS_Lambda & 3 & -4.50853 & 1.0000 & 3 & 0.415125 & 1.0000 & $1.04 \mathrm{E}-03$ & MDNS_Lambda & 1 & -4.48516 & 1.0000 & 1 & -0.625 & 1.0000 & $9.65 \mathrm{E}-04$ \\
\hline MDNS_Macro & 7 & -1.37424 & 1.0000 & 6 & 1.77539 & 0.6324 & $6.20 \mathrm{E}-02$ & MDNS_Macro & 7 & -1.26952 & 1.0000 & 6 & 1.753851 & 0.6648 & $5.52 \mathrm{E}-02$ \\
\hline MDNS_MMacro & 8 & -0.50411 & 1.0000 & 10 & 2.325391 & 0.2582 & $9.45 \mathrm{E}-02$ & MDNS_MMacro & 8 & -0.38735 & 1.0000 & 9 & 2.280578 & 0.2910 & $8.46 \mathrm{E}-02$ \\
\hline MDNS_PMacro & 6 & -3.85262 & 1.0000 & 4 & 1.336648 & 0.9360 & $1.22 \mathrm{E}-02$ & MDNS_PMacro & 6 & -4.03259 & 1.0000 & 4 & 1.281259 & 0.9536 & $7.77 \mathrm{E}-03$ \\
\hline MDNS_MMacroEnd & 5 & -4.33072 & 1.0000 & 11 & 2.6481 & 0.1378 & $1.09 E-02$ & MDNS_MMacroEnd & 5 & -4.31213 & 1.0000 & 11 & 2.789207 & 0.1088 & $9.02 \mathrm{E}-03$ \\
\hline MDNS_PMacroEnd & 9 & 0.397318 & 0.9968 & 5 & 1.42012 & 0.8924 & $1.59 \mathrm{E}-01$ & MDNS_PMacroEnd & 9 & 0.07925 & 1.0000 & 5 & 1.432644 & 0.8830 & $1.06 \mathrm{E}-01$ \\
\hline MDNS_S & 11 & 1.527671 & 0.4790 & 9 & 2.313189 & 0.2626 & $2.97 \mathrm{E}-01$ & IDNS_S & 11 & 1.535995 & 0.4644 & 10 & 2.313834 & 0.2730 & $2.57 \mathrm{E}-01$ \\
\hline MDNS_SmediaMacro & 10 & 1.500627 & 0.4968 & 8 & 2.277987 & 0.2808 & $3.04 \mathrm{E}-01$ & MDNS_SmediaMacro & 10 & 1.518316 & 0.4772 & 8 & 2.241196 & 0.3112 & $2.71 \mathrm{E}-01$ \\
\hline MDNS_Smacro & 12 & 1.752431 & 0.3436 & 7 & 2.170772 & 0.3416 & 4.71E-01 & MDNS_Smacro & 12 & 1.756511 & 0.3438 & 7 & 2.150736 & 0.3654 & 4.13E-01 \\
\hline \multicolumn{8}{|l|}{ p-value: 0.3436} & \multicolumn{8}{|l|}{ p-value: 0.3438} \\
\hline & & Maturid & ade de 6 & eses & & & & & & Maturid & ade de 9 & eses & & & \\
\hline Modelos & Rank M & $v M$ & MCS M & Rank R & $\vee R$ & MCS R & Loss & Modelos & Rank M & $v M$ & MCS M & Rank $R$ & $\vee R$ & MCS R & Loss \\
\hline MDNS & 1 & -4.22484 & 1.0000 & 2 & 0.180102 & 1.0000 & $1.00 \mathrm{E}-03$ & MDNS & 1 & -4.26608 & 1.0000 & 2 & 0.092578 & 1.0000 & $9.98 E-04$ \\
\hline MDNS_M & 3 & -4.22387 & 1.0000 & 3 & 0.258448 & 1.0000 & $1.01 E-03$ & MDNS_M & 3 & -4.26494 & 1.0000 & 3 & 0.339551 & 1.0000 & $1.01 \mathrm{E}-03$ \\
\hline MDNS P & 4 & -4.10427 & 1.0000 & 12 & 6.243375 & 0.0000 & $3.57 E-03$ & MDNS_P & 4 & -4.1429 & 1.0000 & 12 & 6.264327 & 0.0002 & $3.19 \mathrm{E}-03$ \\
\hline MDNS_Lambda & 2 & -4.22478 & 1.0000 & 1 & -0.1801 & 1.0000 & $9.95 E-04$ & MDNS_Lambda & 2 & -4.26576 & 1.0000 & 1 & -0.09258 & 1.0000 & $9.94 \mathrm{E}-04$ \\
\hline MDNS_Macro & 7 & -1.16416 & 1.0000 & 6 & 1.681613 & 0.7066 & 4.70E-02 & MDNS_Macro & 7 & -1.1706 & 1.0000 & 6 & 1.663179 & 0.7216 & 4.07E-02 \\
\hline MDNS_MMacro & 8 & -0.27429 & 1.0000 & 9 & 2.206788 & 0.3194 & $7.46 \mathrm{E}-02$ & MDNS_MMacro & 8 & -0.16975 & 1.0000 & 9 & 2.157459 & 0.3586 & $6.70 \mathrm{E}-02$ \\
\hline MDNS_PMacro & 6 & -3.73568 & 1.0000 & 4 & 1.215098 & 0.9684 & $7.06 E-03$ & MDNS_PMacro & 6 & -3.66393 & 1.0000 & 4 & 1.190269 & 0.9706 & $7.06 \mathrm{E}-03$ \\
\hline MDNS_MMacroEnd & 5 & -4.06718 & 1.0000 & 11 & 2.597966 & 0.1552 & $8.18 \mathrm{E}-03$ & MDNS_MMacroEnd & 5 & -4.07694 & 1.0000 & 11 & 2.598044 & 0.1558 & 7.63E-03 \\
\hline MDNS_PMacroEnd & 9 & 0.11186 & 1.0000 & 5 & 1.330682 & 0.9276 & $9.21 \mathrm{E}-02$ & DNS_PMacroEnd & 9 & 0.235962 & 0.9992 & 5 & 1.328512 & 0.9252 & $8.71 \mathrm{E}-02$ \\
\hline MDNS_S & 11 & 1.438277 & 0.5462 & 10 & 2.213682 & 0.3158 & $2.08 E-01$ & MDNS_S & 11 & 1.392506 & 0.5790 & 10 & 2.210433 & 0.3272 & 1. $69 \mathrm{E}-01$ \\
\hline MDNS_SmediaMacro & 10 & 1.42435 & 0.5562 & 8 & 2.073964 & 0.4018 & $2.33 \mathrm{E}-01$ & MDNS_SmediaMacro & 10 & 1.388603 & 0.5834 & 7 & 2.015862 & 0.4508 & 2.03E-01 \\
\hline MDNS_Smacro & 12 & 1.647187 & 0.4184 & 7 & 2.024297 & 0.4346 & $3.42 E-01$ & MDNS_Smacro & 12 & 1.639983 & 0.4282 & 8 & 2.036546 & 0.4354 & $2.86 \mathrm{E}-01$ \\
\hline$p$-value: 0.4184 & & & & & & & & $p$-value: 0.4282 & & & & & & & \\
\hline & & Maturid & ade de 12 & neses & & & & & & Maturid & ade de 15 & neses & & & \\
\hline Modelos & Rank M & $v M$ & MCS M & Rank R & $\vee R$ & MCS R & Loss & Modelos & Rank M & $v M$ & MCS M & Rank R & $\vee R$ & MCS R & Loss \\
\hline MDNS & 2 & -4.31009 & 1.0000 & 2 & 0.241087 & 1.0000 & $9.74 \mathrm{E}-04$ & MDNS & 2 & -4.32633 & 1.0000 & 2 & 0.470781 & 1.0000 & $9.31 \mathrm{E}-04$ \\
\hline MDNS_M & 3 & -4.30837 & 1.0000 & 3 & 0.565048 & 1.0000 & $9.85 E-04$ & MDNS_M & 3 & -4.32403 & 1.0000 & 3 & 0.802889 & 0.9990 & $9.47 \mathrm{E}-04$ \\
\hline MDNS_P & 4 & -4.18553 & 1.0000 & 12 & 6.392127 & 0.0000 & $2.86 \mathrm{E}-03$ & MDNS_P & 4 & -4.2026 & 1.0000 & 12 & 6.504749 & 0.0000 & $2.57 \mathrm{E}-03$ \\
\hline MDNS_Lambda & 1 & -4.31067 & 1.0000 & 1 & -0.24109 & 1.0000 & $9.64 \mathrm{E}-04$ & MDNS_Lambda & 1 & -4.3276 & 1.0000 & 1 & -0.47078 & 1.0000 & $9.13 \mathrm{E}-04$ \\
\hline MDNS_Macro & 7 & -1.15223 & 1.0000 & 6 & 1.68805 & 0.6876 & 3.59E-02 & MDNS_Macro & 7 & -1.1197 & 1.0000 & 6 & 1.667211 & 0.7188 & $3.21 \mathrm{E}-02$ \\
\hline MDNS_MMacro & 8 & -0.0714 & 1.0000 & 9 & 2.127517 & 0.3674 & $6.12 \mathrm{E}-02$ & MDNS_MMacro & 8 & 0.026298 & 1.0000 & 9 & 2.114115 & 0.3826 & $5.65 E-02$ \\
\hline MDNS_PMacro & 6 & -3.62416 & 1.0000 & 4 & 1.19867 & 0.9724 & 7.13E-03 & MDNS_PM & 6 & -3.48086 & 1.0000 & 4 & 1.230819 & 0.9624 & $7.18 \mathrm{E}-03$ \\
\hline MDNS_MMacroEnd & 5 & -4.08191 & 1.0000 & 11 & 2.579318 & 0.1576 & $7.22 \mathrm{E}-03$ & MDNS_Mn & 5 & -4.0606 & 1.0000 & 11 & 2.603868 & 0.1530 & $6.89 \mathrm{E}-03$ \\
\hline MDNS_PM & 9 & 0.364459 & 0.9980 & 5 & 1.339658 & 0.9202 & $8.45 E-02$ & MDNS_PMacroEnd & 9 & 0.478285 & 0.9930 & 5 & 1.33955 & 0.9222 & $8.28 \mathrm{E}-02$ \\
\hline MDNS_S & 10 & 1.304105 & 0.6384 & 10 & 2.182474 & 0.3318 & $1.39 \mathrm{E}-01$ & MDNS_S & 10 & 1.230847 & 0.6848 & 10 & 2.151936 & 0.3606 & 1.16E-01 \\
\hline MDNS_SmediaMacro & 11 & 1.344664 & 0.6096 & 7 & 1.942909 & 0.4874 & $1.78 E-01$ & MDNS_SmediaMacro & 11 & 1.367869 & 0.5964 & 7 & 1.96325 & 0.4862 & $1.58 \mathrm{E}-01$ \\
\hline MDNS_Smacro & 12 & 1.620554 & 0.4272 & 8 & 2.039332 & 0.4224 & $2.41 \mathrm{E}-01$ & MDNS_Smacro & 12 & 1.6531 & 0.4134 & 8 & 2.108236 & 0.3862 & $2.05 \mathrm{E}-01$ \\
\hline p-value: 0.4272 & & & & & & & & p-value: 0.4134 & & & & & & & \\
\hline & & Maturid & ade de 18 & neses & & & & & & Maturid & ade de 21 & neses & & & \\
\hline Modelos & Rank M & $\vee M$ & MCS M & Rank $\mathrm{R}$ & $\vee R$ & MCS R & Loss & Modelos & Rank M & $\vee M$ & MCS M & Rank $\mathrm{R}$ & $v \mathrm{R}$ & MCS R & Loss \\
\hline MDNS & 2 & -4.34089 & 1.0000 & 2 & 0.720577 & 0.9996 & $8.84 \mathrm{E}-04$ & MDNS & 2 & -4.30676 & 1.0000 & 2 & 0.94568 & 0.9948 & $8.42 \mathrm{E}-04$ \\
\hline MDNS_M & 3 & -4.33808 & 1.0000 & 3 & 1.139398 & 0.9814 & $9.03 E-04$ & MDNS_M & 3 & -4.30353 & 1.0000 & 5 & 1.422562 & 0.8734 & $8.64 \mathrm{E}-04$ \\
\hline MDNS_P & 4 & -4.22042 & 1.0000 & 12 & 6.60028 & 0.0000 & $2.31 \mathrm{E}-03$ & MDNS_P & 4 & -4.18897 & 1.0000 & 12 & 6.693134 & 0.0000 & 2.09E-03 \\
\hline MDNS_Lambda & 1 & -4.34363 & 1.0000 & 1 & -0.72058 & 1.0000 & $8.58 \mathrm{E}-04$ & MDNS_Lambda & 1 & -4.31074 & 1.0000 & 1 & -0.94568 & 1.0000 & $8.09 \mathrm{E}-04$ \\
\hline MDNS_Macro & 7 & -1.05993 & 1.0000 & 6 & 1.648948 & 0.7226 & 2.91E-02 & $\mathrm{Ma}$ & 7 & -1.05236 & 1.0000 & 6 & 1734 & 0.6988 & $2.67 \mathrm{E}-02$ \\
\hline MDNS_MMacro & 8 & 0.119414 & 1.0000 & 9 & 2.105267 & 0.3912 & $5.28 \mathrm{E}-02$ & S_Mn & 8 & 0.209891 & 0.9996 & 9 & 3071 & 0.3880 & 4.98E-02 \\
\hline MDNS_PMacro & 6 & -3.37826 & 1.0000 & 4 & 1.219811 & 0.9660 & $7.18 \mathrm{E}-03$ & MDNS_PMacro & 6 & -3.21136 & 1.0000 & 3 & 1.235329 & 0.9562 & $7.16 \mathrm{E}-03$ \\
\hline MDNS_MMacroEnd & 5 & -4.06347 & 1.0000 & 11 & 2.529029 & 0.1902 & $6.62 \mathrm{E}-03$ & MDNS_MMacroEnd & 5 & -3.99021 & 1.0000 & 11 & 2.475862 & 0.2044 & $6.40 \mathrm{E}-03$ \\
\hline MDNS_PMacroEnd & 9 & 0.566006 & 0.9830 & 5 & 1.32785 & 0.9216 & $8.15 \mathrm{E}-02$ & MDNS_PMacroEnd & 9 & 0.647826 & 0.9704 & 4 & 1.331879 & 0.9152 & $8.06 \mathrm{E}-02$ \\
\hline MDNS_S & 10 & 1.195924 & 0.7114 & 10 & 2.215268 & 0.3226 & $9.67 E-02$ & DNS_S & 10 & 1.094335 & 0.7760 & 10 & 2.196843 & 0.3330 & $8.15 \mathrm{E}-02$ \\
\hline MDNS_SmediaMacro & 11 & 1.354773 & 0.6002 & 7 & 1.936157 & 0.5058 & $1.42 \mathrm{E}-01$ & MDNS_SmediaMacro & 11 & 1.300721 & 0.6426 & 7 & 1.860872 & 0.5608 & $1.28 \mathrm{E}-01$ \\
\hline MDNS_Smacro & 12 & 1.616331 & 0.4342 & 8 & 2.095652 & 0.3970 & $1.76 \mathrm{E}-01$ & MDNS_Smacro & 12 & 1.598765 & 0.4508 & 8 & 2.107762 & 0.3902 & $1.52 \mathrm{E}-01$ \\
\hline p-value: 0.4342 & & & & & & & & p-value: 0.4508 & & & & & & & \\
\hline & & Maturid & ade de 24 & ses & & & & & & Maturid & ade de 27 & eses & & & \\
\hline Modelos & Rank M & $\vee M$ & MCS M & Rank R & $\vee R$ & MCS R & Loss & Modelos & Rank M & $\vee M$ & MCS M & Rank $\mathrm{R}$ & $v R$ & MCS R & Loss \\
\hline MDNS & 2 & -4.2453 & 1.0000 & 2 & 1.181812 & 0.9734 & $8.03 E-04$ & MDNS & 2 & -4.17456 & 1.0000 & 4 & 1.410518 & 0.8888 & $7.71 \mathrm{E}-04$ \\
\hline MDNS_M & 3 & -4.24145 & 1.0000 & 6 & 1.711049 & 0.6722 & $8.27 \mathrm{E}-04$ & DNS_M & 3 & -4.17055 & 1.0000 & 7 & 1.998856 & 0.4606 & $7.96 \mathrm{E}-04$ \\
\hline MDNS_P & 4 & -4.13105 & 1.0000 & 12 & 6.68326 & 0.0000 & $1.90 E-03$ & MDNS_P & 4 & -4.06466 & 1.0000 & 12 & 6.761768 & 0.0000 & $1.75 \mathrm{E}-03$ \\
\hline MDNS_Lambda & 1 & -4.2503 & 1.0000 & 1 & -1.18181 & 1.0000 & 7.65E-04 & MDNS_Lambda & 1 & -4.18006 & 1.0000 & 1 & -1.24386 & 1.0000 & $7.28 \mathrm{E}-04$ \\
\hline MDNS_Macro & 7 & -0.9806 & 1.0000 & 5 & 1.673876 & 0.7000 & 2.47E-02 & NS_Macro & 7 & -0.93893 & 1.0000 & 5 & 1.67652 & 0.6994 & $2.32 \mathrm{E}-02$ \\
\hline MDNS_MMacro & 8 & 0.287217 & 0.9994 & 8 & 2.095544 & 0.3924 & 4.72E-02 & NS_MMacro & 8 & 0.355303 & 9980 & 8 & 2.052435 & 0.4208 & $4.51 \mathrm{E}-02$ \\
\hline MDNS_PMacro & 6 & -3.06763 & 1.0000 & 3 & 1.212274 & 0.9684 & 7.11E-03 & MDNS_PMacro & 6 & -2.92254 & 1.0000 & 2 & 1.243862 & 0.9604 & $7.05 \mathrm{E}-03$ \\
\hline MDNS_MMacroEnd & 5 & -3.89129 & 1.0000 & 11 & 2.41935 & 0.2254 & $6.22 \mathrm{E}-03$ & MDNS_MMacroEnd & 5 & -3.73571 & 1.0000 & 11 & 2.391545 & 0.2410 & $6.07 E-03$ \\
\hline MDNS_PMacroEnd & 9 & 0.723912 & 0.9494 & 4 & 1.342065 & 0.9192 & 7.99E-02 & MDNS_PMacroEnd & 9 & 0.760693 & 0.9380 & 3 & 1.308755 & 0.9338 & 7.93E-02 \\
\hline MDNS_S & 10 & 0.984241 & 0.8336 & 10 & 2.146978 & 0.3662 & $6.92 E-02$ & MDNS_S & 10 & 0.891047 & 0.8876 & 10 & 2.169456 & 0.3554 & $5.92 \mathrm{E}-02$ \\
\hline MDNS_SmediaMacro & 11 & 1.34383 & 0.6224 & 7 & 1.911463 & 0.5194 & 1.16E-01 & MDNS_SmediaMacro & 11 & 1.280241 & 0.6496 & 6 & 1.827014 & 0.5818 & $1.07 \mathrm{E}-01$ \\
\hline MDNS_Smacro & 12 & 1.568451 & 0.4790 & 9 & 2.106856 & 0.3856 & 1.32E-01 & MDNS_Smacro & 12 & 1.545888 & 0.4782 & 9 & 2.12309 & 0.3816 & 1.16E-01 \\
\hline
\end{tabular}




\begin{tabular}{|c|c|c|c|c|c|c|c|c|c|c|c|c|c|c|c|}
\hline \multicolumn{8}{|c|}{ Maturidade de 30 meses } & \multicolumn{8}{|c|}{ Maturidade de 33 meses } \\
\hline Modelos & Rank_M & $v \_M$ & MCS_M & Rank $R$ & $\vee R R$ & MCS R & Loss & Modelos & Rank_M & $v \_M$ & MCS_M & Rank $R$ & $V \_R$ & MCS_R & Loss \\
\hline MDNS & 2 & -4.11846 & 1.0000 & 4 & 1.580651 & 0.7680 & $7.47 \mathrm{E}-04$ & MDNS & 2 & -4.11468 & 1.0000 & 5 & 1.730355 & 0.6546 & $7.25 \mathrm{E}-04$ \\
\hline MDNS_M & 3 & -4.11401 & 1.0000 & 10 & 2.213259 & 0.3232 & 7.72E-04 & MDNS_M & 3 & -4.10991 & 1.0000 & 10 & 2.361493 & 0.2524 & 7.50E-04 \\
\hline MDNS_P & 4 & -4.01202 & 1.0000 & 12 & 6.705628 & 0.0000 & $1.62 \mathrm{E}-03$ & MDNS_P & 4 & -4.0115 & 1.0000 & 12 & 6.708961 & 0.0000 & $1.51 \mathrm{E}-03$ \\
\hline MDNS_Lambda & 1 & -4.12494 & 1.0000 & 1 & -1.258 & 1.0000 & $7.00 E-04$ & MDNS_Lambda & 1 & -4.12204 & 1.0000 & 1 & -1.23995 & 1.0000 & $6.75 E-04$ \\
\hline MDNS_Macro & 7 & -0.88662 & 1.0000 & 5 & 1.634919 & 0.7354 & 2.19E-02 & MDNS_Macro & 7 & -0.86764 & 1.0000 & 4 & 1.680061 & 0.6948 & $2.08 E-02$ \\
\hline MDNS_MMacro & 8 & 0.423528 & 0.9970 & 7 & 2.055263 & 0.4220 & $4.34 \mathrm{E}-02$ & MDNS_MMacro & 8 & 0.478832 & 0.9926 & 7 & 2.047769 & 0.4256 & $4.18 \mathrm{E}-02$ \\
\hline MDNS_PMacro & 6 & -2.81237 & 1.0000 & 2 & 1.257997 & 0.9512 & $6.97 \mathrm{E}-03$ & MDNS_PMacro & 6 & -2.73286 & 1.0000 & 2 & 1.239948 & 0.9542 & $6.89 \mathrm{E}-03$ \\
\hline MDNS_MMacroEnd & 5 & -3.63096 & 1.0000 & 11 & 2.427519 & 0.2190 & $5.95 \mathrm{E}-03$ & MDNS_MMacroEnd & 5 & -3.57861 & 1.0000 & 11 & 2.430135 & 0.2260 & $5.85 \mathrm{E}-03$ \\
\hline MDNS_PMacroEnd & 10 & 0.815378 & 0.9196 & 3 & 1.322808 & 0.9274 & $7.87 \mathrm{E}-02$ & MDNS_PMacroEnd & 10 & 0.863674 & 0.9032 & 3 & 1.335475 & 0.9186 & $7.83 E-02$ \\
\hline MDNS_S & 9 & 0.769847 & 0.9324 & 8 & 2.123294 & 0.3768 & $5.09 E-02$ & MDNS_S & 9 & 0.668725 & 0.9618 & 8 & 2.180227 & 0.3456 & 4.42E-02 \\
\hline MDNS_SmediaMacro & 11 & 1.282823 & 0.6600 & 6 & 1.821311 & 0.5972 & $9.87 E-02$ & MDNS_SmediaMacro & 11 & 1.26965 & 0.6560 & 6 & 1.811636 & 0.5924 & $9.17 E-02$ \\
\hline MDNS_Smacro & 12 & 1.547204 & 0.4864 & 9 & 2.184481 & 0.3390 & $1.03 \mathrm{E}-01$ & MDNS_Smacro & 12 & 1.529237 & 0.4854 & 9 & 2.211811 & 0.3280 & $9.16 \mathrm{E}-02$ \\
\hline \multicolumn{8}{|l|}{ p-value: 0.4864} & \multicolumn{8}{|l|}{ p-value: 0.4854} \\
\hline \multicolumn{8}{|c|}{ Maturidade de 36 meses } & \multicolumn{8}{|c|}{ Maturidade de 39 meses } \\
\hline Modelos & Rank_M & $v \_M$ & MCS_M & Rank_R & $V \_R$ & MCS_R & Loss & Modelos & Rank_M & V_M & MCS_M & Rank_R & $V \_R$ & MCS_R & Loss \\
\hline MDNS & 2 & -3.99145 & 1.0000 & 6 & 1.883784 & 0.5414 & $7.04 E-04$ & MDNS & 2 & -3.95343 & 1.0000 & 7 & 2.035326 & 0.4334 & $6.85 \mathrm{E}-04$ \\
\hline MDNS_M & 3 & -3.98662 & 1.0000 & 11 & 2.55512 & 0.1842 & $7.29 \mathrm{E}-04$ & MDNS_M & 3 & -3.9484 & 1.0000 & 11 & 2.706582 & 0.1330 & $7.10 \mathrm{E}-04$ \\
\hline MDNS_P & 4 & -3.89613 & 1.0000 & 12 & 6.763728 & 0.0000 & $1.41 \mathrm{E}-03$ & MDNS_P & 4 & -3.86209 & 1.0000 & 12 & 6.793078 & 0.0000 & $1.32 \mathrm{E}-03$ \\
\hline MDNS_Lambda & 1 & -3.99931 & 1.0000 & 1 & -1.25662 & 1.0000 & $6.52 \mathrm{E}-04$ & MDNS_Lambda & 1 & -3.96218 & 1.0000 & 1 & -1.2804 & 1.0000 & $6.32 \mathrm{E}-04$ \\
\hline MDNS_Macro & 7 & -0.78742 & 1.0000 & 4 & 1.637224 & 0.7264 & $1.99 \mathrm{E}-02$ & MDNS_Macro & 7 & -0.77285 & 1.0000 & 4 & 1.704397 & 0.6762 & $1.91 \mathrm{E}-02$ \\
\hline MDNS_MMacro & 8 & 0.525545 & 0.9880 & 7 & 2.037676 & 0.4302 & 4.05E-02 & MDNS_MMacro & 9 & 0.568616 & 0.9792 & 6 & 2.014531 & 0.4442 & $3.94 \mathrm{E}-02$ \\
\hline MDNS_PMacro & 6 & -2.596 & 1.0000 & 2 & 1.256623 & 0.9574 & $6.80 \mathrm{E}-03$ & MDNS_PMacro & 6 & -2.53651 & 1.0000 & 2 & 1.280403 & 0.9506 & $6.71 \mathrm{E}-03$ \\
\hline MDNS_MMacroEnd & 5 & -3.44485 & 1.0000 & 10 & 2.373604 & 0.2482 & $5.77 \mathrm{E}-03$ & MDNS_MMacroEnd & 5 & -3.33636 & 1.0000 & 10 & 2.377287 & 0.2476 & $5.69 \mathrm{E}-03$ \\
\hline MDNS_PMacroEnd & 10 & 0.892033 & 0.8880 & 3 & 1.323955 & 0.9282 & 7.79E-02 & MDNS_PMacroEnd & 10 & 0.92641 & 0.8674 & 3 & 1.331548 & 0.9236 & $7.76 E-02$ \\
\hline MDNS_S & 9 & 0.526887 & 0.9880 & 8 & 2.171133 & 0.3492 & 3.85E-02 & MDNS_S & 8 & 0.389221 & 0.9966 & 8 & 2.172444 & 0.3482 & 3.39E-02 \\
\hline MDNS_SmediaMacro & 11 & 1.232801 & 0.6862 & 5 & 1.761362 & 0.7264 & $8.57 E-02$ & MDNS_SmediaMacro & 11 & 1.254699 & 0.6590 & 5 & 1.789891 & 0.6762 & $8.05 E-02$ \\
\hline MDNS_Smacro & 12 & 1.499004 & 0.5112 & 9 & 2.218454 & 0.3226 & $8.22 \mathrm{E}-02$ & MDNS_Smacro & 12 & 1.478888 & 0.5136 & 9 & 2.268611 & 0.2960 & $7.42 \mathrm{E}-02$ \\
\hline \multicolumn{8}{|l|}{ p-value: 0.5112} & \multicolumn{8}{|l|}{ p-value: 0.5136} \\
\hline \multicolumn{8}{|c|}{ Maturidade de 48 meses } & \multicolumn{8}{|c|}{ Maturidade de 60 meses } \\
\hline Modelos & Rank_M & $\mathrm{v} \_\mathrm{M}$ & MCS_M & Rank_R & $V \_R$ & MCS_R & Loss & Modelos & Rank_M & V_M & MCS_M & Rank_R & $V \_R$ & MCS_R & Loss \\
\hline MDNS & 2 & -3.80218 & 1.0000 & 10 & 2.411813 & 0.2412 & $6.48 \mathrm{E}-04$ & MDNS & 2 & -3.58412 & 1.0000 & 10 & 2.732062 & 0.1348 & $6.16 \mathrm{E}-04$ \\
\hline MDNS_M & 3 & -3.79715 & 1.0000 & 11 & 3.240472 & 0.0474 & $6.71 \mathrm{E}-04$ & MDNS_M & 3 & -3.57937 & 1.0000 & 11 & 3.642885 & 0.0202 & $6.39 \mathrm{E}-04$ \\
\hline MDNS_P & 4 & -3.72242 & 1.0000 & 12 & 6.859352 & 0.0000 & $1.14 \mathrm{E}-03$ & MDNS_P & 4 & -3.51848 & 1.0000 & 12 & 7.083979 & 0.0000 & $9.88 \mathrm{E}-04$ \\
\hline MDNS_Lambda & 1 & -3.81192 & 1.0000 & 1 & -1.27296 & 1.0000 & $5.92 E-04$ & MDNS_Lambda & 1 & -3.595 & 1.0000 & 1 & -1.24622 & 1.0000 & $5.59 \mathrm{E}-04$ \\
\hline MDNS_Macro & 7 & -0.63742 & 1.0000 & 4 & 1.635058 & 0.7386 & $1.74 \mathrm{E}-02$ & MDNS_Macro & 8 & -0.53585 & 1.0000 & 4 & 1.701041 & 0.6848 & $1.61 \mathrm{E}-02$ \\
\hline MDNS_MMacro & 9 & 0.676447 & 0.9536 & 6 & 2.003316 & 0.4654 & $3.67 \mathrm{E}-02$ & MDNS_MMacro & 9 & 0.764877 & 0.9210 & 6 & 1.96248 & 0.4966 & $3.44 E-02$ \\
\hline MDNS_PMacro & 6 & -2.30239 & 1.0000 & 2 & 1.272959 & 0.9486 & $6.47 \mathrm{E}-03$ & MDNS_PMacro & 6 & -2.06364 & 1.0000 & 2 & 1.246216 & 0.9586 & $6.19 \mathrm{E}-03$ \\
\hline MDNS_MMacroEnd & 5 & -3.05382 & 1.0000 & 8 & 2.352684 & 0.2654 & $5.53 \mathrm{E}-03$ & MDNS_MMacroEnd & 5 & -2.75549 & 1.0000 & 8 & 2.289766 & 0.2980 & $5.39 \mathrm{E}-03$ \\
\hline MDNS_PMacroEnd & 10 & 0.994881 & 0.8120 & 3 & 1.336075 & 0.9200 & $7.68 \mathrm{E}-02$ & MDNS_PMacroEnd & 10 & 1.043834 & 0.7766 & 3 & 1.330083 & 0.9270 & $7.60 \mathrm{E}-02$ \\
\hline MDNS_S & 8 & -0.08408 & 1.0000 & 7 & 2.180476 & 0.4654 & $2.37 \mathrm{E}-02$ & MDNS_S & 7 & -0.75245 & 1.0000 & 7 & 2.152859 & 0.4966 & $1.58 \mathrm{E}-02$ \\
\hline MDNS_SmediaMacro & 11 & 1.218025 & 0.6790 & 5 & 1.759225 & 0.7386 & $6.86 \mathrm{E}-02$ & MDNS_SmediaMacro & 11 & 1.199246 & 0.6800 & 5 & 1.764696 & 0.6848 & $5.82 \mathrm{E}-02$ \\
\hline MDNS_Smacro & 12 & 1.412357 & 0.5592 & 9 & 2.398051 & 0.2654 & $5.67 \mathrm{E}-02$ & MDNS_Smacro & 12 & 1.263659 & 0.6408 & 9 & 2.507945 & 0.2980 & $4.26 E-02$ \\
\hline \multicolumn{8}{|l|}{ p-value: 0.5592} & \multirow{2}{*}{\multicolumn{8}{|c|}{ p-value: 0.6408}} \\
\hline & & Maturida & ade de 72 & neses & & & & & & & & & & & \\
\hline Modelos & Rank_M & V_M & MCS_M & Rank_R & $V \_R$ & MCS R $R$ & Loss & & & & & & & & \\
\hline MDNS & 2 & -3.43969 & 1.0000 & 10 & 3.056453 & 0.0660 & $5.94 \mathrm{E}-04$ & & & & & & & & \\
\hline MDNS_M & 3 & -3.4352 & 1.0000 & 11 & 4.022357 & 0.0100 & $6.14 \mathrm{E}-04$ & & & & & & & & \\
\hline MDNS_P & 4 & -3.38605 & 1.0000 & 12 & 6.994915 & 0.0000 & $8.84 \mathrm{E}-04$ & & & & & & & & \\
\hline MDNS_Lambda & 1 & -3.45073 & 1.0000 & 1 & -1.24556 & 1.0000 & $5.37 \mathrm{E}-04$ & & & & & & & & \\
\hline MDNS_Macro & 8 & -0.45626 & 1.0000 & 4 & 1.6965 & 0.6908 & $1.52 \mathrm{E}-02$ & & & & & & & & \\
\hline MDNS_MMacro & 9 & 0.844579 & 0.8776 & 6 & 2.023161 & 0.4478 & $3.28 \mathrm{E}-02$ & & & & & & & & \\
\hline MDNS_PMacro & 6 & -1.91145 & 1.0000 & 2 & 1.24556 & 0.9608 & $5.97 \mathrm{E}-03$ & & & & & & & & \\
\hline MDNS_MMacroEnd & 5 & -2.54087 & 1.0000 & 8 & 2.2913 & 0.2888 & $5.31 \mathrm{E}-03$ & & & & & & & & \\
\hline MDNS_PMacroEnd & 10 & 1.082758 & 0.7532 & 3 & 1.33472 & 0.9266 & $7.55 \mathrm{E}-02$ & & & & & & & & \\
\hline MDNS_S & 7 & -1.328 & 1.0000 & 7 & 2.148752 & 0.4478 & $1.12 \mathrm{E}-02$ & & & & & & & & \\
\hline MDNS_SmediaMacro & 12 & 1.143283 & 0.7112 & 5 & 1.719066 & 0.6908 & $5.14 \mathrm{E}-02$ & & & & & & & & \\
\hline MDNS_Smacro & 11 & 1.107202 & 0.7362 & 9 & 2.670547 & 0.2888 & $3.41 \mathrm{E}-02$ & & & & & & & & \\
\hline
\end{tabular}

*Modelos não reportandos nas tabelas foram eliminados pelo teste. 